\title{
GCU
}

Glasgow Caledonian

University

University for the Common Good

\section{What adherence measures should be used in trials of home-based rehabilitation interventions? A systematic review of the validity, reliability and acceptability of measures}

Frost, Rachael; Levati, Sara; McClurg, Doreen; Brady, Marian; Williams, Brian

Published in:

Archives of Physical Medicine and Rehabilitation

DOI:

10.1016/j.apmr.2016.08.482

Publication date:

2017

Link to publication in ResearchOnline

Citation for published version (Harvard):

Frost, R, Levati, S, McClurg, D, Brady, M \& Williams, B 2017, 'What adherence measures should be used in trials of home-based rehabilitation interventions? A systematic review of the validity, reliability and acceptability of measures', Archives of Physical Medicine and Rehabilitation, vol. 98, no. 6, e45, pp. 1241-1256.

https://doi.org/10.1016/j.apmr.2016.08.482

\section{General rights}

Copyright and moral rights for the publications made accessible in the public portal are retained by the authors and/or other copyright owners and it is a condition of accessing publications that users recognise and abide by the legal requirements associated with these rights.

Take down policy

If you believe that this document breaches copyright please view our takedown policy at https://edshare.gcu.ac.uk/id/eprint/5179 for details of how to contact us. 


\section{Accepted Manuscript}

What adherence measures should be used in trials of home-based rehabilitation interventions? A systematic review of the validity, reliability and acceptability of measures

Rachael Frost, PhD, Sara Levati, MSc, Doreen McClurg, PhD, Marian Brady, PhD, Brian Williams, $\mathrm{PhD}$

PII: S0003-9993(16)31135-2

DOI: 10.1016/j.apmr.2016.08.482

Reference: $\quad$ YAPMR 56685

To appear in: ARCHIVES OF PHYSICAL MEDICINE AND REHABILITATION

Received Date: 29 April 2016

Revised Date: 26 August 2016

Accepted Date: 31 August 2016

Please cite this article as: Frost R, Levati S, McClurg D, Brady M, Williams B, What adherence measures should be used in trials of home-based rehabilitation interventions? A systematic review of the validity, reliability and acceptability of measures, ARCHIVES OF PHYSICAL MEDICINE AND REHABILITATION (2016), doi: 10.1016/j.apmr.2016.08.482.

This is a PDF file of an unedited manuscript that has been accepted for publication. As a service to our customers we are providing this early version of the manuscript. The manuscript will undergo copyediting, typesetting, and review of the resulting proof before it is published in its final form. Please note that during the production process errors may be discovered which could affect the content, and all legal disclaimers that apply to the journal pertain. 
What adherence measures should be used in trials of home-based rehabilitation interventions? A systematic review of the validity, reliability and acceptability of measures

Rachael Frost, PhD, NMAHP Research Unit, Glasgow Caledonian University

Sara Levati, MSc, NMAHP Research Unit, Glasgow Caledonian University

Doreen McClurg, PhD NMAHP Research Unit, Glasgow Caledonian University

Marian Brady, PhD, NMAHP Research Unit, Glasgow Caledonian University

Brian Williams, PhD, School of Health \& Social Care, Edinburgh Napier University.

Corresponding author: Rachael Frost, University College London, Research Department of Primary Care and Population Health, Royal Free Campus, Rowland Hill Street, London, NW3 2PF, Tel: 0207 8302881, email: rachael.frost@ucl.ac.uk

Keywords: Patient compliance, reliability and validity, rehabilitation

Conflict of interest: The authors declare they have no conflicts of interest.

Acknowledgements: The authors would like to thank Dr Pauline Campbell, Heather

Strachan, Dr Katie Thomson and Dr Barbara Farquharson for undertaking secondary data extraction and quality assessment for this review.

Acknowledgement of financial support: Glasgow Caledonian University $\mathrm{PhD}$ studentship

PROSPERO ID: CRD42013004084. 
1 What adherence measures should be used in trials of home-based rehabilitation

2 interventions? A systematic review of the validity, reliability and acceptability of

3 measures

4

5 Keywords: Patient compliance, reliability validity, rehabilitation

6 Supplementary files: Medline search strategy, table of included studies, reference list of

$7 \quad$ included studies

8 
9

10 Abbreviations:

11 COSMIN COnsensus-based Standards for the selection of health Measurement

12

INstruments.

13 MPA measurement property assessment

14 OT

occupational therapist

15 PT

physiotherapist

$16 \mathrm{RCT}$

randomised controlled trial

17 SLT

speech and language therapist

18

19 


\section{Abstract}

22 Objective: To systematically review methods for measuring adherence used in home-based 23 rehabilitation trials, and evaluate their validity, reliability and acceptability.

Data sources: Phase 1: We searched CENTRAL, EED and HTA (Jan 2000-April 2013) to identify adherence measures used in randomised controlled trials of allied health professional home-based rehabilitation interventions. Phase 2: We searched Medline, Embase, CINAHL, AMED, PsycINFO, CENTRAL, ProQuest and Web of Science (inception-April 2015) for measurement property assessments (MPAs) for each measure.

Study selection: Studies assessing the validity, reliability or acceptability of adherence measures

Data extraction: Two reviewers independently extracted data on participant and measure characteristics, measurement properties evaluated, evaluation methods and outcome statistics and assessed study quality using the COSMIN checklist.

Data synthesis: Phase 1: We included 8 adherence measures ( $n=56$ trials). Phase 2: From 222 MPAs identified in 109 studies, 22 high quality MPAs were narratively synthesised. Low quality studies were used as supporting data. StepWatch Activity Monitor validly and acceptably measured short term step count adherence. The Problematic Experiences of Therapy Scale validly and reliably assessed adherence to vestibular rehabilitation exercises. Adherence diaries had moderately-high validity and acceptability across limited populations. The Borg 6-20 scale, Bassett \& Prapavessis' scale and the Yamax CW series had insufficient validity. Low quality evidence supported use of the Joint Protection Behaviour Assessment Polar A1 series heart monitors were considered acceptable by one study. 
43 Conclusions: Current rehabilitation adherence measures are limited. Some possess promising

$44 \quad$ validity and acceptability for certain parameters of adherence, situations and populations and

45 should be used in these situations. Rigorous evaluation of adherence measures in a broader

46 range of populations is needed.

47 Keywords: Patient compliance, reliability and validity, rehabilitation

48 PROSPERO ID: CRD42013004084.

49 
51 Adherence is the extent to which a person's behaviour coincides with agreed clinical

52 recommendations. ${ }^{1}$ Documenting participant adherence in clinical practice is necessary to monitor the patient's progress and help determine whether improvements (or lack of) is to be attributed to non/adherence or ineffectiveness of the prescribed therapy. Similarly, within clinical trials it is essential to measure adherence to answer the same question of attribution at a larger level, assess the impact of the intervention dose upon effectiveness, and to assist in identifying non-adherent patient subgroups. ${ }^{2}$ This is particularly vital within home-based rehabilitation interventions, where therapists expect greater independent patient engagement to prescribed therapeutic activities between formal therapy sessions. Prescribed home activities, e.g. home exercises, are an essential component within many allied health professional rehabilitation therapies, such as physiotherapy or occupational therapy. This reflects the increasing focus on functionally relevant rehabilitation, early supported discharge, ${ }^{3}$ maximising patient engagement with rehabilitation ${ }^{4}$ and self-management. ${ }^{5}$ Documenting adherence within clinical trials and practice can also provide an indication of the acceptability of an intervention to patients.

Given its vital role, the choice of adherence measurement method(s) should be guided by rigorous evidence of their respective measurement properties. Three prior systematic reviews have been undertaken in this area, focussing on: self-report adherence measures in homebased rehabilitation; ${ }^{6}$ patient or provider adherence questionnaires in physiotherapy ${ }^{7}$ and measures assessing adherence to non-pharmacological self-management in musculoskeletal conditions. ${ }^{8}$ All concluded that the available trials included largely self-developed

72 questionnaires that lacked sufficient evidence of measurement properties. ${ }^{6,8,9}$ A broader perspective was therefore required to encompass other methods in addition to questionnaires, based on methods currently used in clinical trials. Consequently, this review aimed 1) to 

identify adherence measurement methods used in rehabilitation clinical trials since 2000 and 2) to evaluate their validity, reliability and acceptability.

\section{Methods}

To address both review aims, we used a two-phase approach. In Phase 1 we identified recently used adherence measurement methods, and in Phase 2 we evaluated these methods according to the level of evidence for their measurement properties. The review protocol was registered in PROSPERO (ID CRD42013004084) and is reported according to PRISMA guidelines. $^{10}$

\section{Defining adherence}

Adherence is commonly defined in general terms, such as the World Health Organisation definition: "the extent to which a person's behaviour - taking medication, following a diet, and/or executing lifestyle changes, corresponds with agreed recommendations from a health care provider" (p.3). ${ }^{1}$ Whilst the breadth of this definition allows it to apply widely across many therapy types, it lacks the detail required to inform a useful operational definition for use in clinical practice or trials. Rehabilitation interventions are typically complex in nature and combine a number of parameters, to which patients may differentially adhere.

Rehabilitation prescriptions, similar to exercise or physical activity prescriptions, appear often to be characterised by four parameters in reviews or trials: frequency, duration, intensity and accuracy. ${ }^{11-14}$ For example, stroke patients seeking to improve mobility may be asked to carry out three balance exercises for five minutes each seven times a week. Despite adherence to the frequency of seven times per week, the patient may exercise for a shorter 
duration than recommended, may carry out just one of the three exercises or may carry out an exercise incorrectly.

Adherence was therefore operationalised within this review as the extent to which individuals undertake a prescribed behaviour accurately and at the agreed frequency, intensity and duration (see Figure 1). Measures assessing adherence to one or more of these parameters were included, in order to make recommendations across specific parameters and types of rehabilitation. "General adherence" was also included to identify any questionnaires based on the broader concept only.

\section{Phase 1 - Identifying currently used adherence measures}

Phase 1 aimed to collate a sample of adherence measurement methods used in home-based rehabilitation randomised controlled trials (RCTs). Rehabilitation is defined as the health strategy applied by professionals "that aims to enable people with health conditions experiencing or likely to experience disability achieve and maintain optimal functioning in interaction with the environment." (p.282). ${ }^{15}$ Physiotherapy (PT), occupational therapy (OT) and speech and language therapy (SLT) rehabilitation interventions were selected as allied health professionals whose therapies most commonly contain home-based components. We searched the Cochrane Central Register of Controlled Trials (CENTRAL), the NHS Economic Evaluation Database and the Health Technology Assessment database in April 2013 as a comprehensive source of rehabilitation clinical trials. We used the keywords adherence, compliance and rehabilitation (see Supplementary File 1). We limited the review to post-2000 as it was anticipated that relevant adherence measures developed before 2000 would carry forward into more recent usage. Hand searching was not used as adherence 
research is reported across multiple disciplines and research areas rather than within specific journals.

\section{Inclusion criteria:}

- $\quad$ Study design: RCTs, including protocols of RCTs

- $\quad$ Participants: adults with a health condition of any duration and severity

- Interventions: rehabilitation interventions including at least one of the following as part of a prescribed therapeutic regimen: modifications to the home environment or strategies to improve activities of daily living, home-based physical or language exercises or home-based interventions led by PTs, OTs or SLTs or an unspecified professional but the intervention met all other inclusion criteria; interventions to increase adherence to one of the above interventions.

- Comparators: any

- Outcomes: any method of measuring adherence to the concepts outlined above, including proxy measures, to the home-based component of the intervention.

- Studies carried out in countries where English is the primary language to ensure applicability to English-speaking populations.

\section{Exclusion criteria:}

Studies were excluded if they assessed the following: healthcare professional adherence to guidelines or study protocols; clinic- or hospital-based adherence only; group- or class-based adherence only; nutritional or pharmacological interventions only; primary prevention or screening initiatives; increasing physical activity in general rather than prescribed therapy. 
One reviewer screened titles, abstracts and full texts for relevant clinical trials, taking an inclusive approach and checking with a second reviewer (SL) in cases of uncertainty. Both reviewers (RF and SL) extracted data from included studies using a standardised data extraction form, regarding intervention characteristics; sample demographics; adherence measurement method used and component of adherence measured; adherence definition and outcome used; assessment location; completion rates; and references to relevant measurement property studies. In cases of disagreement, consensus was reached through discussion or consultation with a third reviewer (BW). Risk of bias was not assessed as we aimed to compile measurement methods rather than utilise the trials' findings. Titles of adherence measurement methods identified in Phase 1 contributed to Phase 2.

\section{Phase 2 - Evaluating the measurement properties of each method}

Within Phase 2 we aimed to evaluate the validity, reliability and acceptability of each named measurement method located in Phase 1, defined as:

i. Validity: whether an instrument measures what it intends to ${ }^{16}$, including:

a. Criterion validity: the closeness of a measure with the recognised gold standard or how well it predicts future outcomes. ${ }^{16}$

b. Construct validity: testing a hypothesised network of relationships and inferring the validity of the instrument from the results of these tests. ${ }^{16,17}$

c. Structural validity: the degree to which questionnaire scores reflect the dimensionality of the constructs measured. ${ }^{18}$

d. Face validity: the relevance and clarity of the measure at face value according to respondents or investigators' assessments. ${ }^{19,20}$ 
e. Content validity: systematic examination of the extent to which the instrument covers all elements requiring measurement in sufficient detail. ${ }^{19}$

f. Responsiveness to change: a measure's ability to detect change, ideally those that are clinically important. ${ }^{21}$

ii. Reliability: the extent to which a measure is free from random error. ${ }^{17}$

a. Test-retest reliability: reproducibility of a measure over a short period of time where the variable is not expected to change. ${ }^{20}$

b. Measurement error: the discrepancy between the observable concept measured and the actual underlying variable.

c. Inter-rater reliability: the agreement between two or more raters assessing the same population. ${ }^{20}$

d. Intra-rater reliability: the agreement between the same rater on the same subject on the same occasion ${ }^{22}$

e. Internal consistency: the homogeneity of scale items ${ }^{20}$

iii. Acceptability: the patient's willingness or ability to complete a measure, ${ }^{23}$ including data from any study type regarding wear time or rates (devices), completion rates, qualitative interviews, focus groups or think aloud studies and survey opinions or rating scales. ${ }^{24,25}$

Measurement properties were based on Classical Test Theory concepts with Item Response Theory (a questionnaire-specific theory that models the relationship between questionnaire items and the person's level of the construct ${ }^{26}$ ) MPAs include where relevant e.g. internal consistency, structural validity . Acceptability was considered a third key characteristic as adherence measures often require participants to wear or complete instruments more frequently than other outcome measures. 
191

192

193

194

195

196

197

198

199

200

201

202

203

204

205

206

207

208

209

210

211

212

Medline, CENTRAL, ProQuest Nursing \& Allied Health, EMBASE, CINAHL, AMED and

Web of Science Core Collection were searched initially from inception to April 2015 (see

Appendix 1 for Medline example of search terms). An earlier version of this review can be

found as a conference abstract. ${ }^{27}$ For each measure the title, with synonyms where applicable, was combined with acceptability search terms and Terwee et al's ${ }^{28}$ MPA study precise filter.

Subject headings were adapted for each database. Hand searching and consultation of topic experts were infeasible in such a diverse topic area and searching for ongoing MPAs was not possible as clinical trials registries are focussed on trials only.

Inclusion criteria:

- $\quad$ Participants: adults (healthy or clinical populations).

- Study types: studies assessing one or more MPAs outlined above in relation to the frequency, intensity, duration, accuracy or general adherence of an exercise or activity.

- $\quad$ Setting: laboratory and 'real-world' assessments.

- $\quad$ Adherence measure: the specific model or questionnaire type listed in Phase 1 only.

- Comparator: any comparator that could be classed or was described as a gold standard (criterion only) or measured a related aspect to the adherence component measured (construct only)

Exclusion Criteria: 
We excluded papers: not written in English; cross-cultural validity assessments, and therefore studies where the measure was used or administered in a language other than English; where the relevant measure was used to validate another measure; where the measure assessed symptoms, functional limitations or total energy expenditure rather than an adherence parameter; water-based activity; articles focussed on sports science applications rather than health science; conference abstracts (limited information) or reviews (relevant systematic review reference lists were screened).

Study screening was undertaken as per Phase 1 . Two reviewers (RF and either HS, BF, KT or PC) independently extracted data regarding: population, MPA type, sample size, activity, comparator(s) used, statistical methods, results and conclusions. Both independently assessed study quality using the COSMIN 4-point checklist ${ }^{29}$ and resolved disagreements through discussion. COSMIN scores measurement property studies as Poor, Fair, Good or Excellent based on their methodological features according to a least-score-counts system. Though this checklist has limited applicability to electronic measures as it was developed for patientreported outcome measures, it is the only comprehensive, well-developed checklist currently available for MPAs. We intended to synthesise studies of all quality; however, due to a large number of small, lower quality studies, the protocol was refined to include only Excellent or Good studies in the main narrative synthesis. This ensured that conclusions were based on high quality evidence, whilst Poor or Fair rated studies were used in a sensitivity analysis to see if they confirmed, refuted or extended the higher quality study findings. Study authors were contacted where possible in the event of missing data.

Studies were tabulated according to measurement method, MPA type and parameters of adherence the method was validated for. We aggregated studies using the Centre for Reviews and Dissemination's narrative synthesis approach. ${ }^{30}$ Whilst statistics such as limits of agreement are in the original units and so have a more straightforward interpretation ${ }^{31}$, there 
is little consensus as to the interpretation of statistics which give a value between 0 and 1 (e.g. correlations, kappa, alpha). As we did not plan to conduct meta-analyses, we grouped values to assist comparisons. A minimum acceptable value was not used as we accepted that this would differ according to the measurement needs of different situations. High values are generally considered to be $>0.70$, preferably $>0.80^{32-35}$, therefore we used the following cut offs, based on commonly used rules of thumb, to classify correlations, alpha, kappa and percentage wear/completion rates: ${ }^{36}$

- Poor: 0.00-0.19, 0-19\%

- $\quad$ Fair: 0.20-0.39, 20-39\%

- Moderate: $0.40-0.59,40-59 \%$

- Good: $0.60-0.79,60-79 \%$

- $\quad$ Excellent: $>0.80,80-100 \%$

Other acceptability results were descriptively summarised due to the heterogeneity of the methods used (e.g. qualitative interviews, completion rates).

\section{Results}

Figure 2 shows the flow of studies throughout Phase 1 and 2.

[Figure 2 about here]

\section{Phase 1- Identifying currently used adherence measures}

Within Phase 1, 56 datasets of 59 full texts were included out of 1174 initial references and 209 full texts. Twenty eight were checked with a second reviewer (SL). Interventions were classified as discipline-specific as per the professional described in the text, and were largely 
physiotherapy-based $(n=36)$. Musculoskeletal conditions $(n=27)$ were most commonly treated in the included trials. Thirty five single and 21 combinations of adherence measurement methods were identified (see Table 1). Frequency adherence was most commonly measured $(n=44)$, followed by duration $(n=15)$, intensity $(n=14)$ and general adherence $(n=12)$.

Accuracy was only measured in four RCTs. Adherence diaries were assumed to measure frequency only if no further details were given. Common adherence outcomes used were average percentage sessions $(n=17)$, average number of sessions $(n=14)$ and percentage achieving minimum adherence levels $(n=10)$.

Seven named methods were identified. One questionnaire used in two studies ${ }^{37,38}$ was not named but the RCT reports contained measurement property information. This scale, termed Bassett \& Prapavessis' scale after the study authors, was included in Phase 2 but as Phase 2 search strategies incorporated measure titles further measurement property searches were not feasible for this scale. "Cited by" functions did not reveal further studies. We therefore evaluated the following eight methods in Phase 2, which are summarised in Table 2 along with their MPAs.

[Table 2 about here]

\section{Phase 2 - Evaluating the measurement properties of each method}

The initial and updated results were combined, de-duplicated and rescreened as necessary. Out of 6926 hits across both reviews, 869 full texts were screened and 109 studies including 222 MPAs were included (18 articles checked by a second reviewer). After applying COSMIN criteria ${ }^{29}, 22$ Excellent or Good MPAs were included in the synthesis, 153 low quality studies were used as supporting data and 47 acceptability studies were evaluated. 
284

285

286

287

288

289

290

291

292

293

294

295

296

297

298

299

300

301

302

303

304

305

306

307

These are summarised alongside a description of each measure in Table 2, with details of each study tabulated in Supplementary File 2. Three MPAs are awaiting further information. $^{39-41}$

To summarise, the evidence for most measures was limited. The StepWatch Activity Monitor appeared to be the most valid measure of adhering to a daily step count, but the evidence base consisted largely of short-term laboratory studies, was inconsistent across populations and lacked predictive validity (see Table 2). It appeared to be reliable and acceptable to wear for one week and up to 28 days. Adherence diaries had good to excellent criterion validity in the limited populations they were validated in, but lacked predictive validity of functional outcomes. Evidence for their reliability was scarce, but acceptability ranged from moderate to excellent (50-100\% return rates). Regarding questionnaires, the Problematic Experiences of Therapy Scale had greater validity, reliability and acceptability for assessing general adherence than Bassett \& Prapavessis' scale, though both had limited MPAs in single populations. The Borg 6-20 scale and CW series pedometers had inadequate validity, though these measures appeared to be reliable. The Joint Protection Behaviour Assessment had low quality supporting data for validity and reliability, whilst the Polar A1 heart rate monitor series had good acceptability in healthy adults but no other validity or reliability assessments. Sensitivity analyses largely confirmed the findings in broader patient populations and contributed reliability data.

\section{Discussion}

In this systematic review we found that adherence diaries were the most commonly used measures, usually for assessing adherence to how frequently a home-based behaviour was carried out. Self-developed questionnaires were also common, whilst most named methods 
were sparsely used. The eight named methods identified had limited evidence, with suggestions that the StepWatch Activity Monitor and adherence diaries may be valid and acceptable within certain populations. Other methods lacked measurement properties or were assessed only in limited populations.

\section{Strengths}

As found in previous reviews of adherence to physiotherapy, rehabilitation and selfmanagement adherence systematic reviews, we found an abundance of self-developed questionnaires and diaries. ${ }^{6,8,9}$ However, these reviews found little evidence of measurement properties for any of the included measures. The larger volume found in this review is likely to arise from including electronic measures and aggregating diaries (often considered as a single type of measure).

In order to confirm the relevance of the measures considered in Phase 2 above we updated our Phase 1 search in August 2016. Out of the 41 new studies identified in the update adherence diaries (34 studies), Step Watch Activity Monitors (2 studies) Yamax CW-701, Borg 6-20 scale and the Problematic Experiences of Therapy Scale (each 1 study) continued to be reported. Additional non-named methods were also reported (as in our Phase 1 review) including sensors in hardware or software $(n=5)$, self-developed questionnaires $(n=6)$, telephone interviews $(n=6)$, carer reports $(n=1)$ and an accuracy checklist developed for the study $(n=1)$. Some newly emerging measures were also reported within isolated studies including the Exercise Adherence Rating Scale, the Omron HJ-720ITC Pocket pedometer, the Borg CR-10 scale, the Accusplit pedometer, and the Adherence Assessment. These new methods remain avenues for further review alongside measures developed in non-English languages, in trials not indexed in CENTRAL or not yet employed in a rehabilitation clinical trial. 
332 To our best knowledge this review is the first to provide a rigorous assessment and summary of multiple types of adherence measures across a broad range of interventions, participants and professionals. In particular, previous reviews have neglected to evaluate the acceptability of each measure, which remains a vital part of adherence measurement, particularly when measures are worn or completed on a daily basis. Comparison across electronic, provider report and self-report methods, whilst complex, is vital for decision making and so this review has greater utility than one of a single measure or type of measure. Further strengths include the two-phase approach which ensured that relevant measures were assessed and the use of an explicit conceptual underpinning often absent in adherence measurement. We searched for a wide variety of measurement properties and two reviewers independently assessed study quality using the COSMIN checklist. Only one main protocol refinement occurred, which was to include only high quality studies, but this was deemed reasonable as it allowed recommendations to be made on the basis of the most rigorous evidence.

\section{Limitations}

Within Phase 2, some relevant measurement property assessments may not have been located due to inadequate definition, classification and reporting of these studies. Common limitations in the evidence base located included small sample sizes and suboptimal statistics in validity and reliability assessments. Only a small number of included studies were of high quality. Most were of Fair or Poor quality and used only small sample sizes. A large majority of the StepWatch studies were carried out in a lab, which limits generalisability to use in a home-based situation where a wider range of activity is likely to be recorded. Whilst laboratory environments lessen the clinical applicability of these studies, they were included as they provided some validity information and for some tools (e.g. the StepWatch Activity Monitor) assessing criterion validity outside of a laboratory is challenging. 
356

357

358

359

360

361

362

363

364

365

366

367

368

369

370

371

372

373

374

375

376

377

378

Other methods were tested in only limited populations e.g. the Borg scale was usually

validated for activities in healthy adults, despite its increasingly common usage in

rehabilitation. Diaries lacked reliability assessments, whilst all measures had a paucity of

reliability, responsiveness to change and predictive validity studies. Acceptability was rarely

formally assessed, despite wear and completion being important components of electronic

devices such as activity monitors or diaries. Defining adequate comparators was also

problematic as some included methods were used to validate others. ${ }^{42}$ Gold standards were

unavailable for some types of rehabilitation activity or for assessing adherence to behaviour accuracy.

\section{Implications for clinical practice}

When selecting adherence measures for use in clinical trials or clinical practice, conceptual adherence definitions need to be utilised. This permits a measure to be selected according to the level of rigorous evidence of measurement properties available for the relevant components. The main recommendations for using adherence measures in clinical trials and practice are summarised in Table 3. Most measures were validated in specific participant populations and prior to using a measure, clinicians should check it is validated for that population. Consequently our findings are likely to have the greatest relevance to physiotherapy and exercise-based interventions, as this was where most measures were used and evaluated, though some measures (e.g. adherence diaries) were used across all intervention types.

[Table 3 about here]

\section{Implications for future research}


Further well-designed, adequately powered studies, particularly reliability studies, evaluating a measure in therapeutic situations are required to inform future adherence measure selection. Formal qualitative evaluations by service users are required to further assess acceptability studies and better reporting of quantitative acceptability data. Identifying the most suitable measures for different populations will optimise their use in trials and clinical practice. Furthermore, this review showed that reviewing existing electronic measures (e.g. pedometers) warrants further investigation to determine their validity and acceptability for measuring adherence. The development of new questionnaires based upon a thorough adherence conceptualisation that takes accuracy or intensity into account may also be valuable. However current methods also offer potential for development and testing. This should be prioritised to avoid the multitude of self-developed questionnaires that are not comparable, as identified in the first phase of this review. Utilising adherence measures in RCTs presents further opportunities to collect feasibility, acceptability and MPA data regarding adherence measures. These should be reported clearly or separately to enable location of this data in future reviews.

\section{Conclusion}

Currently, there is no gold standard of adherence measurement for home-based therapies.

Methods included in this review are limited by the quality of evidence of their measurement properties or their limited applicability across interventions. However, in light of the available evidence, StepWatch Activity Monitors are likely to be valid and acceptable to assess adherence to walking interventions, adherence diaries can approximate adherence to intervention frequency and duration and the Problematic Experiences of Therapy Scale can validly and reliably assess general adherence across vestibular rehabilitation populations. 
403 Further study into which measures are most suitable for intervention parameters and patient 404 populations and clearer reporting is required.

405

406

407 
408

Tables

409 1. Summary of Phase 1 measurement types and adherence components measured

410 2. Summary of each included measure and its measurement properties

411 3. Implications for adherence measures identified in this review

$412 \quad$ Figures

413 1. Conceptual definition of adherence within this review

4142 . Flow of studies throughout the review

415

416

417 


\section{References}

419 1. World Health Organization. Adherence to long-term therapies: evidence for action. Geneva: 2003.

2. Kehoe SH, Chheda PS, Sahariah S, Baird J, Fall CHD. Reporting of participant compliance in randomized controlled trials of nutrition supplements during pregnancy. Matern. Child Nutr. 2009;5:97-103.

3. National Institute for Health and Care Excellence. Stroke rehabilitation: Long-term rehabilitation after stroke. Manchester:

4. National Institute for Health and Care Excellence. MI - secondary prevention: Secondary prevention in primary and secondary care for patients following a myocardial infarction.

5. Long-term Conditions Alliance Scotland. "Gaun Yersel!": The Self Management Strategy for Long Term Conditions in Scotland. Glasgow: 2008.

6. Bollen JC, Dean SG, Siegert RJ, Howe TE, Goodwin V. A systematic review of measures of self-reported adherence to unsupervised home-based rehabilitation exercise programmes, and their psychometric properties. BMJ Open. 2014;4:e005044.

7. Holden M, Haywood K, Potia T, Gee M, McLean S. Recommendations for exercise adherence measures in musculoskeletal settings: a systematic review and consensus meeting (protocol). Syst. Rev. 2014;3:10.

437 8. Hall AM, Kamper SJ, Hernon M, Lonsdale C, Hurley DA, Hughes K, et al. Measurement tools for adherence to non-pharmacological self-management treatment for chronic musculoskeletal conditions: a systematic review. Arch. Phys. Med. Rehabil. 2015;96:552-62. 
adherence measures - why we need to start again. Findings of a systematic review and consensus workshop. Physiotherapy. 2015;101:e981-2.

10. Moher D, Liberati A, Tetzlaff J, Altman DG, Grp P. Preferred Reporting Items for Systematic Reviews and Meta-Analyses: The PRISMA Statement (Reprinted from Annals of Internal Medicine). Phys. Ther. 2009;89:873-80.

11. Page SJ, Schmid A, Harris JE. Optimizing terminology for stroke motor rehabilitation: recommendations from the American Congress of Rehabilitation Medicine Stroke Movement Interventions Subcommittee. Arch. Phys. Med. Rehabil. 2012;93:1395-9.

12. Pollock M. Prescribing Exercise for Fitness and Adherence. In: Exercise Adherence: Its Impact on Public Health. Champaign: Human Kinetics; 1988. p. 259-77.

13. Simek EM, McPhate L, Haines TP. Adherence to and efficacy of home exercise programs to prevent falls: A systematic review and meta-analysis of the impact of exercise program characteristics. Prev. Med. (Baltim). 2012;55:262-75.

14. Smith J, Lewis J, Prichard D. Physiotherapy exercise programmes: Are instructional exercise sheets effective? Physiother. Theory Pract. 2005;21:93-102.

15. Stucki G, Cieza A, Melvin J. The International Classification of Functioning, Disability and Health (ICF): a unifying model for the conceptual description of the rehabilitation strategy. J. Rehabil. Med. 2007;39:279-85.

16. Streiner DL, Norman G. Health Measurement Scales: A practical guide to their development and use. 3rd ed. Oxford: Oxford University Press; 2003.

17. Carmines EG, Zeller RA. Reliability and Validity Assessment. London: Sage Publications; 1979.

18. Mokkink L, Terwee C, Patrick D, Alonso J, Stratford P, Knol D, et al. COSMIN checklist manual. 2012; 
19. Vitolins M, Rand C, Rapp S, Ribisl P, Sevick M. Measuring adherence to behavioral and medical interventions. Control. Clin. Trials. 2000;21:188S - 94S.

20. Bowling A. Techniques of questionnaire design. In: Bowling A, Ebrahim S, editors. Handbook of Health Research Methods. Maidenhead: Open University Press; 2005. p. $394-427$.

21. Guyatt G, Walter S, Norman G. Measuring Change Over Time: Assessing the usefulness of evaluative instruments. J. Chronic Dis. 1987;40:171-8.

22. Mokkink L, Terwee C, Patrick D, Alonso J, Stratford P, Knol D, et al. The COSMIN checklist for assessing the methodological quality of studies on measurement properties of health status measurement instruments: an international Delphi study. Qual. Life Res. 2010;19:539-49.

23. Haywood KL, Hargreaves J, White R, Lamb SE. Reviewing measures of outcome: reliability of data extraction. J. Eval. Clin. Pract. 2004;10:329-37.

24. De Bleser L, De Geest S, Vincke B, Ruppar T, Vanhaecke J, Dobbels F. How to test electronic adherence monitoring devices for use in daily life: a conceptual framework. Comput. Inform. Nurs. 2011;29:489-95.

25. Haywood KL, Staniszewska S, Chapman S. Quality and acceptability of patientreported outcome measures used in chronic fatigue syndrome/myalgic encephalomyelitis (CFS/ME): a systematic review. Qual. Life Res. 2012;21:35-52.

26. Edelen MO, Reeve BB. Applying item response theory (IRT) modeling to questionnaire development, evaluation, and refinement. Qual. Life Res. 2007;16 Suppl $1: 5-18$.

27. Frost R, Brady M, McClurg D, Williams B. A systematic review of adherence measurement methods currently used in randomised controlled trials of home-based 
rehabilitation interventions. Clin. Rehabil. 2015;29:396-7.

28. Terwee CB, Jansma EP, Riphagen II, De Vet HCW. Development of a methodological PubMed search filter for finding studies on measurement properties of measurement instruments. Qual. Life Res. 2009;18:1115-23.

29. COSMIN. COSMIN checklist with 4-point scale [Internet]. 2011;Available from: http://www.cosmin.nl/images/upload/files/COSMIN checklist with 4-point scale 22 juni 2011.pdf

30. Centre for Reviews and Dissemination. Systematic reviews: CRD's guidance for undertaking reviews in health care. York: University of York; 2008.

31. de Vet HCW, Terwee CB, Knol DL, Bouter LM. When to use agreement versus reliability measures. J. Clin. Epidemiol. 2006;59:1033-9.

32. Kottner J, Audigé L, Brorson S, Donner A, Gajewski BJ, Hróbjartsson A, et al. Guidelines for Reporting Reliability and Agreement Studies (GRRAS) were proposed. J. Clin. Epidemiol. 2011;64:96-106.

33. Terwee C, Mokkink L. Qualitative Attributes and Measurement Properties of Physical Activity Questionnaires. Sport. Med. 2010;40:525-37.

34. Fitzpatrick R, Davey C, Buxton M, Jones D. Evaluating patient-based outcome measures for use in clinical trials. Health Technol. Assess. (Rockv). 1998;2:14.

35. Cicchetti D V. The precision of reliability and validity estimates re-visited: distinguishing between clinical and statistical significance of sample size requirements. J. Clin. Exp. Neuropsychol. 2001;23:695-700.

36. Viera AJ, Garrett JM. Understanding interobserver agreement: The kappa statistic. Fam. Med. 2005;37:360-3. 
37. Bassett SF, Prapavessis H. A test of an adherence-enhancing adjunct to physiotherapy steeped in the protection motivation theory. Physiother. Theory Pract. 2011;27:36072.

38. Bassett SF, Prapavessis H. Home-based physical therapy intervention with adherenceenhancing strategies versus clinic-based management for patients with ankle sprains. Phys. Ther. 2007;87:1132-43.

39. Barak S. Habitual ambulatory activity measurement post-stroke. Diss. Abstr. Int. Sect. B Sci. Eng. 2009;

40. Bergman RJ, Bassett Jr DR, Muthukrishnan S, Klein DA. Validity of 2 devices for measuring steps taken by older adults in assisted-living facilities. J. Phys. Act. Health. 2008;5 Suppl 1:S166-75.

41. Wajciechowski J, Gayle R, Andrew R, Dintiman G. The accuracy of radio telemetry heart rate monitoring during exercise. Clin. Kinesiol. 1991;45:9-12.

42. Franklin PD, McLaughlin J, Boisvert CB, Li W, Ayers DC. Pilot study of methods to document quantity and variation of independent patient exercise and activity after total knee arthroplasty. J. Arthroplasty. 2006;21:157-63. 
Table 1. Number of measures found in Phase 1, by type and adherence parameter measured

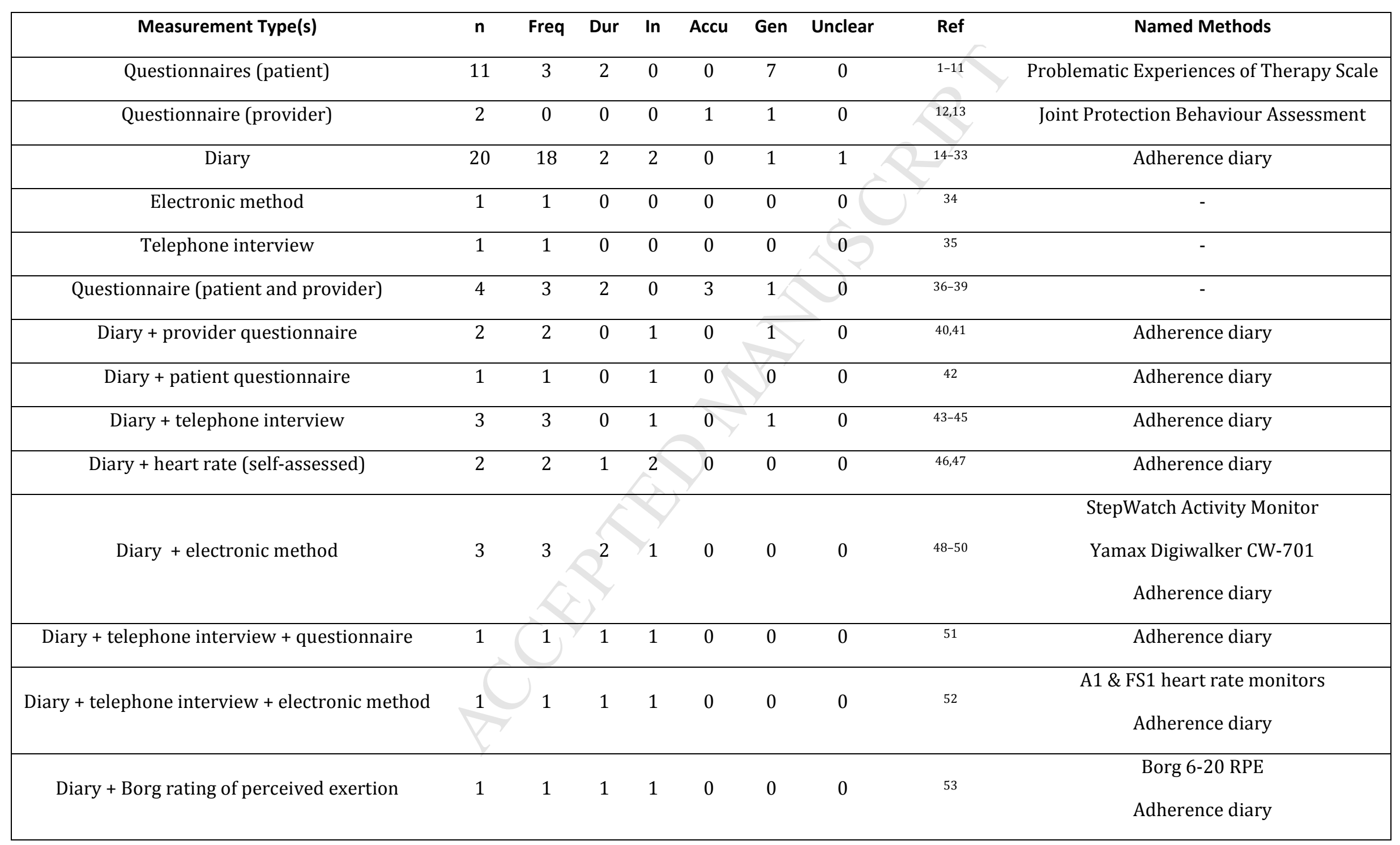




\begin{tabular}{|ccccccccccccc}
\hline $\begin{array}{c}\text { Diary + Borg rating of perceived exertion + self- } \\
\text { assessed heart rate }\end{array}$ & 2 & 2 & 2 & 2 & 0 & 0 & 0 & 54,55 & $\begin{array}{c}\text { Borg 6-20 RPE } \\
\text { Adherence diary }\end{array}$ \\
\hline $\begin{array}{c}\text { Diary + telephone interview + Borg rating of } \\
\text { perceived exertion + self-assessed heart rate }\end{array}$ & 1 & 1 & 1 & 1 & 0 & 0 & 0 & Borg 6-20 RPE \\
Adherence diary
\end{tabular}

Key: n=number of trials containing this measure; Freq=frequency, Dur=duration, In=Intensity, Accu=accuracy, Gen=general adherence, Ref=reference. The reference list for included studies can be found in Supplementary File 3. 


\section{Table 2. Summary of each included measure and its measurement properties}

\begin{tabular}{|c|c|c|c|c|}
\hline Measure & Description & Validity & Reliability & Acceptability \\
\hline \multirow[t]{17}{*}{ StepWatch } & Research-grade ankle-worn & High quality studies $(n=5)$ : Small percentage error and & High quality studies $(n=0)$ & $\mathrm{N}=24$. Highly acceptable across \\
\hline & activity monitor. ${ }^{57}$ Described as & mean bias and high percentage accuracy compared to & & populations for $1-28$ days' wear \\
\hline & a pedometer, accelerometer or & direct observation for measuring step counts in healthy & & (most commonly worn for 1 week), \\
\hline & activity monitor as the internal & populations, individuals with COPD and individuals & & including persons with MS, TKA, \\
\hline & mechanisms have not been & with MS in laboratory settings. ${ }^{59-61}$ Fair predictive & & neurological conditions, sarcoma, \\
\hline & disclosed. ${ }^{58}$ & validity in persons with intermittent claudication for & & lower limb prosthesis, knee OA and \\
\hline & & changes in Peak Walking Time. ${ }^{48}$ & & older, sedentary and obese adults. \\
\hline & & & & In most studies $>90 \%$ patients \\
\hline & & Low quality studies $(n=20)$ : Small mean bias and percentage & Low quality studies $(n=14)$ : Excellent & \\
\hline & & error and high percentage accuracy were confirmed in older & test-retest reliability for step counts & complied with SAM wear, but this \\
\hline & & adults, healthy volunteers and individuals with COPD, & same day to 3 weeks apart in the lab & was variable in stroke survivors and \\
\hline & & neurological conditions and mobility limitations). ${ }^{82-98}$ Lower & or home/ community in persons who & lower in persons with dementia, \\
\hline & & validity in persons with dementia, ${ }^{77}$ cycling activity, ${ }^{86}$ & are healthy or with neurological & persons with intermittent \\
\hline & & outdoor walking on a paretic $\operatorname{limb}^{95}$ and when attached to a & conditions (wider LOA in & claudication and healthy adults. ${ }^{48,62-}$ \\
\hline & & cane. ${ }^{98}$ Moderate construct validity for activity intensity & community). ${ }^{62,72,75,77,85,94,97,99-101}$ & ${ }^{77}$ Most wore the SAM for $>6$ out of \\
\hline & & compared to a diary. ${ }^{85}$ & Excellent inter-rater reliability in & 7 days per week ${ }^{63,66,78-80}$ and $>11$ \\
\hline & & & healthy adults. ${ }^{87}$ & hours per day. ${ }^{69,80,81}$ \\
\hline
\end{tabular}




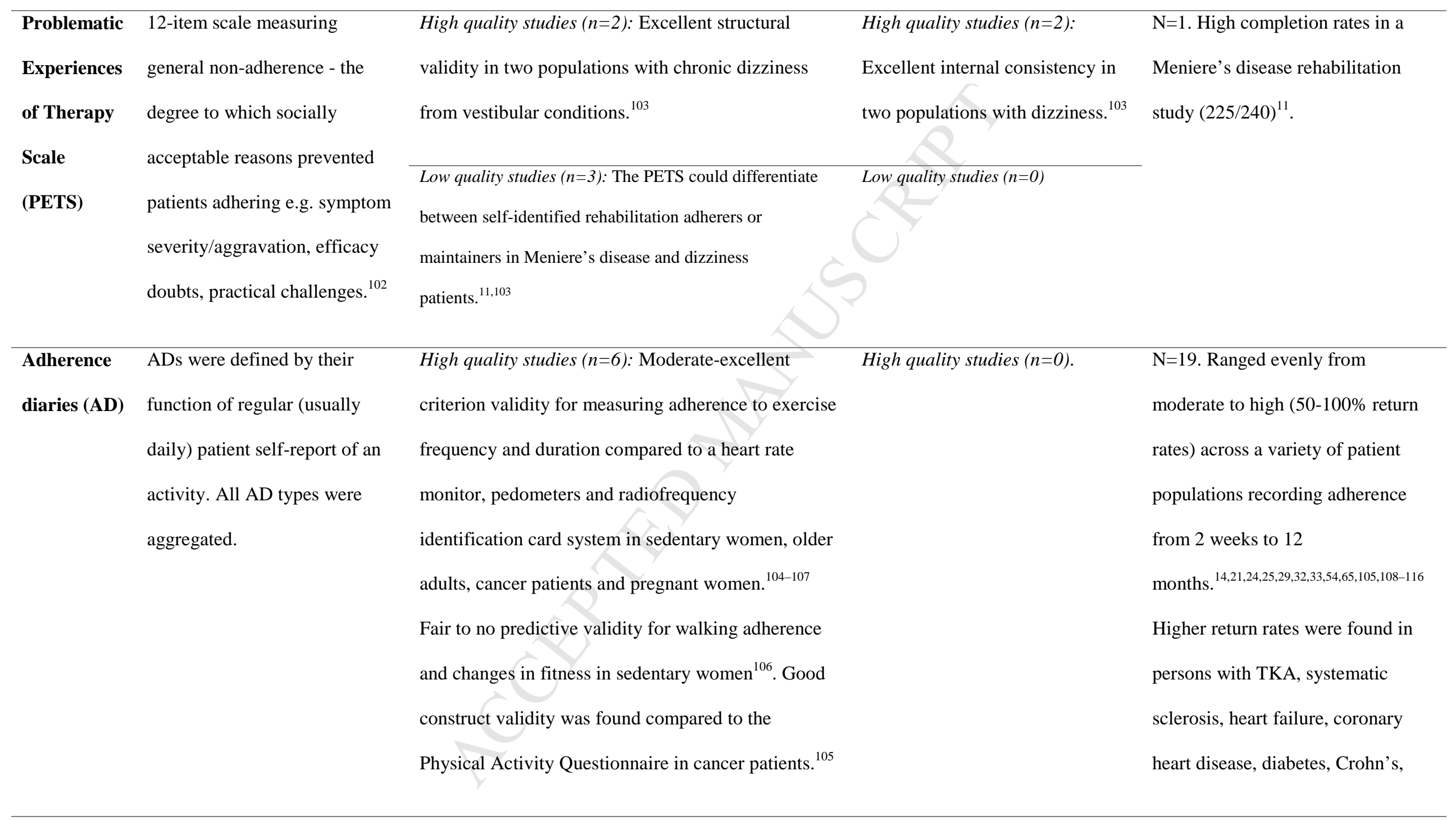


Low quality studies ( $n=19)$ : Good to excellent criterion

validity compared to a range of objective comparators in

varied populations (older adults, knee arthroplasty patients,

individuals with pain conditions, brain injury and

SLE) ${ }^{65,117-122}$ Low to moderate predictive validity for

functional outcome measures in individuals with COPD,

sedentary women, individuals with radial fracture, total

knee arthroplasty patients and patients with implantable

cardiac defibrillators. ${ }^{65,109,123-125}$. Moderate to good

construct validity for exercise-related constructs in

sedentary women and healthy adults, but lower validity for

behavioural constructs in persons with Huntingdon's

disease and sedentary women,. ${ }^{125-128}$ Adherence predicted

maintenance in sedentary women. ${ }^{129}$ Diaries were

responsive to short-term adherence changes in pulmonary

rehabilitation. ${ }^{130}$

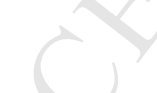

\section{Low quality studies $(n=1)$ : Good test-}

retest reliability in pregnant

women. ${ }^{104}$

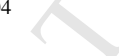

\section{(}
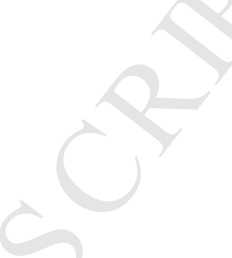


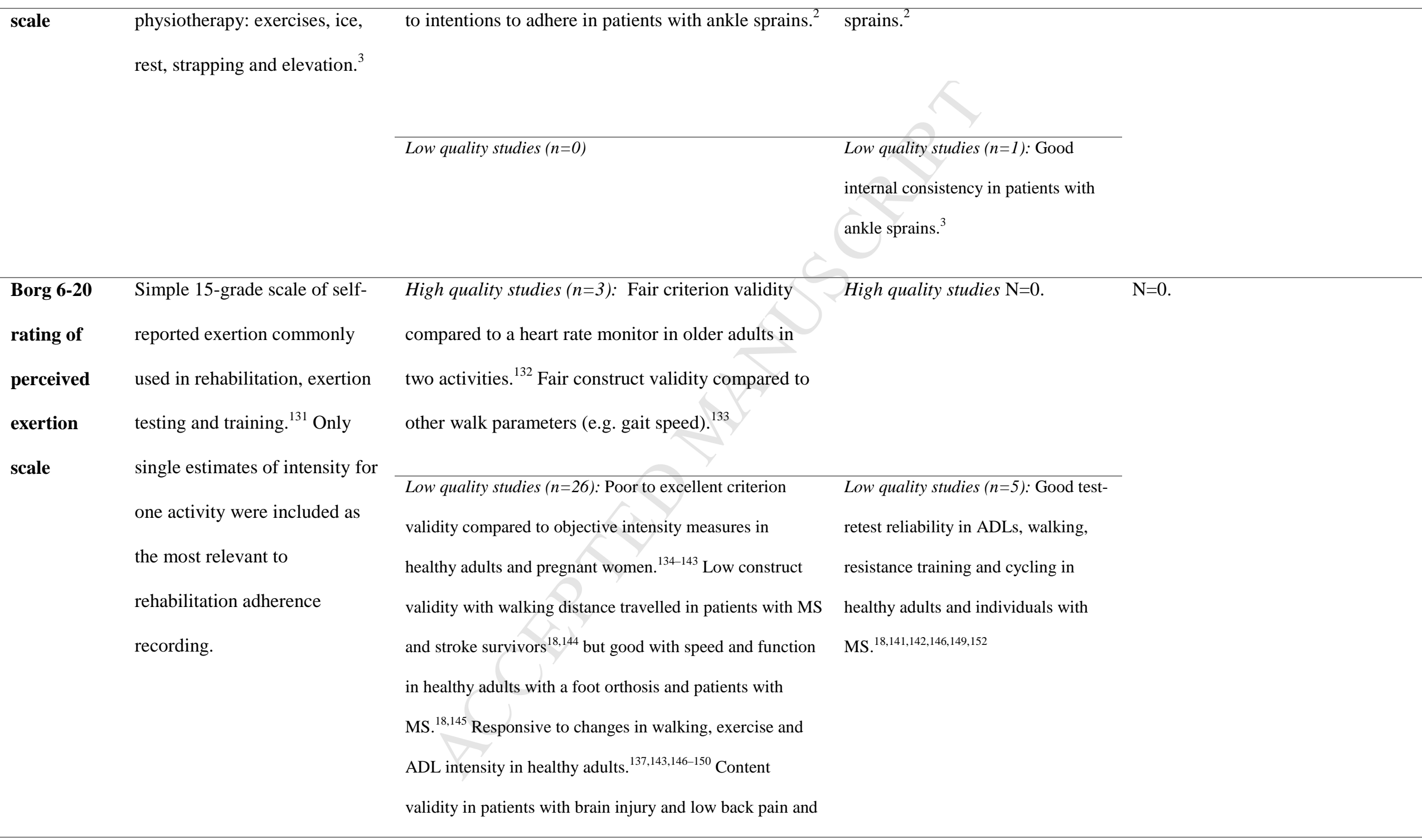


healthy students. ${ }^{151}$

\begin{tabular}{|c|c|c|c|c|}
\hline Yamax & Yamax pedometer which & High quality studies $(n=1)$ : Limited criterion validity & High quality studies $(n=0)$ & $\mathrm{N}=1 . \mathrm{CW}-701$ had data for $58 / 61$ \\
\hline Digiwalker & records and displays the & compared to a GT1M ActiGraph accelerometer in & & pregnant women for four days' \\
\hline CW series & number of steps taken. It has a & pregnant women (overcounted at high step rates and & & wear. $^{154}$ \\
\hline & two week memory and a three & undercounted at lo & & \\
\hline & year battery life. ${ }^{153}$ All CW & 'active' and 'inactive' classifications. ${ }^{154}$ & & \\
\hline & & Low quality studies $(n=1)$ : Poor criterion validity (high & Low quality studies $(n=1)$ Good inter- & \\
\hline & mechanisms and so all were & percentage error) ir & rater reliability in older adults. ${ }^{155}$ & \\
\hline Joint & 20-task observational scale & High quality studies $(n=0)$ & High quality studies $(n=0)$ & $\mathrm{N}=1.83 / 127$ individuals with \\
\hline Protection & assessing performance accuracy & & & rheumatoid arthritis agreed to be \\
\hline Behaviour & of arthritis joint protection & & & recorded performing the JPBA. ${ }^{12}$ \\
\hline Assessment & behaviours when making a hot & Low quality studies $(n=6)$ : Fair construct validity with & Low quality studies: Excellent test- & \\
\hline$($ JPBA) & drink and snack in a kitchen. ${ }^{156}$ & hand impairment, ${ }^{156,157}$ but higher with pain, perceived & retest, inter- and intra-rater reliability & \\
\hline & Behaviours are graded as & helplessness and reduced grip strength in persons with & and internal consistency in healthy & \\
\hline & correct, partially correct or & $\begin{array}{l}\text { rheumatoid arthritis. }{ }^{156,158} \text { Responsive to changes in joint } \\
\text { protection training in healthy adults. }{ }^{159} \text { Good face and }\end{array}$ & adults and individuals with & \\
\hline
\end{tabular}


incorrect and converted to a

percentage score. ${ }^{156}$

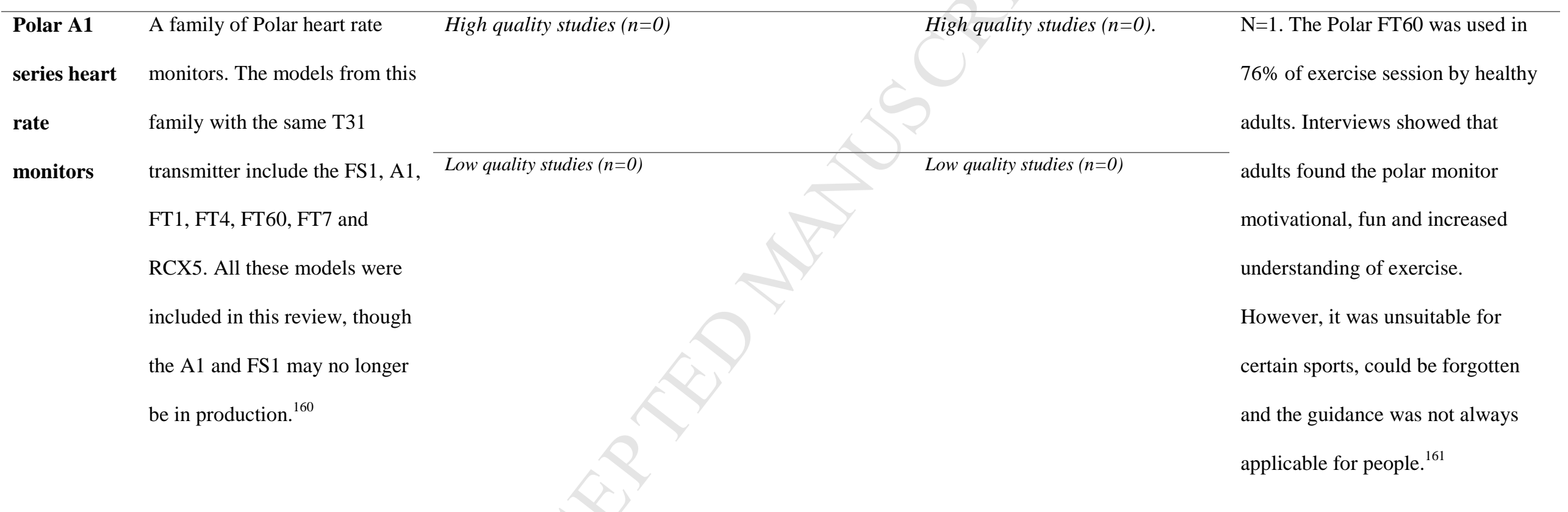

The reference list for included studies can be found in Supplementary File 3. 
Table 3. Implications for adherence measures identified in this review

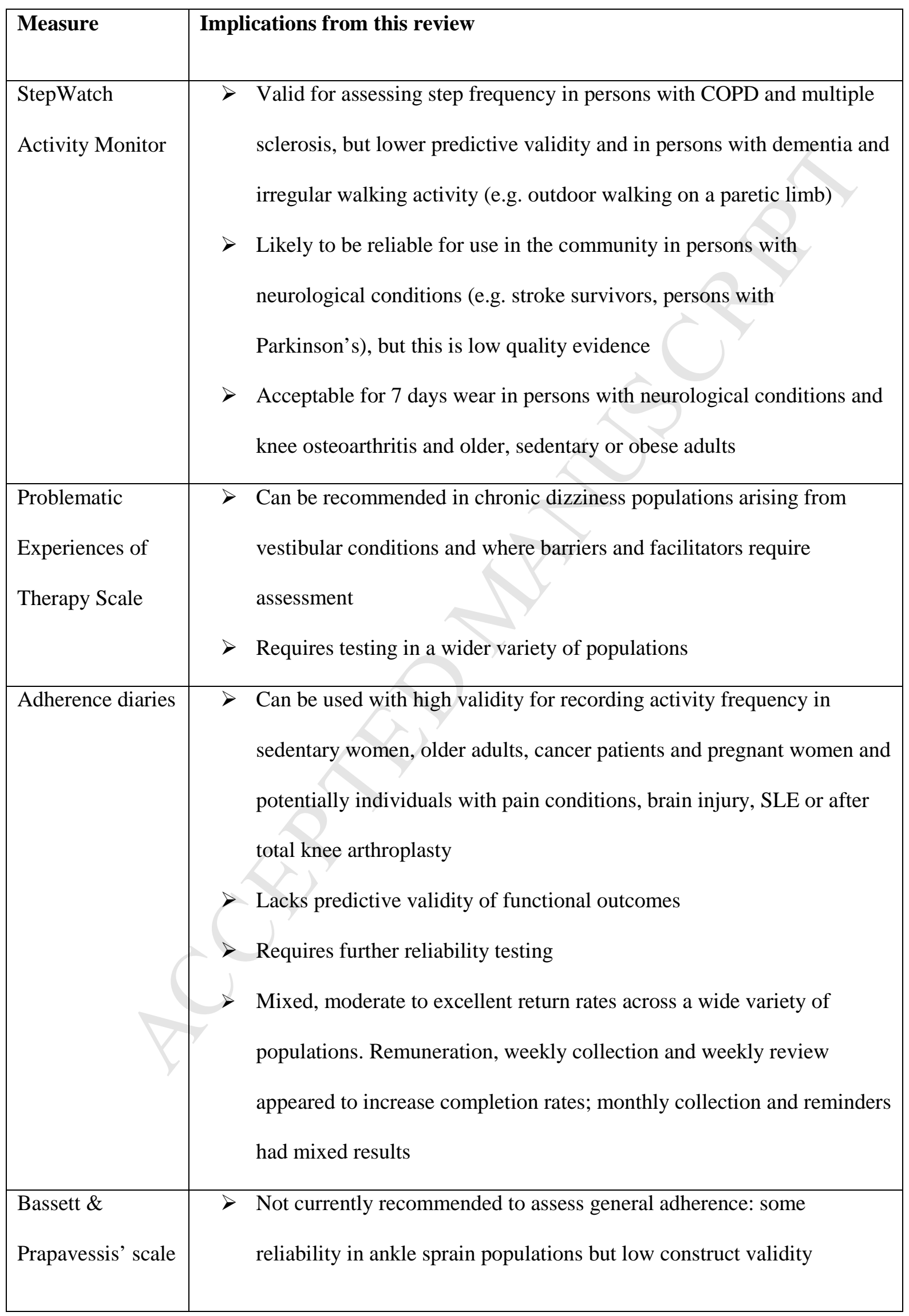




\begin{tabular}{|c|c|}
\hline $\begin{array}{l}\text { Borg 6-20 rating } \\
\text { of perceived } \\
\text { exertion scale }\end{array}$ & $\begin{array}{l}\text { Not currently recommended to assess intensity adherence: only fair } \\
\text { validity in older populations, though may be reliable and responsive to } \\
\text { change }\end{array}$ \\
\hline $\begin{array}{l}\text { Yamax } \\
\text { Digiwalker CW } \\
\text { series }\end{array}$ & $\begin{array}{l}\text { May be acceptable but cannot be recommended above other measures as } \\
\text { it lacks evidence of good validity } \\
>\text { Pedometer models with good supporting evidence should be selected }\end{array}$ \\
\hline $\begin{array}{l}\text { Joint Protection } \\
\text { Behaviour } \\
\text { Assessment }\end{array}$ & $\begin{array}{l}\text { Recommended for assessing accuracy adherence of joint protection } \\
\text { behaviour in patients with rheumatoid arthritis, though evidence is } \\
\text { limited }\end{array}$ \\
\hline $\begin{array}{l}\text { Polar A1 heart } \\
\text { rate monitor } \\
\text { series }\end{array}$ & $\begin{array}{l}\text { May be acceptable to healthy adults but not currently recommended due } \\
\text { to a lack of evidence } \\
>\text { Heart monitor models with good supporting evidence should be selected }\end{array}$ \\
\hline
\end{tabular}




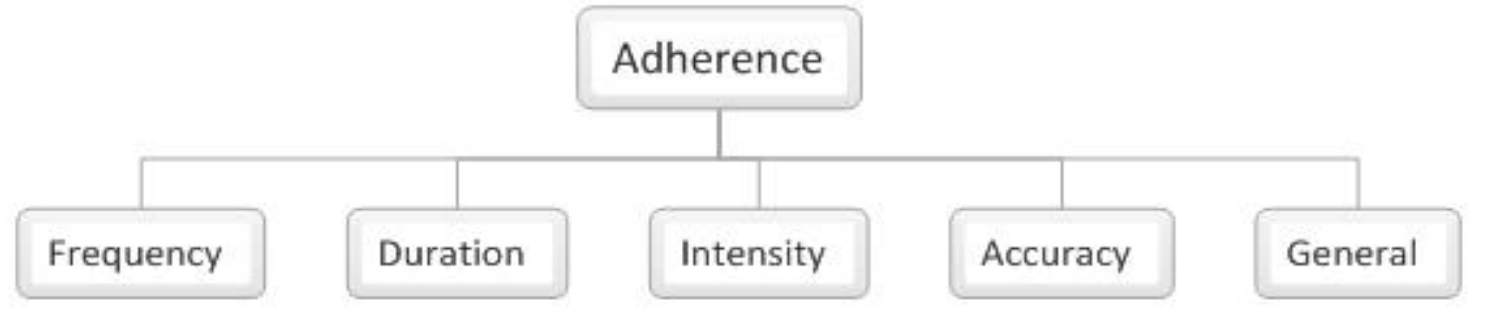




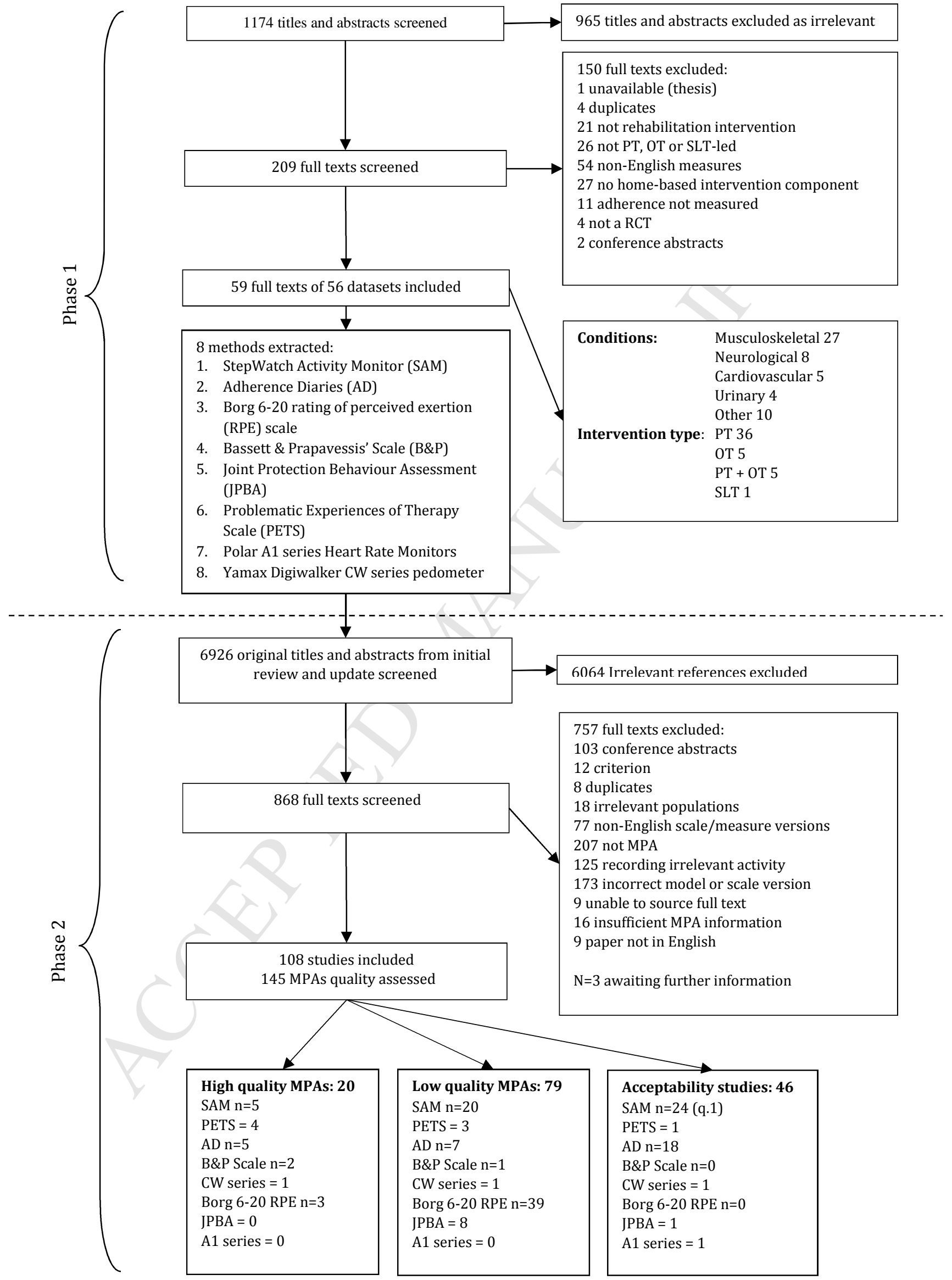


Supplementary File 2. Table of included validity, reliability and acceptability studies.

\begin{tabular}{|c|c|c|c|c|c|c|c|}
\hline Reference & MPA & Para & $\begin{array}{l}\text { Population (n, descriptor, mean (SD) } \\
\text { age, \%female) }\end{array}$ & Activity & Comparator & Statistic and outcome & Qual \\
\hline \multicolumn{8}{|c|}{ StepWatch Activity Monitor high quality studies $(n=5)$} \\
\hline $\begin{array}{l}\text { Feito } \\
2012 \mathbf{a}^{162}\end{array}$ & Crit val & $\mathrm{F}, \mathrm{I}$ & $\begin{array}{l}\mathrm{n}=65 \text {, healthy volunteers, full } \\
\text { sample }(\mathrm{n}=71) \text { : normal }=27.8 \mathrm{yrs} \\
(8.0) \text {, overweight }=34.6 \mathrm{yrs}(14.2) \text {, } \\
\text { obese }=31.5 \mathrm{yrs}(11.1), 55 \% \mathrm{f}\end{array}$ & $\begin{array}{l}\text { Lab; } 5 \text { minute walk on } \\
\text { motorized treadmill at } 3 \\
\text { different speeds }(40,67 \text { and } \\
94 \mathrm{~m} / \mathrm{min}) .\end{array}$ & $\begin{array}{l}\text { Trained observer with hand } \\
\text { tally counter }\end{array}$ & $\begin{array}{l}\text { Mean bias close to zero with } 95 \% \text { prediction } \\
\text { interval: } \pm 8 \text { steps } / \text { min } \\
95-102 \% \text { steps recorded across different } \\
\text { speeds. Pearson correlations: slow speed } \\
r=0.635 \text {, moderate speed } r=0.500 \text {, fast speed } \\
r=0.558 \text { (all } p<0.001 \text { ) }\end{array}$ & G \\
\hline $\begin{array}{l}\text { Feito } \\
2012 b^{163}\end{array}$ & Crit val & $\mathrm{F}, \mathrm{I}$ & $\begin{array}{l}\mathrm{n}=56 \text {, healthy individuals with a } \\
\text { range of BMI values, } \\
\text { normal=28.3yrs (10.5), } \\
\text { overweight }=31.2 \mathrm{yrs}(9.9) \\
\text { obese }=29.0 \mathrm{yrs}(7.9), 50 \% \mathrm{f}\end{array}$ & $\begin{array}{l}\text { Lab; } 5 \times 100 \text { step walks on a } \\
\text { treadmill at different speeds } \\
(40,54,67,80 \text { and } 94 \\
\mathrm{m} / \mathrm{min})\end{array}$ & $\begin{array}{l}\text { Trained observer with hand } \\
\text { tally counter }\end{array}$ & $\begin{array}{l}100 \pm 1 \% \text { accuracy at slowest speed, }>97 \% \\
\text { accuracy at faster speeds. No effect of BMI. }\end{array}$ & G \\
\hline Hiatt $2011^{48}$ & Crit val & $\mathrm{D}$ & $\begin{array}{l}\mathrm{n}=62 \text {, intermittent claudication } \\
\text { patients randomised to take } \\
\text { propionyl-L-carnitine or placebo, } \\
\text { G1 n=30, 66.6yrs (8.8), 17\%f, G2 n- } \\
3267.4 \mathrm{yrs}(8.7), 38 \% \mathrm{f}\end{array}$ & $\begin{array}{l}\text { Home/community; } 30-50 \\
\text { min walking 2-3 times per } \\
\text { week; daily activities for } 7 \\
\text { days at screening, } 3 \text { mo and } \\
6 \text { mo }\end{array}$ & $\begin{array}{l}\text { Change in Peak Walking } \\
\text { Time between baseline and } \\
6 \mathrm{mo}\end{array}$ & $\begin{array}{l}\text { Changes in SAM ambulatory activity } r=0.34 \\
(p=0.013) \\
\text { Changes in SAM dose (mins of exercise) } \\
r=0.259(p=0.048)\end{array}$ & G \\
\hline Moy 2012164 & Crit val & $\mathrm{F}$ & $\mathrm{n}=127$, stable COPD patients $>40$ & Lab; $244 \mathrm{~m}$ walking course & Observer & Mean bias ( $95 \%$ LOA): +3 steps $(-13.53$ to & G \\
\hline
\end{tabular}




\begin{tabular}{|c|c|c|c|c|c|c|c|}
\hline \multirow[t]{2}{*}{ Reference } & \multirow[t]{2}{*}{ MPA } & \multirow[t]{2}{*}{ Para } & \multirow{2}{*}{$\begin{array}{l}\text { Population (n, descriptor, mean (SD) } \\
\text { age, \%female) } \\
71.0 \mathrm{yrs}(8.0), 2 \% \mathrm{f}\end{array}$} & \multirow{2}{*}{$\begin{array}{l}\text { Activity } \\
\text { at usual speed }\end{array}$} & \multirow[t]{2}{*}{ Comparator } & \multirow{2}{*}{$\begin{array}{l}\text { Statistic and outcome } \\
20.11 \text { steps). }>90 \% \text { accuracy in } 133 / 134 \\
\text { participants. No effect of BMI. }\end{array}$} & \multirow[t]{2}{*}{ Qual } \\
\hline & & & & & & & \\
\hline $\begin{array}{l}\text { Sandroff } \\
201461\end{array}$ & Crit val & $\mathrm{F}$ & $\begin{array}{l}\mathrm{n}=63 \text {, ambulatory individuals with } \\
\text { multiple sclerosis, 50.7yrs (9.2), } \\
76 \% \text { f }\end{array}$ & $\begin{array}{l}\text { Lab; } 3 \times 6 \text { min walk test } \\
\text { around a rectangular } \\
\text { hallway at comfortable, fast } \\
\text { and slow walking speeds }\end{array}$ & $\begin{array}{l}\text { Direct observation by } \\
\text { research assistant using } \\
\text { hand tally counter }\end{array}$ & $\begin{array}{l}\text { Comfortable walking speed }=99.8 \% \\
\text { accuracy, fast } 99.9 \% \text {, slow } 99.0 \% \text {. High } \\
\text { disability and low speed were less accurate. }\end{array}$ & G \\
\hline StepWatch Acti & ity Monito & low qu & lity studies ( $n=35$ ) & & & & \\
\hline Algase $2003{ }^{77}$ & Crit val & $\mathrm{D}$ & $\begin{array}{l}\mathrm{N}=40 \text {, individuals with dementia, (all } \\
\text { subject } \mathrm{n}=178) 85.3(6.3), 75 \% \mathrm{f}\end{array}$ & $\begin{array}{l}\text { Nursing home; duration of } \\
\text { wandering in two } 4 \mathrm{hr} \text { periods }\end{array}$ & $\begin{array}{l}\text { Trained observers recording } \\
\text { using a bar code reader }\end{array}$ & $\begin{array}{l}\text { Multiple regression controlled for age, sex and } \\
\text { mini-mental state examination score: SAM } \\
\text { predicted } 63.6 \% \text { of the variance in time spent } \\
\text { wandering ( } \mathrm{p}<0.001) \text {. Time in motion } 16.8 \% \text { SAM } \\
\text { vs } 15.4 \% \text { observation. }\end{array}$ & $\mathrm{P}$ \\
\hline $\begin{array}{l}\text { Bergman } \\
2008^{165}\end{array}$ & Crit val & $\mathrm{F}$ & $\begin{array}{l}\mathrm{N}=21 \text {, older adults living in assisted } \\
\text { living facilities, } 78.6(13.1), 76 \% \mathrm{f}\end{array}$ & $\begin{array}{l}\text { Assisted living facility; walking } \\
\text { course, } 161 \mathrm{~m} \text { walk at a self- } \\
\text { selected pace }\end{array}$ & $\begin{array}{l}\text { Observer with hand tally } \\
\text { counter }\end{array}$ & $\begin{array}{l}\text { Mean bias }=-11.3 \text { (SE } 2.56) \text { (overestimation) } \\
(p<0.001), 95 \% \text { prediction interval }=-18.01 \text { to }- \\
4.65 . \\
\text { Correlations } r^{2}=0.99(p<0.001)\end{array}$ & $\mathrm{P}$ \\
\hline $\begin{array}{l}\text { Bowden } \\
2007^{101}\end{array}$ & Crit val & $\mathrm{F}$ & $\begin{array}{l}\mathrm{n}=11 \text {, individuals with incomplete } \\
\text { spinal cord injury with no more than } \\
\text { minimal assistance required for } \\
\text { walking, } 45.5 \text { (range } 21-63 \text { ), } 18 \% \mathrm{f}\end{array}$ & $\begin{array}{l}\text { Lab; } 1 \times 6 \text { minute walk test at } \\
\text { usual pace over series of } \\
\text { hallways, } 2 \times 10 \text { minute walk } \\
\text { tests at self-selected pace, } \\
\text { completed at } 2 \text { different times }\end{array}$ & $\begin{array}{l}\text { Observer with manual } \\
\text { handheld counter }\end{array}$ & $\begin{array}{l}\text { Percentage accuracy (smaller quantity as } \\
\text { percentage of larger quantity) } 6 \text { minute walk test } \\
=97 \%, 10 \text { minute walk test }=97 \%\end{array}$ & $\mathrm{P}$ \\
\hline
\end{tabular}




\begin{tabular}{|c|c|c|c|c|c|c|c|}
\hline Reference & MPA & Para & $\begin{array}{l}\text { Population (n, descriptor, mean (SD) } \\
\text { age, \%female) }\end{array}$ & Activity & Comparator & Statistic and outcome & Qual \\
\hline & & & & $\begin{array}{l}\text { in randomised order ( } 4 \mathrm{hrs} \mathrm{-} 1 \\
\text { week later) }\end{array}$ & & & \\
\hline Busse $2009^{85}$ & Crit val & $\mathrm{F}$ & $\begin{array}{l}n=18 \text {, healthy volunteers, } 26.1 \text { (range } \\
22-39 \text { ), gender NR }\end{array}$ & $\begin{array}{l}\text { Lab; Walking an indoor circuit } \\
\text { for } \sim 10 \text { mins }(200 \mathrm{~m}) \text {, including } \\
\text { sit-to-stand transitions, } \\
\text { completion of kitchen tasks and } \\
\text { shoe removal; and an outdoor } \\
\text { circuit for } \sim 20 \mathrm{mins}(1100 \mathrm{~m}) \text {, } \\
\text { including uneven ground, lifts, } \\
\text { ramps }\end{array}$ & $\begin{array}{l}\text { Observer of videotaped walk } \\
\text { using handheld step counter by } \\
\text { one researcher with excellent } \\
\text { intra-rater reliability. Overall } \\
\text { ICC }=0.99 \text { for intra-rater } \\
\text { reliability, but poor inter-rater } \\
\text { reliability (ICC } 0.26 \text { ) }\end{array}$ & $\begin{array}{l}\text { Indoor: } \text { mean (SD) dif }=5.76 \%(5.18) . \mathrm{LOA}=-4.6 \\
\text { to } 16.2 \text { steps. } \\
\text { Outdoor: mean (SD) dif = } 2.82 \%(7.47) . \mathrm{LOA}-12.2 \\
\text { to } 17.8 \text { steps. } \\
\text { Percentage accuracy: Indoor = 96.1\% (3.5), } \\
\text { outdoor = 99.6\% (1.1). } \\
\text { Percentage error: indoor }=3.9 \%(3.5) \text {, outdoor }= \\
0.4 \% \text { (1.1) }\end{array}$ & $\mathrm{P}$ \\
\hline Carr 2012 ${ }^{86}$ & Crit val & D, I & $\mathrm{N}=36$, healthy adults, 23 (3.7), 55\%f & $\begin{array}{l}\text { Lab; } 60 \mathrm{~min} \text { testing session } \\
\text { including } 6 \text { sedentary and light } \\
\text { activity activities for } 8 \text { min each } \\
\text { (middle } 6 \text { min compared) }\end{array}$ & $\begin{array}{l}\text { Observer watching activities for } \\
\text { fidelity }\end{array}$ & $\begin{array}{l}\text { Percentage accuracy for light intensity: } \\
\text { Walking } 1.0 \mathrm{mph}: 86.1 \% \text {. Pedalling } 7.0 \mathrm{mph} 54.4 \% \text {. } \\
\text { Pedalling } 15.0 \mathrm{mph} 23.5 \% \text {. Root mean square error } \\
\text { for minutes correctly coded }=3.33 \mathrm{~min}\end{array}$ & $\mathrm{~F}$ \\
\hline Ford $2010^{92}$ & Crit val & $\mathrm{F}$ & $\begin{array}{l}\mathrm{n}=12 \text {, individuals with Parkinson's } \\
\text { disease, } 67.2 \text { (SD NR), 8.3\%f }\end{array}$ & Lab; 1 min walk around the lab & Single observer & Percentage accuracy $98 \%$ & $\mathrm{P}$ \\
\hline Foster $2005^{87}$ & Crit val & $\mathrm{F}$ & $\begin{array}{l}\mathrm{n}=20 \text {, healthy adults } 50 \% \text { lean } 30(13) \text {, } \\
50 \% \text { obese } 32(7) \text {, age range }=21-51 \mathrm{yrs} \text {, } \\
50 \% \mathrm{f}\end{array}$ & $\begin{array}{l}\text { Lab; } 15 \mathrm{~min} \text { walks at } 1,2 \text { and } 3 \\
\text { mph each. Level ground } \\
\text { walking at } 1 \text { and } 1.85 \mathrm{mph} \text { each } \\
\text { for } 25 \mathrm{~min} .\end{array}$ & $\begin{array}{l}\text { Single observer using electronic } \\
\text { counter }\end{array}$ & $\begin{array}{l}\text { Percentage accuracy } 99.7 \% \pm 0.67 \\
\text { ICC }=0.9995\end{array}$ & $\mathrm{P}$ \\
\hline
\end{tabular}




\begin{tabular}{|c|c|c|c|c|c|c|c|}
\hline Reference & MPA & Para & $\begin{array}{l}\text { Population (n, descriptor, mean (SD) } \\
\text { age, \%female) }\end{array}$ & Activity & Comparator & Statistic and outcome & Qual \\
\hline & & & $\begin{array}{l}\text { brain injury, able to walk with minimal } \\
\text { assistance, able to follow study } \\
\text { commands and give informed consent. } \\
\text { (full sample n=50) } 52.9 \text { (15.1), 32\%f }\end{array}$ & $\begin{array}{l}\text { normal, comfortable pace with } \\
\text { SAM on less affected side }\end{array}$ & $\begin{array}{l}\text { walk on two separate occasions } \\
\text { (ICC }=0.99 \text { ), with first count used } \\
\text { in analysis }\end{array}$ & $\begin{array}{l}\text { relationship between SAM error and gait speed, } \\
\text { Berg balance scale or Fugl-Meyer score } \\
\text { ICC }=0.97(0.92-0.99)\end{array}$ & \\
\hline $\begin{array}{l}\text { Hartsell } \\
2002^{88}\end{array}$ & Crit val & $\mathrm{F}$ & $\begin{array}{l}\mathrm{N}=10 \text {, healthy adults, } 43.2 \text { (14.1), mixed } \\
\text { (NR). }\end{array}$ & $\begin{array}{l}\text { Lab; walking course; } 4 \times 530 \mathrm{~m} \\
\text { walk in athletic shoes or } \\
\text { fibreglass cast (TCC) on one leg, } \\
\text { over flat ground and stairs each } \\
\text { at self-selected pace }\end{array}$ & $\begin{array}{l}\text { Mean of } 2 \text { observers }(r=0.9923 \text { - } \\
0.9999)\end{array}$ & $\begin{array}{l}\text { Percentage error: flat surface: athletic shoe } \\
0.136 \% \text {, TCC } 0.206 \% \text {. Stairs: }-3.648 \% \text { athletic shoe } \\
\text { and }-5.697 \% \text { TCC (undercounting). } \\
\text { ANOVA: significant effects for walking surfaces. }\end{array}$ & $\mathrm{P}$ \\
\hline $\begin{array}{l}\text { Karabulut } \\
2005^{89}\end{array}$ & Crit val & F, I, & $\mathrm{N}=20$, healthy adults, 28 (3.7), $50 \% \mathrm{f}$ & $\begin{array}{l}\text { Lab; treadmill; 3min walks at a } \\
\text { variety of speeds, 3min each of } \\
\text { heel tapping, leg swinging, cycle } \\
\text { ergometer and }(n=10) \text { driving. }\end{array}$ & $\begin{array}{l}\text { Observer with hand tally } \\
\left.\text { counter ( } 2^{\text {nd }} \text { min only }\right)\end{array}$ & $\begin{array}{l}\text { Mean bias }=0.9{\text { steps } \mathrm{min}^{-1} \text {, prediction interval }=-} \\
2.3 \text { to }+4.1 \text { steps } \mathrm{min}^{-1} . \text { Mean step counts within } \\
1 \% \text { at all speeds. SAM responsive to heel tapping, } \\
\text { leg swinging and cycling but not driving. }\end{array}$ & $\mathrm{P}$ \\
\hline Macko 2002 ${ }^{94}$ & Crit val & $\mathrm{F}$ & $\begin{array}{l}\mathrm{n}=16,>55 \text { yrs of age with remote } \\
\text { ischemic stroke ( }>6 \text { months), with } \\
\text { residual hemiparetic gait deficits and } \\
\text { some preserved capacity for } \\
\text { ambulation, } 67 \text { (7), mixed (NR) }\end{array}$ & $\begin{array}{l}\text { Rehabilitation centre; walking } \\
\text { course; } 2 \times 6 \mathrm{~min} \text { floor walk at } \\
\text { self-selected pace, } 2 \times 1 \mathrm{~min} \text { floor } \\
\text { at self-selected comfortable and } \\
\text { fastest pace using normal } \\
\text { adaptive device/orthosis }\end{array}$ & Observer with hand tally & $\begin{array}{l}\text { Percentage accuracy: } \\
\text { self-selected pace } 98.5 \% \pm-1.0(\mathrm{P}<0.01) \text {, } \\
\text { fast walking pace } 97.7 \pm-2.0^{*}(\mathrm{p}<0.01) \\
\text { First } 6 \text { min walk } 98.8 \pm 1.1 \\
2^{\text {nd }} 6 \text { min walk } 98.7 \pm 1.2\end{array}$ & $\mathrm{P}$ \\
\hline $\begin{array}{l}\text { Mudge } \\
2007^{166}\end{array}$ & Crit val & $\mathrm{F}$ & $\begin{array}{l}\mathrm{n}=25 \text { chronic stroke patients, median } \\
\text { age }=69 \text { (range } 42-79), 32 \% \mathrm{f}\end{array}$ & $\begin{array}{l}\text { Lab; } 6 \text { trials on a } 6 \mathrm{~m} \text { walkway } \\
\text { without shoes at a self-selected }\end{array}$ & 3-dimensional gait analysis & $\begin{array}{l}\text { Pearson's r }=0.959 \text { (non-paretic limb) and r=0.896 } \\
\text { (paretic limb) }\end{array}$ & $\mathrm{P}$ \\
\hline
\end{tabular}


age, \%female)

pace

\begin{tabular}{|c|c|c|c|c|c|c|c|}
\hline & Crit val & $\mathrm{F}$ & $\begin{array}{l}\mathrm{n}=21 \text {, chronic stroke patients, full } \\
\text { sample median age = } 69 \text { (range } 42-79 \text { ), } \\
32 \% \mathrm{f}\end{array}$ & $\begin{array}{l}\text { Lab; indoor: } 8 \mathrm{~m} \text { at self-selected } \\
\text { pace and } 8 \mathrm{~m} \text { at fast pace, } \\
\text { outdoor: } 200 \mathrm{~m} \text { course including } \\
\text { steps, inclines and declines } \\
\text { wearing usual footwear at a } \\
\text { self-selected pace (with rest if } \\
\text { required). }\end{array}$ & $\begin{array}{l}\text { On/off event footswitches } \\
\text { taped to the foot }\end{array}$ & $\begin{array}{l}95 \% \text { LOA: non-paretic limb } \pm 9 \text { steps, paretic limb } \\
\pm 57 \text { steps } \\
\text { Percentage error: non-paretic limb }-1.3 \% \text { (range, }- \\
4.5 \% \text { to } 2.5 \% \text { ), paretic limb }-4.2 \% \text { (range, }-42 \% \text { to } \\
16 \% \text { ). } \\
\text { Pearson correlations: non-paretic limb r=0.999, } \\
\text { paretic limb r }=0.963\end{array}$ & $P$ \\
\hline Ng 2012 167 & Crit val & F, I & $\begin{array}{l}\mathrm{N}=20 \text {, chronic obstructive pulmonary } \\
\text { disease patients with functional } \\
\text { limitation, } 73(8.5), 60 \% \mathrm{f}\end{array}$ & $\begin{array}{l}\text { Lab; walking course; self- } \\
\text { selected slow and normal paces } \\
\text { with and without a rollator for } \\
\text { 5min each. }\end{array}$ & $\begin{array}{l}\text { Observer (average of } 30 \mathrm{~s} \\
\text { interval at start of } 2^{\text {nd }}, 3^{\text {rd }} \text { and } \\
4^{\text {th }} \text { minute) }\end{array}$ & $\begin{array}{l}\text { Mean bias }=+2 \text { steps } / \mathrm{min}, 95 \% \text { LOA } 6 \text { steps } / \mathrm{min} \\
\text { ( }-4 \text { to } 8 \text { steps } / \mathrm{min} \text { ). } \\
\text { No effect of rollator or walking speed on validity of } \\
\text { step rate. }\end{array}$ & $\mathrm{P}$ \\
\hline $\begin{array}{l}\text { Resnick } \\
2001^{97}\end{array}$ & Crit val & $\mathrm{F}$ & $\begin{array}{l}N=30 \text {, older adults }(65+) \text { with } \\
\text { Parkinson's ( } n=3) \text {, previous hip fracture } \\
\text { ( } n=10 \text { ) or evidence of degenerative joint } \\
\text { disease and/or osteoporosis ( } n=17), 86 \\
\text { (6.1), 73\%f }\end{array}$ & $\begin{array}{l}\text { Lab; } 1 \text { min walk at self-selected } \\
\text { speed over carpet, repeated } \\
\text { after a } 2 \mathrm{~min} \text { rest }\end{array}$ & $\begin{array}{l}\text { Mean of two observers } \\
\text { (experienced nurses). Inter- } \\
\text { rater reliability }=0.98\end{array}$ & $\begin{array}{l}\text { Correlations (type not stated) } \mathrm{r}=0.95(\mathrm{p}<0.05) \\
\% \text { accuracy }=96 \% \\
\% \text { error }=4.0 \pm 3.1 \% \text { (range } 0-12 \%)\end{array}$ & F \\
\hline $\begin{array}{l}\text { Schmidt } \\
2011^{168}\end{array}$ & Crit val & $\mathrm{F}$ & $\begin{array}{l}\mathrm{N}=20 \text {, individuals with Parkinson's } \\
\text { disease ( } \mathrm{n}=11,66.8 \text { (SD NR)) or } \\
\text { multiple sclerosis ( } \mathrm{n}=9,55.8 \text { (SD NR)), }\end{array}$ & $\begin{array}{l}\text { Lab; walking course; } 3 \text { walks at } \\
\text { usual speed over the GaitMat II }\end{array}$ & GaitMat II & $\begin{array}{l}\text { Pearson correlations: Multiple sclerosis r=0.99, } \\
\text { Parkinson's disease } r=1.0 \text {. } \\
\text { Mean strides: } 15.55 \text { (SAM) and } 15.85 \text { (GM). }\end{array}$ & $\mathrm{P}$ \\
\hline
\end{tabular}




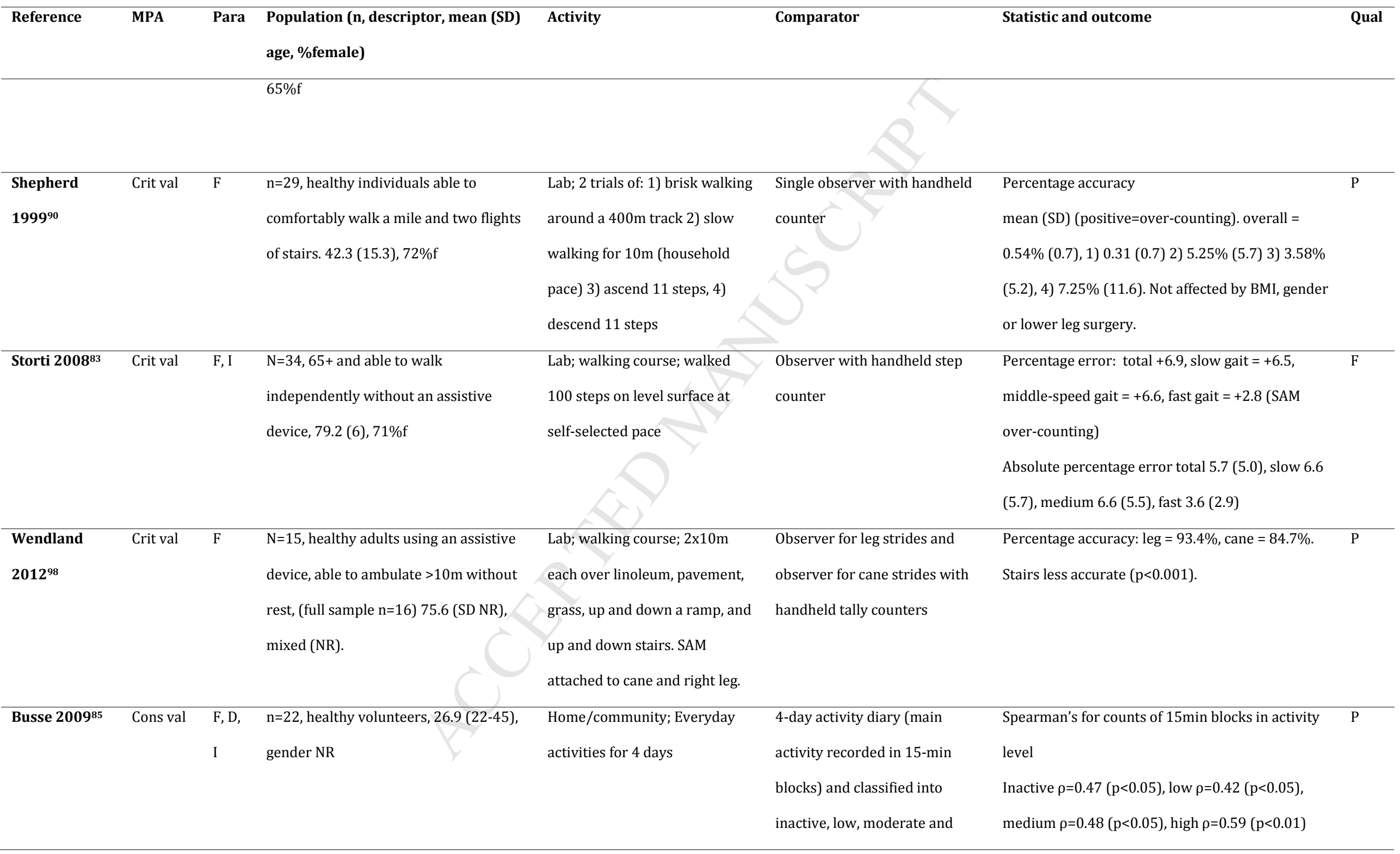




\begin{tabular}{|c|c|c|c|c|c|c|c|}
\hline Reference & MPA & Para & $\begin{array}{l}\text { Population (n, descriptor, mean (SD) } \\
\text { age, \%female) }\end{array}$ & Activity & Comparator & Statistic and outcome & Qual \\
\hline & & & & & vigorous based on METs & & \\
\hline $\begin{array}{l}\text { Bowden } \\
2007^{91101}\end{array}$ & TRR & $\mathrm{F}$ & $\begin{array}{l}\mathrm{n}=11 \text {, diagnosis of incomplete spinal } \\
\text { cord injury with no more than minimal } \\
\text { assistance required for walking, } 45.5 \mathrm{yrs} \\
\text { (range } 21-63 \text { ), } 18.2 \% \mathrm{f}\end{array}$ & $\begin{array}{l}\text { Lab; } 1 \times 6 \mathrm{MWT} \text { at usual pace } \\
\text { over series of hallways, } 2 \times 10 \mathrm{~m} \\
\text { WTs at self-selected pace, } \\
\text { completed at } 2 \text { different times } \\
\text { in randomised order }\end{array}$ & $\begin{array}{l}4 \text { hrs to } 1 \text { week later both tests } \\
\text { repeated }\end{array}$ & ICC $(2,1) 6 \mathrm{MWT}=0.99,10 \mathrm{mWT}=0.97$ & $\mathrm{P}$ \\
\hline Busse 200985 & TRR & $\mathrm{F}$ & $\begin{array}{l}\mathrm{n}=20 \text {, healthy volunteers, } 26.15 \text { (range } \\
\text { 17-38), gender NR }\end{array}$ & $\begin{array}{l}\text { Lab; } 3 \text { outdoor } 20 \text { min circuit } \\
\text { walks with ramps, lifts etc using } \\
\text { metronome to standardise } \\
\text { cadence }\end{array}$ & Three walks of same circuit & ICC $=0.96$ & $\mathrm{P}$ \\
\hline Busse 2004 ${ }^{62}$ & TRR & $\begin{array}{l}\mathrm{F}, \mathrm{D}, \\
\mathrm{I}\end{array}$ & $\begin{array}{l}\mathrm{n}=10 \text { healthy adults, } 43.3(18.9), 40 \% \mathrm{f} \\
\mathrm{n}=10 \text { ambulant neurological patients } \\
\text { with impairments from different } \\
\text { pathologies with restricted walking } \\
\text { mobility but able to walk }>10 \mathrm{~m} \text { without } \\
\text { assistance, } 59.4(13.4), 50 \% \mathrm{f}\end{array}$ & $\begin{array}{l}\text { Home/community; Everyday } \\
\text { activities for two 7-day } \\
\text { monitoring periods (SAM worn } \\
\text { for } 24 \mathrm{hr} / \text { day ad removed for } \\
\text { bathing) }\end{array}$ & 1-3 weeks apart & $\begin{array}{l}\text { Healthy step count: } \mathrm{ICC}=0.89 \text { day to day } \mathrm{CV}=28 \% \text {, } \\
\text { week to week CV } 8.8 \% \text {. Peak activity index } \\
\text { ICC }=0.98 .20 \text { min sustained activity ICC }=0.75 \text {. } \\
30 \mathrm{~min} \text { sustained activity ICC }=0.71,60 \mathrm{~min} \\
\text { sustained activity }=0.57 \text {. } \\
\text { Neurological patients step count ICC=0.86 day to } \\
\text { day CV=30\%, week to week } 12 \% \text {. Peak activity } \\
\text { index ICC=0.82, } 20 \text { min sustained activity ICC=0.94. } \\
\text { 30min sustained activity ICC=0.90, } 60 \mathrm{~min} \\
\text { sustained activity }=0.95 \text {. }\end{array}$ & $\mathrm{P}$ \\
\hline Haeuber & TRR & $\mathrm{F}$ & $\mathrm{n}=17,>50$, remote ischaemic stroke & Home/community; total strides & Average per day of two $48 \mathrm{hr}$ & ICC $=0.96(\mathrm{p}<0.001)$ & $\mathrm{P}$ \\
\hline
\end{tabular}




\begin{tabular}{|c|c|c|c|c|c|c|c|}
\hline Reference & MPA & Para & $\begin{array}{l}\text { Population (n, descriptor, mean (SD) } \\
\text { age, \%female) }\end{array}$ & Activity & Comparator & Statistic and outcome & Qual \\
\hline $2004^{169}$ & & & $\begin{array}{l}\text { over } 6 \mathrm{mth} \text { ago, residual hemiparetic } \\
\text { gait defects but capacity for ambulation } \\
\text { with assistive device. } 65 \text { (6), gender NR }\end{array}$ & over 48 hours & periods, up to 3 weeks' apart & & \\
\hline Algase $2003^{77}$ & TRR & $\mathrm{D}$ & $\begin{array}{l}\text { Sample size not clear, individuals with } \\
\text { dementia, (full sample } n=178 \text { ) } 85.3 \\
(6.3), 75 \% \mathrm{f}\end{array}$ & $\begin{array}{l}\text { Nursing home; free-living } \\
\text { wandering in 1-4 four-hour } \\
\text { periods }\end{array}$ & Time interval: 3 days & Pearson's correlations $r=0.71(p<0.001)$ & $\mathrm{F}$ \\
\hline Mudge 200875 & TRR & F, I & $\begin{array}{l}\mathrm{N}=40,>6 \text { months post-stroke, able to } \\
\text { walk independently but with some } \\
\text { residual difficulty, } 69.2 \text { (12.6), } 43 \% \mathrm{f}\end{array}$ & $\begin{array}{l}\text { Home/community; mean steps } \\
\text { in free-living three day period }\end{array}$ & $\begin{array}{l}\text { Time interval: } 1 \text { week (same 3- } \\
\text { day period) }\end{array}$ & $\begin{array}{l}\text { Total step count } \mathrm{ICC}=0.989 ; \mathrm{CV}=10.7 \% \text {; } \\
\text { Medium rate steps: } \mathrm{ICC}=0.964 ; \mathrm{CV}=17.8 \% \text {; } \\
\text { High rate steps: } \mathrm{ICC}=0.926 ; \mathrm{CV}=37.6 \% \text {; } \\
\text { Low rate steps: } \mathrm{ICC}=0.953 ; \mathrm{CV}=11.1 \%\end{array}$ & $\mathrm{~F}$ \\
\hline Macko $2002^{94}$ & TRR & $\mathrm{F}$ & $\begin{array}{l}\mathrm{N}=16 \text {, patients }>55 \text { yrs with remote } \\
\text { ischemic stroke ( }>6 \text { months), with } \\
\text { residual hemiparetic gait deficits and } \\
\text { some preserved capacity for } \\
\text { ambulation, } 67 \text { (7), mixed (NR). }\end{array}$ & $\begin{array}{l}\text { Rehabilitation centre; walking } \\
\text { course; } 2 \times 6 \mathrm{~min} \text { walks at self- } \\
\text { selected pace using their } \\
\text { normal adaptive } \\
\text { device/orthosis }\end{array}$ & Time interval: >=1 day (NR) & ICC $r=0.975, P<0.0001$ & $\mathrm{P}$ \\
\hline $\begin{array}{l}\text { Mudge } \\
2010^{170}\end{array}$ & TRR & F, I & $\begin{array}{l}\mathrm{N}=15 \text {, healthy adults, (full sample } \mathrm{n}=30 \text { ) } \\
27.7(8.9), 50 \% \mathrm{f}\end{array}$ & $\begin{array}{l}\text { Home/community; } 3 \text { days free- } \\
\text { living activity }\end{array}$ & $\begin{array}{l}\text { Time interval: } 1 \text { week (same 3- } \\
\text { day period) }\end{array}$ & $\begin{array}{l}\text { Mean steps/day: ICC=0.895; CV=11.8\%; } \\
\text { Medium rate steps: ICC=0.854; CV=13.0\%; } \\
\text { High rate steps: ICC=0.744; CV=36.9\% }\end{array}$ & $P$ \\
\hline $\begin{array}{l}\text { Resnick } \\
2001^{97}\end{array}$ & TRR & $\mathrm{F}$ & $\begin{array}{l}\mathrm{N}=30 \text {, older adults }(65+) \text { with } \\
\text { Parkinson's ( } \mathrm{n}=3 \text { ), previous hip fracture }\end{array}$ & $\begin{array}{l}\text { Lab; } 1 \text { min walk at self-selected } \\
\text { speed over carpet, repeated }\end{array}$ & Time interval: $2 \mathrm{~min}$ & ICC $r=0.84$ & $\mathrm{~F}$ \\
\hline
\end{tabular}




\begin{tabular}{|c|c|c|c|c|c|c|c|}
\hline Reference & MPA & Para & $\begin{array}{l}\text { Population (n, descriptor, mean (SD) } \\
\text { age, \%female) }\end{array}$ & Activity & Comparator & Statistic and outcome & Qual \\
\hline & & & $\begin{array}{l}\text { ( } n=10) \text { or evidence of degenerative joint } \\
\text { disease and/or osteoporosis ( } n=17), 86 \\
(6.1), 73 \% \mathrm{f}\end{array}$ & after a 2 min rest & & & \\
\hline $\begin{array}{l}\text { Subramony } \\
2012^{72}\end{array}$ & TRR & $\begin{array}{l}\text { F, D, } \\
\text { I }\end{array}$ & $\begin{array}{l}\mathrm{N}=19 \text {, ambulatory (with/without an } \\
\text { assistive device) individuals with } \\
\text { different spinocerebellar ataxias, } 56 \\
\text { (10.7), 79\%f }\end{array}$ & $\begin{array}{l}\text { Home/ community; free-living } \\
\text { wear for } 8 \text { days }\end{array}$ & $\begin{array}{l}\text { Time interval: days } 1-3 \\
\text { compared to days 5-7 }\end{array}$ & $\begin{array}{l}\text { Percentage time in activity: low speed ICC }=0.872 \text {, } \\
\text { moderate speed ICC }=0.886 \text {, high speed ICC }=0.606 \text {. } \\
\text { Percentage steps: low speed ICC }=0.912 \text {, moderate } \\
\text { speed ICC }=0.893 \text {, high speed ICC=0.793. } \\
\text { Average daily step count ICC=0.900. } \\
\text { Steps } / \text { min ICC=0.864. }\end{array}$ & $P$ \\
\hline Foster $2005^{87}$ & $\begin{array}{l}\text { Inter- } \\
\text { rater rel }\end{array}$ & $\mathrm{F}$ & $\begin{array}{l}\mathrm{n}=20 \text {, healthy adults } 50 \% \text { lean } 30(13) \text {, } \\
50 \% \text { obese } 32(7) \text {, age range }=21-51 \mathrm{yrs} \text {, } \\
50 \% \mathrm{f}\end{array}$ & $\begin{array}{l}\text { Lab; } 15 \mathrm{~min} \text { walks at } 1,2 \text { and } 3 \\
\text { mph each. Level ground } \\
\text { walking at } 1 \text { and } 1.85 \mathrm{mph} \text { each } \\
\text { for } 25 \mathrm{~min} \text {. }\end{array}$ & $\begin{array}{l}\text { SAM worn on inside of left } \\
\text { ankle and outside of right ankle } \\
\text { during same trials. }\end{array}$ & $\begin{array}{l}\text { Mean bias } 0.18 \pm 0.28 \text { steps } / \mathrm{min} \text { at } 1 \mathrm{ph}, 0.18 \pm 0.31 \\
\text { steps } / \mathrm{min} \text { at } 2 \mathrm{mph} \text {, and } 0.04 \pm 0.06 \mathrm{steps} / \mathrm{min} \text { at } 3 \\
\text { mph. Hall walking }=0.02 \mathrm{steps} / \mathrm{min} \text { compared to } \\
\text { treadmill measures }\end{array}$ & $P$ \\
\hline $\begin{array}{l}\text { Bowden } \\
2007^{101}\end{array}$ & ME & $\mathrm{F}$ & $\begin{array}{l}\mathrm{n}=11 \text {, diagnosis of incomplete spinal } \\
\text { cord injury with no more than minimal } \\
\text { assistance required for walking, range } \\
21-63,18.2 \% \mathrm{f}\end{array}$ & $\begin{array}{l}\text { Lab; } 1 \times 6 \text { minute walk test at } \\
\text { usual pace over series of } \\
\text { hallways, } 2 \times 10 \text { minute walk } \\
\text { test at self-selected pace, } \\
\text { completed at } 2 \text { different times } \\
\text { in randomised order ( } 4 \mathrm{hrs} \mathrm{-} 1 \\
\text { week later) }\end{array}$ & $\begin{array}{l}4 \mathrm{hrs} \text { to } 1 \text { week later both tests } \\
\text { repeated }\end{array}$ & $\begin{array}{l}\text { Standard error of measurement } \\
6 \text { minute walk test }=6.0 \text { steps } \\
10 \text { minute walk test }=0.76 \text { steps }\end{array}$ & $\mathrm{P}$ \\
\hline Mudge $2008^{75}$ & ME & $\mathrm{F}, \mathrm{I}$ & $\mathrm{N}=40,>6$ months post-stroke, able to & Home/community; free-living & Time interval: 1 week (same 3- & 95\% LOA (absolute,\%): & $\mathrm{F}$ \\
\hline
\end{tabular}




\begin{tabular}{|c|c|c|c|c|c|c|c|}
\hline Reference & MPA & Para & $\begin{array}{l}\text { Population (n, descriptor, mean (SD) } \\
\text { age, \%female) }\end{array}$ & Activity & Comparator & Statistic and outcome & Qual \\
\hline & & & $\begin{array}{l}\text { walk independently but with some } \\
\text { residual difficulty, } 69.2(12.6), 43 \% \mathrm{f}\end{array}$ & mean steps over 3 days; & day period) & $\begin{array}{l}\text { Total } 3 \text { day step count }= \pm 1801(37.8) \\
\text { Medium rate steps }= \pm 836(87.1 \%) \\
\text { High rate steps }= \pm 1750(153 \%) \\
\text { Low rate steps }= \pm 1643(63.6 \%)\end{array}$ & \\
\hline $\begin{array}{l}\text { Mudge } \\
2010^{170}\end{array}$ & ME & $\mathrm{F}, \mathrm{I}$ & $\begin{array}{l}\mathrm{N}=15 \text {, healthy adults, (full sample } \mathrm{n}=30 \text { ) } \\
27.7(8.9), 50 \% \mathrm{f}\end{array}$ & $\begin{array}{l}\text { Home/community; } 3 \text { days free- } \\
\text { living activity }\end{array}$ & $\begin{array}{l}\text { Time interval: } 1 \text { week (same 3- } \\
\text { day period) }\end{array}$ & $\begin{array}{l}\text { 95\% LOA (absolute, \%) } \\
\text { Mean steps/day: } 3341(39.1 \%) \\
\text { Medium rate steps: } 2111(53.5 \%) \\
\text { High rate steps: } 2521(122 \%)\end{array}$ & $\mathrm{P}$ \\
\hline Ng $2012^{167}$ & $\begin{array}{l}\text { Resp to } \\
\text { change }\end{array}$ & $\mathrm{F}, \mathrm{I}$ & $\begin{array}{l}\mathrm{N}=20 \text {, chronic obstructive pulmonary } \\
\text { disease patients with functional } \\
\text { limitation, } 73(8.5), 60 \% \mathrm{f}\end{array}$ & $\begin{array}{l}\text { Lab; walking course; self- } \\
\text { selected slow and normal paces } \\
\text { with and without a rollator for } \\
5 \mathrm{~min} \text { each }\end{array}$ & $\begin{array}{l}\text { Step rate at slow and normal } \\
\text { paces and with/out rollator } \\
\text { (speed regulated by audio } \\
\text { signals) }\end{array}$ & $\begin{array}{l}\text { ANOVA: significant effect of walking speed }\left(\mathrm{F}_{1,19}=\right. \\
88.69 ; \mathrm{p}<0.01) \text { on step rate as measured by SAM. } \\
\text { Significant effect of rollator }\left(\mathrm{F}_{1,19}=12.39, \mathrm{p}=0.02\right) \text {. } \\
\text { No interactions between speed and rollator. }\end{array}$ & $\mathrm{P}$ \\
\hline \multicolumn{8}{|c|}{ StepWatch Activity Monitor acceptability studies $(\mathrm{n}=\mathbf{2 4})$} \\
\hline Algase 200377 & Acc & $\mathrm{D}, 0$ & $\begin{array}{l}\mathrm{n}=72 \text {, ambulatory nursing home } \\
\text { residents with dementia, full sample } \\
(\mathrm{n}=178) 85.3 \text { (6.31), } 75.3 \%\end{array}$ & $\begin{array}{l}\text { Wandering activity for } 4 \times 4 \mathrm{hr} \\
\text { periods }\end{array}$ & Wear rates & $\begin{array}{l}29.2 \% \text { wore SAM for all } 4 \text { periods. } 83.3 \% \text { accepted } \\
\text { a device for any period. MMSE and age did not } \\
\text { predict device acceptance. } N=288 \text { periods: } 57.98 \% \\
\text { periods had available data, } 0.69 \% \text { periods had } \\
\text { equipment failure, } 0 \% \text { project/staff problems, } \\
1.48 \% \text { setting issues, } 28.80 \% \text { subject issues, other } \\
=11.0 \% \text {. SAM was added later in study }\end{array}$ & - \\
\hline
\end{tabular}




\begin{tabular}{|c|c|c|c|c|c|c|}
\hline Reference & MPA & Para & $\begin{array}{l}\text { Population (n, descriptor, mean (SD) } \\
\text { age, \%female) }\end{array}$ & Comparator & Statistic and outcome & Qual \\
\hline & & & gender NR & SAM for $4 \mathrm{x} 4 \mathrm{hr}$ period & $\begin{array}{l}\text { Appearance: } 3.50 \text { (0.76), Comfort: } 3.47(0.80) \text {, } \\
\text { Concealment: } 3.74 \text { (0.61), Easy application: } 3.19 \\
\text { (0.98), Ease of cleaning: } 3.25 \text { (0.92), Location: } 3.76 \\
\text { (0.55), Safety: } 3.71(0.59) \text {, Size: } 3.53(0.79) \text {, } \\
\text { Weight } 3.76(0.49) \text {. Rated second most highly on } \\
\text { six scores out of four devices }\end{array}$ & \\
\hline Barak 2014 ${ }^{171}$ & Acc & $\mathrm{F}$ & $\begin{array}{l}\mathrm{n}=408,>18,5-30 \text { days after stroke } \\
\text { without contraindications to exercise, } \\
62.02(12.74), 45.1 \% \mathrm{f}\end{array}$ & $\begin{array}{l}\text { Everyday activities for } 2 \text { days, Wear time } \\
\text { removed for bathing, } \\
\text { showering, swimming or } \\
\text { sleeping }\end{array}$ & $\begin{array}{l}\text { Inferred adherence per day = activity (>2 steps) } \\
\text { within each six hour time period (6am-12pm, 12-6 } \\
\text { and 6-12am) for each day. Day } 1=68.1 \% \\
\text { adherence, Day } 2=60.8 \% \text {, Both = } 52.9 \% \text {, Either } \\
\text { day = 76.0\%. Logistic regression indicated that } \\
\text { older individuals with better balance self-efficacy } \\
\text { and walking endurance were more likely to } \\
\text { adhere to the SAM protocol. Written information } \\
\text { and reminders given. }\end{array}$ & \\
\hline $\begin{array}{l}\text { Bergman } \\
2005^{81}\end{array}$ & Acc & $\mathrm{F}$ & $\begin{array}{l}\mathrm{n}=37,>65 \text { living in independent living } \\
(\mathrm{n}=17) \text {, assisted living }(\mathrm{n}=8) \text { and } \\
\text { nursing home facilities }(\mathrm{n}=12) \text { in } \\
\text { Knoxville, } 85.81(4.16), 70.3 \% \mathrm{f}\end{array}$ & $\begin{array}{l}\text { Everyday activities for } 1 \text { full } \\
\text { weekday, removed only for } \\
\text { bathing }\end{array}$ & $\begin{array}{l}\text { Average wear time }=13.66(1.26) \text { hours. } \\
\text { Retirement homes }(n=17)=12.63(1.43) \text { Assisted } \\
\text { living }(n=8)=13.82(1.26) \text { Nursing home }(n=12)= \\
14.13(0.84) \text {. Reminders and instructions were } \\
\text { provided to participants and staff. }\end{array}$ & \\
\hline
\end{tabular}




\begin{tabular}{|c|c|c|c|c|c|c|}
\hline Reference & MPA & Para & $\begin{array}{l}\text { Population (n, descriptor, mean (SD) } \\
\text { age, \%female) }\end{array}$ & Comparator & Statistic and outcome & Qual \\
\hline & & I & $\begin{array}{l}\mathrm{n}=10 \text { ambulant neurological patients } \\
\text { with impairments from different } \\
\text { pathologies and restricted walking } \\
\text { mobility } 59.4 \text { (13.4), } 50 \% \mathrm{f} \text {. }\end{array}$ & $\begin{array}{l}\text { periods. SAM worn for } \\
24 \mathrm{hr} / \text { day }\end{array}$ & $\begin{array}{l}\text { wearing of the monitor throughout the monitoring } \\
\text { period. This was confirmed by visual inspection of } \\
\text { the data" (No clear definition of non-compliance } \\
\text { or visual inspection) }\end{array}$ & \\
\hline $\begin{array}{l}\text { Cavanaugh } \\
2011^{63}\end{array}$ & Acc & $\begin{array}{l}\text { F, D, } \\
\text { I }\end{array}$ & $\begin{array}{l}\mathrm{n}=21 \text {, ambulatory, community-dwelling, } \\
\text { multiple sclerosis patients, } 57.6(12.7) \text {, } \\
57.1 \% \mathrm{f}\end{array}$ & $\begin{array}{l}\text { Everyday activities for } 7 \text { days } \\
\text { (waking hours only except } \\
\text { bathing, sleeping or swimming) }\end{array}$ & $\begin{array}{l}19(90 \%) \text { completed }>=6 \text { days of recording. Range } \\
=3-7 \text { days }\end{array}$ & - \\
\hline $\begin{array}{l}\text { Cavanaugh } \\
2012^{78}\end{array}$ & Acc & $\begin{array}{l}\mathrm{F}, \mathrm{D}, \\
\mathrm{I}\end{array}$ & $\begin{array}{l}\mathrm{n}=57, \text { Parkinson's disease patients. Of } \\
33 \text { complete data } 67.06 \text { (8.75), } 33.3 \% \mathrm{f}\end{array}$ & $\begin{array}{l}\text { All activity for } 7 \text { days, except } \\
\text { bathing, swimming or } \\
\text { showering, worn on least } \\
\text { affected leg. SAM worn at } \\
\text { baseline and } 12 \text { month follow- } \\
\text { up }\end{array}$ & $\begin{array}{l}57 \text { wore monitors at baseline, } 37 \text { at following year. } \\
\text { Data recording problems (incorrect wear and } \\
\text { computer docking issues) = } 4(10.8 \% \text { ). Mean (SD) } \\
\text { days of wear = } 6.7 \text { (1.1) at baseline ( } \mathrm{n}=57), 6.4 \\
\text { (1.0) at } 1 \text { year ( } \mathrm{n}=33 \text { ). In a few cases, participants } \\
\text { decided to wear the monitor 1-2 additional days. } \\
\text { In a few cases, activity data from a day were } \\
\text { excluded due to minimal activity compared to all } \\
\text { other days }\end{array}$ & - \\
\hline Danks 2014 ${ }^{64}$ & Acc & $\begin{array}{l}\text { F, D, } \\
\text { I }\end{array}$ & $\begin{array}{l}\mathrm{n}=23 \text {, stroke survivors ( }>6 \text { months post } \\
\text { stroke), walking without assistance) } \\
\text { (devices allowed), } \mathrm{n}=16 \text { completers, } 66 \\
\text { (range } 40-78 \text { ), } 19 \% \mathrm{f}\end{array}$ & $\begin{array}{l}\text { SAM on non-paretic leg, worn } \\
\text { for all waking hours (except } \\
\text { bathing and swimming) for } 4 \\
\text { weeks }\end{array}$ & $\begin{array}{l}\text { 2/23 (8.7\%) withdrew due to difficulty attaching } \\
\text { the SAM or consistently wearing it. } 2 / 19 \text { (10.5\%) } \\
\text { did not return SAM with minimum } 3 \text { full } \\
\text { days/week captured activity as per protocol and } \\
\text { one admitted to inflating her baseline step activity. }\end{array}$ & - \\
\hline
\end{tabular}




\begin{tabular}{|c|c|c|c|c|c|c|}
\hline Reference & MPA & Para & $\begin{array}{l}\text { Population (n, descriptor, mean (SD) } \\
\text { age, \%female) }\end{array}$ & Comparator & Statistic and outcome & Qual \\
\hline & & & & & 2 withdrew for reasons unrelated to study & \\
\hline $\begin{array}{l}\text { Franklin } \\
2006^{65}\end{array}$ & Acc & $\mathrm{F}, \mathrm{I}$ & $\begin{array}{l}\mathrm{n}=8 \text {, primary total knee arthroplasty } \\
\text { patients with varying characteristics, } \\
\text { age and gender NR }\end{array}$ & $\begin{array}{l}6 \text { knee exercises during week 3- Wear rates } \\
12 \text { after surgery. SAM worn for } \\
4 \text { continuous days before } \\
\text { surgery and during } \\
\text { postoperative week } 6 \text {. }\end{array}$ & $\begin{array}{l}\text { All patients successfully wore the SAM before and } \\
\text { after surgery. No complaints or problems. SAM } \\
\text { returned by post }\end{array}$ & - \\
\hline Gundle $2014^{79}$ & Acc & $\mathrm{F}$ & $\begin{array}{l}\mathrm{n}=29 \text {, lower extremity sarcoma } \\
\text { (primary or recurrent) patients treated } \\
\text { with limb salvage, 55yrs (range 22-76), } \\
62 \% \mathrm{f}\end{array}$ & $\begin{array}{l}\text { Everyday activities for } 7 \text { days } \quad \text { Wear rates } \\
\text { (waking hours only except } \\
\text { bathing, sleeping or swimming) }\end{array}$ & $\begin{array}{l}\text { Patients wearing SAM upside down, incorrectly } \\
\text { positing or non-wear for }>3 \mathrm{hr} \text { a day were excluded } \\
\text { to give n=29. Mean days of data collection in } \\
\text { included patients = } 12 \text { (3), range 6-16. Non-wear } \\
\text { was not defined and n excluded not reported. }\end{array}$ & - \\
\hline Hiatt $2011^{48}$ & Acc & $\begin{array}{l}\mathrm{F}, \mathrm{D}, \\
\mathrm{I}\end{array}$ & $\begin{array}{l}\mathrm{n}=69 \text { randomised, } 62 \text { analysed; } \\
\text { intermittent claudication >=1 yr, } 67 \text { (SD } \\
\mathrm{NR}), 37.8 \% \mathrm{f}\end{array}$ & $\begin{array}{l}\text { Home-based walking exercise Wear rates } \\
2-3 \text { times per week initially for } \\
\text { 30-50min per session; everyday } \\
\text { activities for >=10hr/day for } \\
\text { seven days at screening, } 3 \text { mo } \\
\text { and } 6 \text { mo }\end{array}$ & $\begin{array}{l}\text { Baseline: } 83.5 \% \text { recorded }>10 \mathrm{hr} \text { ambulatory } \\
\text { activity (unclear how detected), } 6 \mathrm{mo} 63 \% \\
\text { recorded }>10 \mathrm{hr}, 3 \mathrm{mo} \mathrm{NR}\end{array}$ & \\
\hline Kong 2014 66 & Acc & $\begin{array}{l}\text { F, D, } \\
\text { I }\end{array}$ & $\begin{array}{l}n=46 \text { (37 completed), inactive obese or } \\
\text { overweight pregnant women, } 26.95 \text { (SD } \\
\text { NR), } 100 \% \text { f }\end{array}$ & $\begin{array}{l}\text { Walking programme, increasing } \\
\text { from } 50-150 \mathrm{~min} / \text { week or no } \\
\text { intervention. SAM worn for } 4 \mathrm{x} \\
1 \text { week periods }\end{array}$ & $\begin{array}{l}>3 \text { days of valid data: timepoint } 1 \mathrm{n}=31 / 37(84 \%) \text {, } \\
\text { timepoint } 2 \mathrm{n}=36 / 37 \text { (97\%), timepoint } 3 \mathrm{n}=35 / 37 \\
(95 \%) \text {, timepoint } 4 \mathrm{n}=35 / 37 \text { (95\%). Exclusions } \\
\text { were mainly due to missing data and SAM }\end{array}$ & - \\
\hline
\end{tabular}




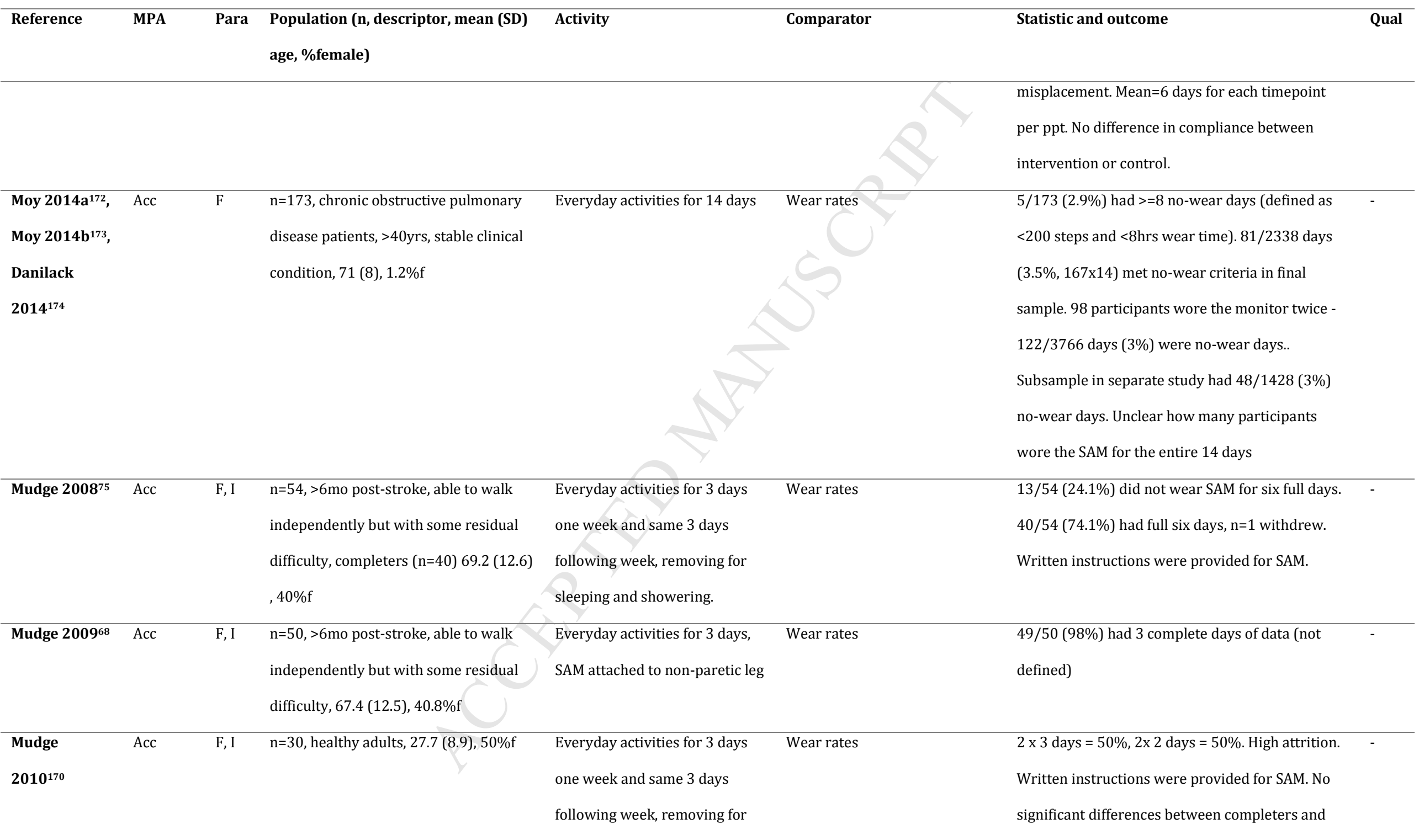




\begin{tabular}{|c|c|c|c|c|c|c|}
\hline Reference & MPA & Para & $\begin{array}{l}\text { Population (n, descriptor, mean (SD) } \\
\text { age, \%female) }\end{array}$ & Comparator & Statistic and outcome & Qual \\
\hline & & & & sleeping and showering. & non-completers & \\
\hline $\begin{array}{l}\text { Nguyen } \\
2011^{69}\end{array}$ & Acc & $\begin{array}{l}\text { F, D, } \\
\text { I }\end{array}$ & $\begin{array}{l}\mathrm{n}=17 \text {, COPD patients who had } \\
\text { completed pulmonary rehabilitation, } 68 \\
(11), 64.7 \% \mathrm{f}\end{array}$ & $\begin{array}{l}\text { Everyday activity (waking } \\
\text { hours only) for } 14 \text { days at } \\
\text { baseline, } 3 \text { mo and } 6 \text { mo ( } 42 \\
\text { days total) }\end{array}$ & $\begin{array}{l}564 \text { person-days of free-living ambulatory activity } \\
\text { were recorded. } 33.2 \text { (9.9) valid days ( }>=10 \mathrm{hrs} \mathrm{of} \\
\text { monitor wear) per person. Mean = } 13.9(0.3) \\
\text { waking hours recorded. } 39 \% \text { had } 14 \text { days at each } \\
\text { timepoint. } 89 \% \text { had } 11 \text { days for each timepoint. }\end{array}$ & \\
\hline $\begin{array}{l}\text { Nguyen } \\
2011^{69}\end{array}$ & Acc & $\begin{array}{l}\text { F, D, } \\
\text { I }\end{array}$ & $\begin{array}{l}\mathrm{n}=60 \text { healthy older adults aged } 60- \\
80 \mathrm{yrs}, 70(6), 51.7 \% \mathrm{f}\end{array}$ & Everyday activity for 7 days Wear time & $\begin{array}{l}\text { Average monitoring days per person }=7.0(1.5) \text {, } \\
442 \text { person days total }\end{array}$ & - \\
\hline $\begin{array}{l}\text { Nguyen } \\
2013^{80}\end{array}$ & Acc & $\mathrm{F}$ & $\begin{array}{l}\mathrm{n}=148 \text {, chronic obstructive pulmonary } \\
\text { disease patients, } 66,5 \text { (8.8), } 22 \% \mathrm{f}\end{array}$ & $\begin{array}{l}\text { Everyday activities for } 7 \text { days Wear time } \\
\text { (waking hours only) }\end{array}$ & $\begin{array}{l}\text { Median wear time }=7 \text { days. Valid day }>=10 \mathrm{hr} \\
(600 \mathrm{~min}) \text { monitor wear. High anxiety symptoms } \\
\text { mean }=874 \text { mins } / \text { day wear, low anxiety } \\
\text { symptoms mean }=899 \mathrm{~min} / \text { day wear }(\mathrm{p}=0.29)\end{array}$ & - \\
\hline Parker $2010^{70}$ & Acc & $\begin{array}{l}\text { F, D, } \\
\text { I }\end{array}$ & $\begin{array}{l}\mathrm{n}=27,>18 \text {, lower limb prosthesis for } \\
>1 \mathrm{yr} \text {. Full sample ( } \mathrm{n}=52 \text {, SAM } \\
\text { subsample) were age } 55.2 \text { (15.8), } \\
21.2 \% \text { f. }\end{array}$ & Everyday activities for 7 days & $\begin{array}{l}\text { Non-wear (not defined): } 4 \text { days }=2 \text { participants } \\
(7.4 \%), 6 \text { days }=3 \text { participants }(11.1 \%)\end{array}$ & - \\
\hline Roos 201271 & Acc & $\begin{array}{l}\text { F, D, } \\
\text { I }\end{array}$ & $\begin{array}{l}\mathrm{n}=54 \text { stroke survivors able to walk } \\
\text { without assistance from another person, } \\
63.7 \text { (10.4), gender NR; } \mathrm{n}=18 \text { retired or } \\
\text { semi-retired older adults living in the } \\
\text { community without walking deficits, }\end{array}$ & $\begin{array}{l}\text { Everyday activity for } 3 \text { days } \\
\text { (waking hours only except } \\
\text { bathing and swimming) }\end{array}$ & $\begin{array}{l}\text { 7/72 (9.7\%) did not have } 3 \text { days of ambulation } \\
\text { activity }(3 / 54(5.6 \%) \text { stroke survivors, } 4 / 18 \\
(22.2 \%) \text { older adults) }\end{array}$ & - \\
\hline
\end{tabular}




\begin{tabular}{|c|c|c|c|c|c|c|c|}
\hline Reference & MPA & Para & $\begin{array}{l}\text { Population (n, descriptor, mean (SD) } \\
\text { age, \%female) }\end{array}$ & Activity & Comparator & Statistic and outcome & Qual \\
\hline & & & 68.9 (6.2), gender NR & & & & \\
\hline $\begin{array}{l}\text { Subramony } \\
2012^{72}\end{array}$ & Acc & $\begin{array}{l}\text { F, D, } \\
\text { I }\end{array}$ & $\begin{array}{l}\mathrm{n}=19 \text {, ambulatory individuals with } \\
\text { different spinocerebellar ataxias, } 56 \\
(10.66), 78.9 \% \mathrm{f}\end{array}$ & $\begin{array}{l}\text { Everyday activities for } 7 \times 24 \mathrm{hr} \\
\text { periods ( } 8 \text { days), day and night }\end{array}$ & Wear rates & $\begin{array}{l}\text { "patients...wore it faithfully through all activities } \\
\text { with no interruptions in the recordings." }\end{array}$ & - \\
\hline Varma $2014^{73}$ & Acc & $\begin{array}{l}\text { F, D, } \\
\text { I }\end{array}$ & $\begin{array}{l}\text { n=195, >=60yrs, } 66.8 \text { (5.6), 76.5\%f. } \\
\text { Obesity (8.3\%), hypertension (71.2\%), } \\
\text { osteoarthritis (61.8\%) and diabetes } \\
\text { (32.6\%) fairly prevalent. }\end{array}$ & Everyday activity for 3-7 days & Wear time & $\begin{array}{l}\text { Average data = } 4.9 \text { days. Average of } 0.8 \text { days } \\
(16.4 \% \text { ) removed from analysis. } 8 / 195 \text { non- } \\
\text { compliant (defined as a) <201 total steps/day, b) } \\
\text { days with <6hr of any activity between wake and } \\
\text { sleep c) days with } 6 \mathrm{hr} \text { of consecutive inactivity (<1 } \\
\text { step) between wake and sleep and d) subjects self- } \\
\text { reported in diary that they hadn't complied). Final } \\
187 \text { participants provided } 4.3 \text { days' data (range 1- } \\
\text { 9) each. Unclear how this subset were chosen } \\
\text { from larger RCT. Vague number of days' wear } \\
\text { prescribed. }\end{array}$ & - \\
\hline White $2012^{175}$ & Acc & $\mathrm{F}$ & $\begin{array}{l}\mathrm{n}=1343, \text { community dwelling adults } \\
>50 \text { with a previous knee injury or } \\
\text { operation, body weight }>\text { median value } \\
\text { for age and sex-specific group, knee } 0 \mathrm{~A} \\
\text { confirmed radiographically, } 63.1(7.8) \text {, } \\
60 \% \mathrm{f}\end{array}$ & $\begin{array}{l}\text { Daily walking for } 7 \text { days, } \\
\text { waking hours only }\end{array}$ & Wear rates & $\begin{array}{l}\text { Out } 1343 \text { eligible participants, } 1116 \text { (83\%) } \\
\text { received a SAM and } 1018 \text { (93\%) wore it for 3+ } \\
\text { days. Of the } 229 \text { who did not receive a SAM, 72\% } \\
\text { refused, } 16 \% \text { had impairments preventing use, } 7 \% \\
\text { had no device available to them, and 5\% had other } \\
\text { reasons. Unclear why high refusal rate or why no }\end{array}$ & - \\
\hline
\end{tabular}




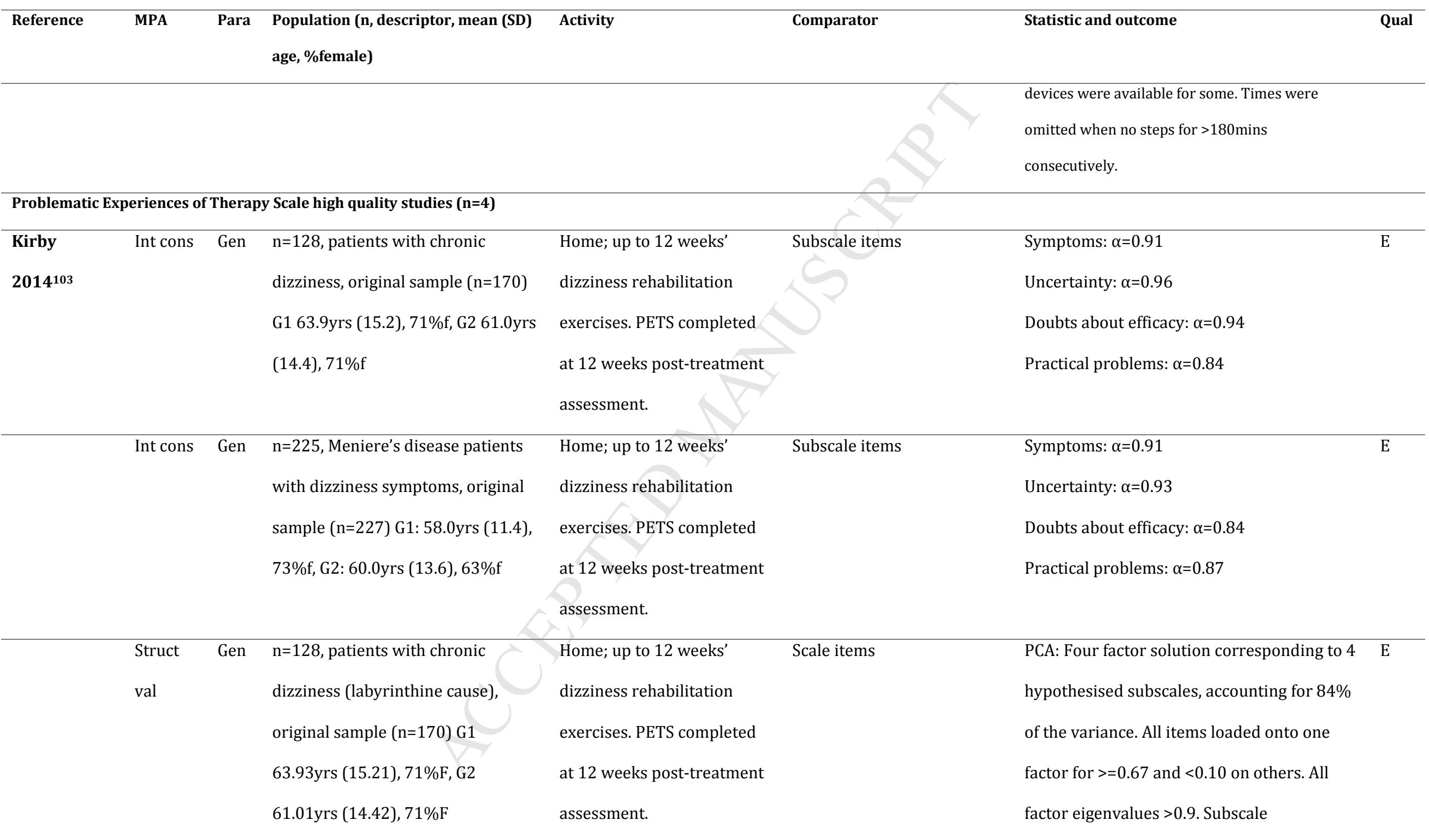




\begin{tabular}{|c|c|c|c|c|c|c|c|}
\hline Reference & MPA & Para & $\begin{array}{l}\text { Population (n, descriptor, mean (SD) } \\
\text { age, \%female) }\end{array}$ & Activity & Comparator & Statistic and outcome & Qual \\
\hline & $\begin{array}{l}\text { Struct } \\
\text { val }\end{array}$ & Gen & $\begin{array}{l}\mathrm{n}=225 \text {, Meniere's disease patients } \\
\text { with dizziness symptoms, original } \\
\text { sample ( } \mathrm{n}=227 \text { ) G1: } 58.0 \mathrm{yrs}(11.4 \text { ), } \\
72.5 \% \mathrm{f}, \mathrm{G} 2: 60.0 \mathrm{yrs}(13.6), 62.5 \% \mathrm{f}\end{array}$ & $\begin{array}{l}\text { Home; up to } 12 \text { weeks' } \\
\text { dizziness rehabilitation } \\
\text { exercises. PETS completed } \\
\text { at } 12 \text { weeks post-treatment } \\
\text { assessment. }\end{array}$ & Scale items & $\begin{array}{l}\text { correlations range from }-0.22 \text { to }-0.53 \text {. } \\
\text { PCA: Four factor solution corresponding to } \\
\text { the } 4 \text { hypothesised subscales, accounting for } \\
81 \% \text { of the variance. All factor loadings } \\
>=0.60 \text { and }<0.11 \text { on other factors. All factor } \\
\text { eigenvalues }>1 \text {. Subscale correlations ranged } \\
\text { from } 0.12 \text { to } 0.36 \text {. }\end{array}$ & $\mathrm{E}$ \\
\hline Problematic Ex & eris & Therar & Scale low quality studies $(n=3)$ & & & & \\
\hline $\begin{array}{l}\text { Yardley } \\
2006^{11}\end{array}$ & Crit val & Gen & $\begin{array}{l}\mathrm{N}=223 \text {, Meniere disease, dizziness or } \\
\text { imbalance symptoms over } 12 \text { months, } \\
\text { VR group (full sample } \mathrm{n}=120 \text { ) } 58 \text { (11.4), } \\
73 \% \mathrm{f} \text {, SC group (full sample } \mathrm{n}=120 \text { ) } \\
60.0(13.6), 62.5 \% \mathrm{f}\end{array}$ & $\begin{array}{l}\text { Home/ community; vestibular } \\
\text { rehabilitation or symptom } \\
\text { control } 3 \text { months }\end{array}$ & $\begin{array}{l}\text { Self-reported adherence for } 9 \text { - } \\
12 \text { weeks or until } \\
\text { asymptomatic ( } 2 \text { questions) }\end{array}$ & $\begin{array}{l}\text { T-test: PETS subscales scores significantly higher } \\
\text { in non-adherent group (all } \mathrm{p}<0.01 \text { ) }\end{array}$ & $P$ \\
\hline Kirby $2014^{103}$ & Cons val & Gen & $\begin{array}{l}\mathrm{n}=128 \text {, patients with chronic dizziness } \\
\text { (labyrinthine cause), original sample } \\
(\mathrm{n}=170) \mathrm{G} 1 \mathrm{n}=8363.93(15.21), 71 \% \mathrm{~F} \\
\mathrm{~g}(\mathrm{~N}=87) 61.01(14.42), 71 \% \mathrm{~F}\end{array}$ & $\begin{array}{l}\text { Up to } 12 \text { weeks' dizziness } \\
\text { rehabilitation exercises. PETS } \\
\text { completed at } 12 \text { weeks post- } \\
\text { treatment assessment and } \\
\text { coded into "no barriers" or } \\
\text { "some barriers" for each } \\
\text { subscale. }\end{array}$ & $\begin{array}{l}12 \text { weeks: Participant self- } \\
\text { report adhering for }>9 \text { weeks or } \\
\text { until asymptomatic }\end{array}$ & $\begin{array}{l}\text { Chi-squared between low adherers. Symptoms: } \\
\text { some barriers } 47 \% \text {, no barriers } 14.6 \% \text { ( }<<0.001) \text {. } \\
\text { Uncertainty: some barriers } 51 \% \text {, no barriers } \\
26.4 \% \text { ( }<<0.01) \text {. Doubts: some barriers } 50 \% \text {, no } \\
\text { barriers } 20 \% \text { (p<0.001). Practical problems: some } \\
\text { barriers } 42.9 \% \text {, no barriers } 25 \%(\mathrm{p}<0.05) \text {. }\end{array}$ & $\mathrm{P}$ \\
\hline
\end{tabular}




\begin{tabular}{|c|c|c|c|c|c|c|c|}
\hline Reference & MPA & Para & $\begin{array}{l}\text { Population (n, descriptor, mean (SD) } \\
\text { age, \%female) }\end{array}$ & Activity & Comparator & Statistic and outcome & Qual \\
\hline Kirby 2014103 & Cons val & Gen & $\begin{array}{l}\mathrm{n}=227 \text {, Meniere's disease patients with } \\
\text { dizziness symptoms in the last } 12 \mathrm{mo} \text {, } \\
\text { Original sample G1: } \mathrm{n}=12058.0 \text { (11.4), } \\
72.5 \% \text { f, G2: } \mathrm{n}=12060.0(13.6), 62.5 \% \mathrm{f}\end{array}$ & $\begin{array}{l}\text { Up to } 12 \text { weeks' dizziness } \\
\text { rehabilitation exercises. PETS } \\
\text { completed at } 12 \text { weeks post- } \\
\text { treatment assessment and } \\
\text { coded into "no barriers" or } \\
\text { "some barriers" for each } \\
\text { subscale. }\end{array}$ & $\begin{array}{l}6 \text { mo: self-report adhering after } \\
12 \text { weeks (any duration) }\end{array}$ & $\begin{array}{l}\text { Chi-squared for maintenance. Symptoms: no } \\
\text { barriers } 47.5 \%(\mathrm{p}<0.01) \text {, doubts } 47.5 \%(\mathrm{p}<0.01) \text {. }\end{array}$ & $\mathrm{P}$ \\
\hline \multicolumn{8}{|c|}{ Problematic Experiences of Therapy Scale acceptability studies $(n=1)$} \\
\hline $\begin{array}{l}\text { Yardley } \\
2006^{11}\end{array}$ & & G & $\begin{array}{l}\mathrm{n}=240 \text { ( } 2 \text { intervention groups), } \\
\text { individuals with Meniere's disease } \\
\text { experiencing dizziness or imbalance in } \\
\text { last } 12 \mathrm{mo} \text {; VR group }=58.0(11.4) \text {, } \\
72.5 \% \mathrm{f} \text {; SC group= } 60.0(13.6), 62.5 \% \mathrm{f}\end{array}$ & $\begin{array}{l}\text { Home-based booklet vestibular } \\
\text { rehabilitation (VR; daily } \\
\text { balance training exercises and } \\
\text { how to tailor to symptoms) or } \\
\text { symptom control (SC; } \\
\text { relaxation and breathing } \\
\text { exercises) for } 3 \text { mo }\end{array}$ & Return rates & $\begin{array}{l}225 / 240 \text { ( } 93.8 \% \text { ) completed PETS at } 3 \mathrm{mo} \text {. No } \\
\text { information on individual item rates. PETS was } \\
\text { packaged with other questionnaires. }\end{array}$ & - \\
\hline \multicolumn{8}{|c|}{ Adherence diaries high quality studies $(n=6)$} \\
\hline \multirow[t]{2}{*}{$\begin{array}{l}\text { Wilbur } \\
20011^{106}\end{array}$} & Crit val & F, D & $\begin{array}{l}\mathrm{n}=156 \text {, sedentary African American and } \\
\text { Caucasian women, mean (SD) age NR } \\
\text { (range } 45-65 \mathrm{yrs}), 100 \% \mathrm{f}\end{array}$ & $\begin{array}{l}\text { Home/community; moderate } \\
\text { intensity 24-week walking } \\
\text { program. }\end{array}$ & $\begin{array}{l}\text { Polar Vantage XL heart rate } \\
\text { monitors }\end{array}$ & $\begin{array}{l}\text { Frequency }=+4.33(S D 7.09) \text { walks on log } \\
(r=0.962, p<0.01) \text {, duration }=+5.0(S D 8.08) \text { min } \\
\text { on monitor }(r=0.536, p<0.001)\end{array}$ & E \\
\hline & Crit val & F, D & $\begin{array}{l}\mathrm{n}=139 \text {, sedentary African American and } \\
\text { Caucasian women, mean (SD) age NR }\end{array}$ & $\begin{array}{l}\text { Home/community; moderate } \\
\text { intensity 24-week walking }\end{array}$ & $\begin{array}{l}\text { Change in fitness }\left(\mathrm{VO}_{2 \max } \text { in a }\right. \\
\text { treadmill test) between }\end{array}$ & $\begin{array}{l}\text { Frequency of walks and change in } \mathrm{VO}_{2 \max }=0.270 \\
(\mathrm{p}<0.01) \text {; average duration and change in } \mathrm{VO}_{2 \max }=\end{array}$ & G \\
\hline
\end{tabular}




\begin{tabular}{|c|c|c|c|c|c|c|c|}
\hline Reference & MPA & Para & $\begin{array}{l}\text { Population (n, descriptor, mean (SD) } \\
\text { age, \%female) }\end{array}$ & Activity & Comparator & Statistic and outcome & Qual \\
\hline & & & (range $45-65 y r s$ ), $100 \% f$ & program. & baseline and post-intervention. & $\begin{array}{l}-0.088 \text { (NS). Similar correlations found between } \\
\text { monitor variables and change in } \mathrm{VO}_{2 \max .}\end{array}$ & \\
\hline $\begin{array}{l}\text { Jeffrey } \\
2012^{107}\end{array}$ & Crit val & $\mathrm{F}$ & $\begin{array}{l}\mathrm{n}=135 \text {, adults over } 60 \text { with osteopenia } \\
\text { of the hip or spine, age } 82.3 \mathrm{yrs}(7.1) \text {, } \\
67 \% \mathrm{f}\end{array}$ & $\begin{array}{l}\text { Home/community; use of active } \\
\text { or sham vibrating platform for } \\
10 \mathrm{~min} \text { per day for up to } 3 \mathrm{yrs}\end{array}$ & $\begin{array}{l}\text { Radio frequency identification } \\
\text { card system }\end{array}$ & $\begin{array}{l}\text { Mean bias close } 0.02 \text { and narrow LOA (graph } \\
\text { only), ICC }=0.96\end{array}$ & G \\
\hline $\begin{array}{l}\text { Lindseth } \\
2005^{104}\end{array}$ & Crit val & $\mathrm{D}$ & $\begin{array}{l}\mathrm{n}=94 \text {, women within the first } 12 \text { weeks } \\
\text { of pregnancy, } 27 \mathrm{yrs}(4.6), 100 \% \mathrm{f}\end{array}$ & $\begin{array}{l}\text { Home/community; exercise for } \\
3 \text { days at } 14 \text { and } 28 \text { weeks' } \\
\text { gestation }\end{array}$ & $\begin{array}{l}\text { Mean Accu-split Power Stride } \\
\text { pedometer counts per day }\end{array}$ & $\mathrm{r}=0.49(\mathrm{p}<0.02)$ & G \\
\hline \multirow[t]{2}{*}{ Shang 2009105} & Crit val & $\begin{array}{l}\text { F, D, } \\
\text { I }\end{array}$ & $\begin{array}{l}\mathrm{n}=126, \text { newly diagnosed cancer } \\
\text { patients, } 60.2 \mathrm{yrs}(10.6), 61 \% \mathrm{f}\end{array}$ & $\begin{array}{l}\text { Home/community; walking } \\
\text { programme } 5 \text { days per week for } \\
\text { 5-35 weeks }\end{array}$ & $\begin{array}{l}\text { Pedometer steps (brand NR) } \\
\text { worn for whole study } \\
\text { (intervention) or first and last } \\
\text { two weeks of study (control) }\end{array}$ & $\rho=0.42(p<0.001)$ & G \\
\hline & Cons val & $\begin{array}{l}\text { F, D, } \\
\text { I }\end{array}$ & $\begin{array}{l}\mathrm{n}=126 \text {, newly diagnosed cancer } \\
\text { patients, } 60.2 \mathrm{yrs}(10.6), 61 \% \mathrm{f}\end{array}$ & $\begin{array}{l}\text { Home/community; walking } \\
\text { programme } 5 \text { days per week for } \\
\text { 5-35 weeks }\end{array}$ & $\begin{array}{l}\text { Physical Activity Questionnaire } \\
\text { METs of previous } 4 \text { weeks } \\
\text { (administered at end of study) }\end{array}$ & $\rho=0.67(\mathrm{p}<0.001)$ & G \\
\hline \multicolumn{8}{|c|}{ Adherence diary low quality studies $(n=30)$} \\
\hline $\begin{array}{l}\text { Castro } \\
2002^{111}\end{array}$ & Crit val & $\begin{array}{l}\text { F, D, } \\
0\end{array}$ & $\begin{array}{l}\mathrm{n}=9 \text {, sedentary and healthy post- } \\
\text { menopausal women providing unpaid } \\
\text { care to a relative with dementia, total } \\
\text { sample }(\mathrm{n}=51) 62.2(9.3), 100 \% \mathrm{f}\end{array}$ & $\begin{array}{l}\text { Hone; exercise programme of } \\
\text { increasing intensity - } 4 \times 30 \text { - } \\
40 \mathrm{mins} \text { per week for } 12 \mathrm{mo} \text {. }\end{array}$ & $\begin{array}{l}\text { Solid-state two-channel } \\
\text { portable microprocessor } \\
\text { recording heart and body } \\
\text { movement for one } 3 \text { day period }\end{array}$ & $\begin{array}{l}87.5 \% \text { agreement between continuous bouts of } \\
\text { physical activity at moderate intensity heart rate } \\
\text { as recorded by the monitor and logs. }\end{array}$ & $\mathrm{P}$ \\
\hline Dougherty & Crit val & D & $\mathrm{n}=77$, single or dual chamber & Home; 8 week home-based & Fitness (peak VO2 measured by & Test unclear, participants achieving $>=80 \%$ & $\mathrm{~F}$ \\
\hline
\end{tabular}




\begin{tabular}{|c|c|c|c|c|c|c|c|}
\hline Reference & MPA & Para & $\begin{array}{l}\text { Population (n, descriptor, mean (SD) } \\
\text { age, \%female) }\end{array}$ & Activity & Comparator & Statistic and outcome & Qual \\
\hline $2015^{123}$ & & & $\begin{array}{l}\text { Implantable Cardioverter Defibrillator } \\
\text { patients taking beta-blockers and } \\
\text { willing to complete the exercise } \\
\text { program, of n=84 at start 56.1(12.1), } \\
20.2 \% \mathrm{f}\end{array}$ & $\begin{array}{l}\text { aerobic training followed by } 16 \\
\text { week maintenance with } \\
\text { increases in heart rate targets }\end{array}$ & $\begin{array}{l}\text { CPET using symptom-limited } \\
\text { treadmill test) }\end{array}$ & $\begin{array}{l}\text { adherence during aerobic conditioning achieved } \\
\text { significantly higher peak VO2 (27.7 (7.0) vs } 24.3 \\
(6.7), p=0.03) \text { and associated exercise outcomes } \\
\text { (data NR) }\end{array}$ & \\
\hline \multirow[t]{2}{*}{$\begin{array}{l}\text { Franklin } \\
2006^{65}\end{array}$} & Crit val & $\mathrm{F}$ & $\begin{array}{l}\mathrm{n}=8 \text {, total knee arthroplasty patients, } \\
\text { total sample (n=21) } 69 \text { (SD NR), 67\%f }\end{array}$ & $\begin{array}{l}\text { Home; daily leg exercises for } 9 \\
\text { weeks }\end{array}$ & $\begin{array}{l}\text { SAM-recorded periods of } \\
\text { sustained step activity for } 4\end{array}$ & $\begin{array}{l}\text { All diary-reported exercise sets were recorded as } \\
\text { high activity peaks on the SAM, plus extra }\end{array}$ & $\mathrm{P}$ \\
\hline & Crit val & $\mathrm{F}, \mathrm{O}$ & $\begin{array}{l}n=21 \text {, total knee arthroplasty patients, } \\
69 \text { (SD NR), 67\%f }\end{array}$ & $\begin{array}{l}\text { Home; daily leg exercises for } 9 \\
\text { weeks }\end{array}$ & $\begin{array}{l}\text { Physical composite score of SF- } \\
12\end{array}$ & $\begin{array}{l}\text { Regression: daily repeats in leg exercise and PCS } \\
\text { changes: slope }=0.34(\mathrm{p}=0.10) \text {, knee reflex repeats } \\
\text { and PCS changes: slope }=0.31(\mathrm{p}=0.09)\end{array}$ & $\mathrm{P}$ \\
\hline $\begin{array}{l}\text { Jakicic } \\
1998^{118}\end{array}$ & Crit val & $\mathrm{F}, \mathrm{D}$ & $\begin{array}{l}\mathrm{N}=50 \text {, overweight women, mean \& SD } \\
\text { NR (range } 25-50 \text { ), } 100 \% \mathrm{f}\end{array}$ & $\begin{array}{l}\text { Home/ community; part of } 20 \\
\text { week trial comparing long } \\
\text { (1x20-40min per day) and } \\
\text { short ( } 2-4 \times 10 \text { min per day) } \\
\text { bouts of exercise }\end{array}$ & $\begin{array}{l}\text { Tri-Trac accelerometers ( } 6 \text { days } \\
\text { between randomly allocated } \\
\text { between weeks } 5 \text { and 10) }\end{array}$ & $\begin{array}{l}29(58 \%) \text { under/accurately reported session } \\
\text { frequency, mean difference }-1.5 \pm 2.4 \text { sessions, } \\
88.5 \pm 24.2 \% \text { sessions matched } \\
21(42 \%) \text { over-reported, mean difference } 2.9 \pm 2.3 \\
\text { sessions, } 44.0 \pm 28.1 \% \text { sessions matched } \\
26(52 \%) \text { under/accurately reported mins per } \\
\text { week: mean difference in duration }-42.8 \pm 45.5 \\
\text { mins } \\
24 \text { (48\%) over-reported: mean difference in } \\
\text { duration } 71.5 \pm 78.4 \text { mins }\end{array}$ & $P$ \\
\hline
\end{tabular}




\begin{tabular}{|c|c|c|c|c|c|c|c|}
\hline Reference & MPA & Para & $\begin{array}{l}\text { Population (n, descriptor, mean (SD) } \\
\text { age, \%female) }\end{array}$ & Activity & Comparator & Statistic and outcome & Qual \\
\hline \multirow[t]{6}{*}{$\begin{array}{l}\text { Lyngcoln } \\
2005^{176}\end{array}$} & \multirow[t]{6}{*}{ Crit val } & \multirow[t]{6}{*}{$\mathrm{F}$} & $\begin{array}{l}\mathrm{n}=15 \text {, individuals }=<18 \text { with a distal } \\
\text { radial fracture managed conservatively } \\
\text { in a cast, } 65.1 \text { (11.1), } 93 \% \mathrm{f}\end{array}$ & \multirow[t]{6}{*}{$\begin{array}{l}\text { Home; home-based hand } \\
\text { therapy exercises for six weeks }\end{array}$} & $\begin{array}{l}\text { Functional status scale } \\
\text { (modified Levine } \\
\text { questionnaire) }\end{array}$ & Pearson's $r=0.63(p<0.05)$ & $\mathrm{P}$ \\
\hline & & & & & Jebsen test of hand function & $\begin{array}{l}\text { Spearman's Item 1: } \rho=0.40 \text {, item 2: } \rho=0.51 \text {, item 3: } \\
\rho=0.25 \text {, item 4: } \rho=0.26 \text {, item 5: } \rho=0.54(p<0.05) \text {, } \\
\text { item 6: } \rho=0.39 \text {, item 7: } \rho=0.32\end{array}$ & $\mathrm{P}$ \\
\hline & & & & & & Pearson's r $=0.46$ & $\mathrm{P}$ \\
\hline & & & & & $\begin{array}{l}\text { Hand dynamometer (grip } \\
\text { strength) }\end{array}$ & Pearson's r $=0.41$ & $\mathrm{P}$ \\
\hline & & & & & Pain (VAS) & Pearson's $r=0.54(p<0.05)$ & $\mathrm{P}$ \\
\hline & & & & & all of the above & $\begin{array}{l}\text { Pearson's } r=0.44 \\
\text { Number of exercises performed and all outcome } \\
\text { measures } r=0.29\end{array}$ & $\mathrm{P}$ \\
\hline \multirow[t]{2}{*}{$\begin{array}{l}\text { McAuley } \\
1991^{125}\end{array}$} & \multirow[t]{2}{*}{ Crit val } & \multirow[t]{2}{*}{ F, D } & $\begin{array}{l}\mathrm{n}=48 \text {, sedentary healthy female } \\
\text { university employees, } 39 \text { (SD NR), }\end{array}$ & $\begin{array}{l}\text { Home/community; twice } \\
\text { weekly supervised } 1 \mathrm{hr} \text { exercise }\end{array}$ & $\begin{array}{l}\text { Body weight (calibrated } \\
\text { balance) }\end{array}$ & $\begin{array}{l}\text { MANOVA by participants }>\text { median overall } \\
\text { adherence: } p<0.1\end{array}$ & $\mathrm{P}$ \\
\hline & & & $100 \% \mathrm{f}$ & $\begin{array}{l}\text { classes for eight weeks, plus } \\
\text { home aerobic exercise of } \\
>15 \mathrm{~min}\end{array}$ & $\begin{array}{l}\text { Body fat (three site method of } \\
\text { skinfold thickness) }\end{array}$ & $\begin{array}{l}\text { MANOVA by participants >median overall } \\
\text { adherence: NS }\end{array}$ & $\mathrm{P}$ \\
\hline $\begin{array}{l}\text { Moseley } \\
2006^{120}\end{array}$ & Crit val & $\mathrm{F}$ & $\begin{array}{l}\mathrm{N}=51 \text {, complex regional pain syndrome } \\
\text { type } 1 \text { of one limb diagnosis from their } \\
\text { treating practitioner, full sample } \mathrm{n}=67 \text { : }\end{array}$ & $\begin{array}{l}\text { Home; RCT of overt vs covert } \\
\text { adherence monitoring of } \\
\text { computer-based motor imagery }\end{array}$ & $\begin{array}{l}\text { In-house software recording } \\
\text { performance time and duration. }\end{array}$ & $\begin{array}{l}\text { Overt monitoring ( } \mathrm{n}=24): 5 \% \text { (95\% CI 0.51-9.48) } \\
\text { underestimation. } \\
\text { Covert monitoring ( } \mathrm{n}=27): 10 \% \text { (95\% CI 3.0-16.9) }\end{array}$ & $\mathrm{P}$ \\
\hline
\end{tabular}




\begin{tabular}{|c|c|c|c|c|c|c|c|}
\hline \multirow[t]{2}{*}{ Reference } & \multirow[t]{2}{*}{ MPA } & \multirow[t]{2}{*}{ Para } & \multirow{2}{*}{$\begin{array}{l}\text { Population (n, descriptor, mean (SD) } \\
\text { age, \%female) } \\
32(10), 48 \% \text { f }\end{array}$} & \multirow{2}{*}{$\begin{array}{l}\text { Activity } \\
\text { home exercise programme }\end{array}$} & \multirow[t]{2}{*}{ Comparator } & \multirow{2}{*}{$\begin{array}{l}\text { Statistic and outcome } \\
\text { overestimation. } \\
\text { Longer symptom duration correlated with greater } \\
\text { inaccuracy. }\end{array}$} & \multirow[t]{2}{*}{ Qual } \\
\hline & & & & & & & \\
\hline \multirow[t]{3}{*}{$\begin{array}{l}\text { Sassi- } \\
\text { Dambron } \\
\text { 1994177 }^{177}\end{array}$} & Crit val & F, D & $\begin{array}{l}n=42 \text {, patients with chronic obstructive } \\
\text { pulmonary disease, } 61.4(7.6), 24 \% \mathrm{f}\end{array}$ & $\begin{array}{l}\text { Home/community; initially } 3 \\
\text { short walks increasing to one } \\
\text { long walk up to a goal of at }>30 \\
\text { min per day for } 8 \text { weeks }\end{array}$ & $\begin{array}{l}\text { Maximum exercise tolerance } \\
\text { (Maximum METs, symptom- } \\
\text { limited treadmill test collecting } \\
\text { blood gases) }\end{array}$ & $\begin{array}{l}\text { Pearson's: Total minutes walked } r=0.32(\mathrm{p}<0.05) \text {, } \\
\text { total days walked } r=0.05\end{array}$ & $\mathrm{~F}$ \\
\hline & & & & & $\begin{array}{l}\text { Maximum exercise tolerance } \\
\text { (peak V02, symptom-limited } \\
\text { treadmill test collecting blood } \\
\text { gases) }\end{array}$ & $\begin{array}{l}\text { Total minutes walked } r=0.18 \text {, total days walked } \\
r=0.05\end{array}$ & $\mathrm{~F}$ \\
\hline & & & & & $\begin{array}{l}\text { Endurance time (constant work } \\
\text { treadmill test) }\end{array}$ & $\begin{array}{l}\text { Total minutes walked } r=0.37(p<0.05) \text {, total days } \\
\text { walked } r=0.22\end{array}$ & $\mathrm{~F}$ \\
\hline \multirow[t]{2}{*}{ Shaw $2005^{119}$} & Crit val & $\mathrm{D}$ & $\begin{array}{l}\mathrm{n}=4 \text {, chronic traumatic brain injury }>1 \\
\text { yr prior to participation with relative } \\
\text { hemiparesis, full sample }(\mathrm{n}=22) 39.3 \\
(14.4), 35 \% \mathrm{f}\end{array}$ & $\begin{array}{l}\text { Home; constraint-induced } \\
\text { movement therapy (mitt wear) } \\
\text { and other behavioural } \\
\text { techniques for } 2 \text { weeks }\end{array}$ & $\begin{array}{l}\text { Sensor and timing device sewn } \\
\text { into mitt }\end{array}$ & Median ICC $=0.97$ & $\mathrm{P}$ \\
\hline & Crit val & F, D & $\begin{array}{l}\mathrm{n}=22 \text {, chronic traumatic brain injury }>1 \\
\text { yr prior to participation with relative } \\
\text { hemiparesis, } 39.3(14.4), 35 \% \mathrm{f}\end{array}$ & $\begin{array}{l}\text { Home; constraint-induced } \\
\text { movement therapy (mitt wear) } \\
\text { and other behavioural } \\
\text { techniques for } 2 \text { weeks }\end{array}$ & $\begin{array}{l}\text { Motor Activity Log Quality of } \\
\text { Movement }\end{array}$ & $\begin{array}{l}\text { T-test adherent }(>57 \%) \text { vs non-adherent }=1.8 \mathrm{vs} \\
1.3(\mathrm{p}=0.065) \text {, correlation in less adherent } \\
\text { participants } r=0.68, \text { none among more adherent } \\
\text { participants }\end{array}$ & $\mathrm{P}$ \\
\hline
\end{tabular}




\begin{tabular}{|c|c|c|c|c|c|c|c|}
\hline Reference & MPA & Para & $\begin{array}{l}\text { Population (n, descriptor, mean (SD) } \\
\text { age, \%female) }\end{array}$ & Activity & Comparator & Statistic and outcome & Qual \\
\hline $\begin{array}{l}\text { Wilcox } \\
2004^{178}\end{array}$ & Crit val & F, D & $\begin{array}{l}\mathrm{n}=18,>=65 \mathrm{yrs}, \text { no cardiovascular } \\
\text { disease, stroke or musculoskeletal } \\
\text { problems, active }=<2 \text { twice a week, full } \\
\text { sample (n=103) } 70.2(4.1), 65 \% \mathrm{f}\end{array}$ & $\begin{array}{l}\text { Home; either Fit \& Firm - brisk } \\
\text { walking and weights or } \\
\text { resistance bands or Stretch \& } \\
\text { Flex - home stretches twice per } \\
\text { week, both } 12 \text { mo }\end{array}$ & $\begin{array}{l}\text { Solid-state portable } \\
\text { microprocessor (Vitalog Corp) } \\
\text { recording heart and body } \\
\text { movement for } 3 \text { days }\end{array}$ & $\begin{array}{l}\text { 10/12 (83\%) in Fit \& Firm condition showed } \\
\text { evidence of an exercise bout of }>=20 \text { min on days } \\
\text { they reported engaging in a home exercise session. } \\
\text { Only } 1 / 6 \text { in Stretch \& Flex condition. }\end{array}$ & $\mathrm{P}$ \\
\hline Yuen $2013^{122}$ & Crit val & $\mathrm{F}, \mathrm{D}$ & $\begin{array}{l}\mathrm{N}=11 \text {, sedentary African-American } \\
\text { women with systemic lupus } \\
\text { erythematosus experiencing fatigue, } \\
48.8(14), 100 \% \mathrm{f}\end{array}$ & $\begin{array}{l}\text { Home; WiiFit exercises for } \\
30 \mathrm{~min}, 3 \text { times a week for } 10 \mathrm{wk}\end{array}$ & WiiFit records & $\begin{array}{l}\text { Mean difference ( } 95 \% \text { LOA) session duration: } \\
3.8 \mathrm{~min} \text { ( } 35 \text { to }-27 \text { mins), } 12.7 \% \text { difference. } \\
\text { ICC }=0.40 \text { ( } 95 \% \text { CI } 0.27-0.51) .72 \% \text { sessions } \\
\text { matched between methods. }\end{array}$ & $\mathrm{P}$ \\
\hline $\begin{array}{l}\text { Aurilio } \\
2000^{126}\end{array}$ & Cons val & $\begin{array}{l}\text { F, D, } \\
0\end{array}$ & $\begin{array}{l}\mathrm{n}=30 \text {, sedentary healthy women aged } \\
30-50 \mathrm{yrs}, 41(6.3) 100 \% \mathrm{f}\end{array}$ & $\begin{array}{l}\text { Home/community; } 12 \text { week } \\
\text { walking programme }\end{array}$ & $\begin{array}{l}\text { Behavioral Risk Factor } \\
\text { Surveillance System Exercise } \\
\text { questionnaire (telephone } \\
\text { interview) }\end{array}$ & $\begin{array}{l}\text { Days walked per week ICC }=0.77(\mathrm{p}=0.01) \\
\text { Spearman's } \rho=0.62(\mathrm{p}<0.01) \\
\text { Mins walked per week ICC }=0.08(\mathrm{p}=0.34), \rho=0.54 \\
(\mathrm{p}<0.01) \\
\text { Miles walked per week ICC }=0.04(\mathrm{P}=0.43), \rho=0.63 \\
(\mathrm{p}<0.01)\end{array}$ & $\mathrm{F}$ \\
\hline Henry 1999179 & Cons val & $\mathrm{F}$ & $\begin{array}{l}\mathrm{N}=15 \text {, healthy adults over } 65,72.8 \text { (SD } \\
\mathrm{NR} \text {, range } 67-82 \text { ), } 73 \% \mathrm{f}\end{array}$ & $\begin{array}{l}\text { Home; } 2,5 \text { or } 8 \text { general } \\
\text { strengthening exercises } 10 \\
\text { times a day }\end{array}$ & $\begin{array}{l}\text { Performance accuracy } \\
\text { assessment tool developed for } \\
\text { study (scored by PTs), inter- } \\
\text { rater reliability } 0.87 \text { for first } \\
\text { exercise and } 0.93 \text { for second }\end{array}$ & Correlations: $\mathrm{r}=0.54$ & $\mathrm{P}$ \\
\hline Khalil 2012 ${ }^{180}$ & Cons val & $\mathrm{F}$ & $\mathrm{n}=15$, mid-stage Huntingdon's disease & Home; PT-prescribed exercises & Intrinsic Motivation Inventory & Spearman's correlations & $\mathrm{P}$ \\
\hline
\end{tabular}




\begin{tabular}{|c|c|c|c|c|c|c|c|}
\hline Reference & MPA & Para & $\begin{array}{l}\text { Population (n, descriptor, mean (SD) } \\
\text { age, \%female) }\end{array}$ & Activity & Comparator & Statistic and outcome & Qual \\
\hline & & & $\begin{array}{l}\text { with difficulties with walking or balance } \\
\text { and stable medical regimen, } 53.6 \text { (range } \\
25-78 \text { ), } 47 \% \mathrm{f}\end{array}$ & $\begin{array}{l}\text { on a DVD at least } 3 \text { times/week } \\
\text { for } 8 \text { weeks }\end{array}$ & $\begin{array}{l}\text { (multidimensional } \\
\text { questionnaire about perceived } \\
\text { interest, enjoyment, } \\
\text { competence, effort, value and } \\
\text { usefulness while performing a } \\
\text { given activity) }\end{array}$ & $\begin{array}{l}\text { Subscales: Interest/enjoyment: } 0.09 \text {, perceived } \\
\text { competence: } 0.39 \text {, effort/importance: } 0.37 \text {, } \\
\text { pressure/tension }-0.63(\mathrm{p}<0.05) \\
\text { value/usefulness: }-0.24\end{array}$ & \\
\hline $\begin{array}{l}\text { McAuley } \\
1991^{125}\end{array}$ & Cons val & $\mathrm{F}, \mathrm{D}$ & $\begin{array}{l}\mathrm{n}=48 \text {, sedentary healthy female } \\
\text { university employees, } 39 \text { (SD NR), }\end{array}$ & $\begin{array}{l}\text { Home/community; twice } \\
\text { weekly supervised } 1 \mathrm{hr} \text { exercise }\end{array}$ & $\begin{array}{l}\text { Self-motivation (self-motivation } \\
\text { inventory) }\end{array}$ & $\begin{array}{l}\text { MANOVA by participants >median overall } \\
\text { adherence: NS }\end{array}$ & $\mathrm{F}$ \\
\hline & & & $100 \% \mathrm{f}$ & $\begin{array}{l}\text { classes for eight weeks, plus } \\
\text { home aerobic exercise of }\end{array}$ & $\begin{array}{l}\text { Self-efficacy (questionnaire of } \\
\text { barriers) }\end{array}$ & $\begin{array}{l}\text { MANOVA by participants }>\text { median overall } \\
\text { adherence: } F(3.43)=3.37, p<0.05\end{array}$ & $\mathrm{~F}$ \\
\hline & & & & $>15 \mathrm{~min}$ & $\begin{array}{l}\text { Post-program perceptions (self- } \\
\text { developed questionnaire of } \\
\text { program success, goal } \\
\text { achievement, improvements in } \\
\text { conditioning and class } \\
\text { enjoyment) }\end{array}$ & $\begin{array}{l}\text { MANOVA by participants >median overall } \\
\text { adherence: NS }\end{array}$ & $\mathrm{P}$ \\
\hline $\begin{array}{l}\text { Wilbur } \\
2005^{181}\end{array}$ & Cons val & F, D & $\begin{array}{l}\mathrm{n}=72 \text {, sedentary healthy, employed } \\
\text { Black and White women } 45-65 \text {, full } \\
\text { sample }(n=90) 49.9(4.8), 100 \% \mathrm{f}\end{array}$ & $\begin{array}{l}\text { Home/community; home-based } \\
\text { moderately intense walking } \\
\text { programme } 4 \text { times per week in } \\
\text { a target heart rate range, } \\
\text { progressing from } 20 \text { to } 30 \mathrm{~min}\end{array}$ & $\begin{array}{l}\text { Exercise recorded by AD in } \\
\text { maintenance phase }\end{array}$ & $\begin{array}{l}\text { Multiple regression: in a model of exercise self- } \\
\text { efficacy, physiological measures, background } \\
\text { characteristics and adherence during intervention } \\
\text { phase, adherence during intervention ( } \mathrm{p}<0.01) \\
\text { and self-efficacy ( } \mathrm{p}=0.02 \text { ) were significant }\end{array}$ & $\mathrm{F}$ \\
\hline
\end{tabular}




\begin{tabular}{|c|c|c|c|c|c|c|c|}
\hline Reference & MPA & Para & $\begin{array}{l}\text { Population (n, descriptor, mean (SD) } \\
\text { age, \%female) }\end{array}$ & Activity & Comparator & Statistic and outcome & Qual \\
\hline & & & & $\begin{array}{l}\text { over } 24 \text { weeks, followed by } 24 \\
\text { weeks maintenance stage }\end{array}$ & & $\begin{array}{l}\text { predictors of walking during maintenance }(40 \% \\
\text { variance explained overall) }\end{array}$ & \\
\hline Steele $2008^{130}$ & $\begin{array}{l}\text { Resp to } \\
\text { change }\end{array}$ & D & $\begin{array}{l}\mathrm{n}=106 \text {, adults }>45 \text { with chronic lung } \\
\text { disease and shortness of breath with } \\
\text { diminished functioning due to a } \\
\text { pulmonary problem who completed } \\
\text { pulmonary rehabilitation, } 67 \text { (SD NR), } \\
\text { 8\%f }\end{array}$ & $\begin{array}{l}\text { Home/community; adherence } \\
\text { to exercise after pulmonary } \\
\text { rehabilitation }\end{array}$ & $\begin{array}{l}\text { Exercise adherence } \\
\text { intervention with weekly phone } \\
\text { calls, } 1 \text { home visit, pedometer } \\
\text { and exercise handbook. }\end{array}$ & $\begin{array}{l}\text { T-test self-reported minutes of activity increased } \\
\text { in adherence intervention group short term: } \\
\text { intervention group } 3(39) \text {, control }-13(26) \\
(p=0.015) \text {. Long term: intervention } 1(45) \text {, control - } \\
8(31)(p=0.335)\end{array}$ & $\mathrm{F}$ \\
\hline $\begin{array}{l}\text { Lindseth } \\
2005^{104}\end{array}$ & TRR & $\mathrm{D}$ & $\begin{array}{l}\mathrm{N}=94 \text {, women within the first } 12 \text { weeks } \\
\text { of pregnancy, } 27(4.6), 100 \% \mathrm{f}\end{array}$ & $\begin{array}{l}\text { Home/community; activity } \\
\text { recorded for three days at } 14 \\
\text { and } 28 \text { weeks' gestation }\end{array}$ & Time interval: 14 weeks & Pearson's correlations: $r=0.61(p<0.01)$ & $\mathrm{F}$ \\
\hline \multicolumn{8}{|c|}{ Adherence diaries acceptability studies (n=19) } \\
\hline Ada $2003^{14}$ & Acc & $\mathrm{U}$ & $\begin{array}{l}\mathrm{n}=14 \text { (control group), stroke survivors } \\
6 \mathrm{mo-5yrs} \text { previously, } 66 \text { (11), 28.6\% } \mathrm{f}\end{array}$ & $\begin{array}{l}\text { Home exercise programme } \\
\text { (strength, balance, } \\
\text { coordination) } 3 \text { times per week } \\
\text { for } 4 \text { weeks }\end{array}$ & Return rate & $\begin{array}{l}\text { Return rate }=8 / 14(57.1 \%) \text { Two subjects had lost } \\
\text { their logs and } 1 \text { subject was lost to follow-up. Logs } \\
\text { returned independently of sessions }\end{array}$ & - \\
\hline $\begin{array}{l}\text { Bauldoff } \\
2001^{113}\end{array}$ & Acc & F, D & $\begin{array}{l}\mathrm{n}=408,>18,5-30 \text { days after stroke } \\
\text { without contraindications to exercise, } \\
62.02(12.74), 45.1 \% \mathrm{f}\end{array}$ & $\begin{array}{l}\text { Home-based } 8 \text { week walking } \\
\text { programme with or without } \\
\text { music, , 2-5 days per week for } \\
>20 \text { mins }\end{array}$ & Return rates & $\begin{array}{l}100 \% \text { complied with log recording. Logs were } \\
\text { returned every } 4 \text { weeks at appointments. } \\
\text { pedometer-recorded distance walked also } \\
\text { recorded (also completed by all) }\end{array}$ & - \\
\hline
\end{tabular}




\begin{tabular}{|c|c|c|c|c|c|c|}
\hline Reference & MPA & Para & $\begin{array}{l}\text { Population (n, descriptor, mean (SD) } \\
\text { age, \%female) }\end{array}$ & Comparator & Statistic and outcome & Qual \\
\hline 1999112 & & I, 0 & $\begin{array}{l}\text { rehabilitation, aged >=45 (male) or } 55 \\
\text { (female), prior or current coronary } \\
\text { heart disease and risk factors, } 69 \text { (11), } \\
33.3 \% \mathrm{f}\end{array}$ & $\begin{array}{l}\text { prescribed cardiac } \\
\text { rehabilitation exercise } \\
\text { programme }\end{array}$ & $\begin{array}{l}\text { weeks. } n=3(15 \%) \text { stopped mailing log and so } \\
\text { ended study. } 7 \text { others dropped out for other } \\
\text { reasons. Up to } 3 \text { telephone and mail reminders per } \\
\text { diary: additional calls or letters required: } 0: n=19 \text {, } \\
\text { 1: } n=4,2: n=4,3: n=3\end{array}$ & \\
\hline $\begin{array}{l}\text { Castro } \\
2002^{111}\end{array}$ & Acc & $\begin{array}{l}\text { F, D, } \\
\text { I, O }\end{array}$ & $\begin{array}{l}\mathrm{n}=51 \text { (exercise group), sedentary and } \\
\text { healthy post-menopausal women >=50 } \\
\text { living with and providing unpaid care to } \\
\text { a relative with dementia; } 62.16 \text { (9.33), } \\
100 \% \mathrm{f}\end{array}$ & $\begin{array}{l}\text { Exercise programme of } \\
\text { increasing intensity }-4 \times 30- \\
40 \mathrm{mins} \text { per week for } 12 \mathrm{mo}\end{array}$ & $\begin{array}{l}\text { Mean return rate }=8.81 / 12 \text { (SD 4.39). Returned } \\
\text { monthly by mail, with phone contacts to obtain } \\
\text { info if not returned }\end{array}$ & - \\
\hline Dyson $1997^{182}$ & Acc & D, 0 & $\begin{array}{l}\mathrm{n}=93 \text {, participants with increased } \\
\text { fasting plasma glucose (range of } 5.5 \text { to } \\
7.7 \mathrm{mmol}^{*} \mathrm{~L}-1 \text { on two consecutive tests } \\
2 \text { weeks) and >=1 risk factor for } \\
\text { diabetes, Full sample ( } \mathrm{n}=227 \text { ) } 50 \text { (9), } \\
59 \% \mathrm{f}\end{array}$ & $\begin{array}{l}\text { 20-30mins exercise } 2-3 \text { times } \\
\text { per week, increasing to } 5-6 \\
\text { times per week over } 12 \mathrm{mo}\end{array}$ & $\begin{array}{l}\text { Return rate: 51/93 (55\%) returned 3/4 diaries, } 15 \\
(16 \%) \text { returned none. Diaries were collected at } \\
\text { each } 3 \text { mo visit, unclear how many returned diaries } \\
\text { in control group }\end{array}$ & - \\
\hline $\begin{array}{l}\text { Franklin } \\
2006^{65}\end{array}$ & Acc & $\mathrm{F}$ & $\begin{array}{l}\mathrm{n}=31 \text {, primary total knee arthroplasty } \\
\text { patients, 69yrs (SD NR), \%f NR }\end{array}$ & $\begin{array}{l}6 \text { knee exercises during weeks Completion rates } \\
3-12 \text { post-TKA }\end{array}$ & $\begin{array}{l}\text { 3/31 (10\%) returned blank logs. } 21 \text { remaining } \\
\text { participants recorded >=3 days exercise per week. } \\
\text { Log completion consistent over weeks 3-12. } \\
\text { Weekly collection by study coordinator, high } \\
\text { attrition ( } n=7) \text {. }\end{array}$ & - \\
\hline
\end{tabular}




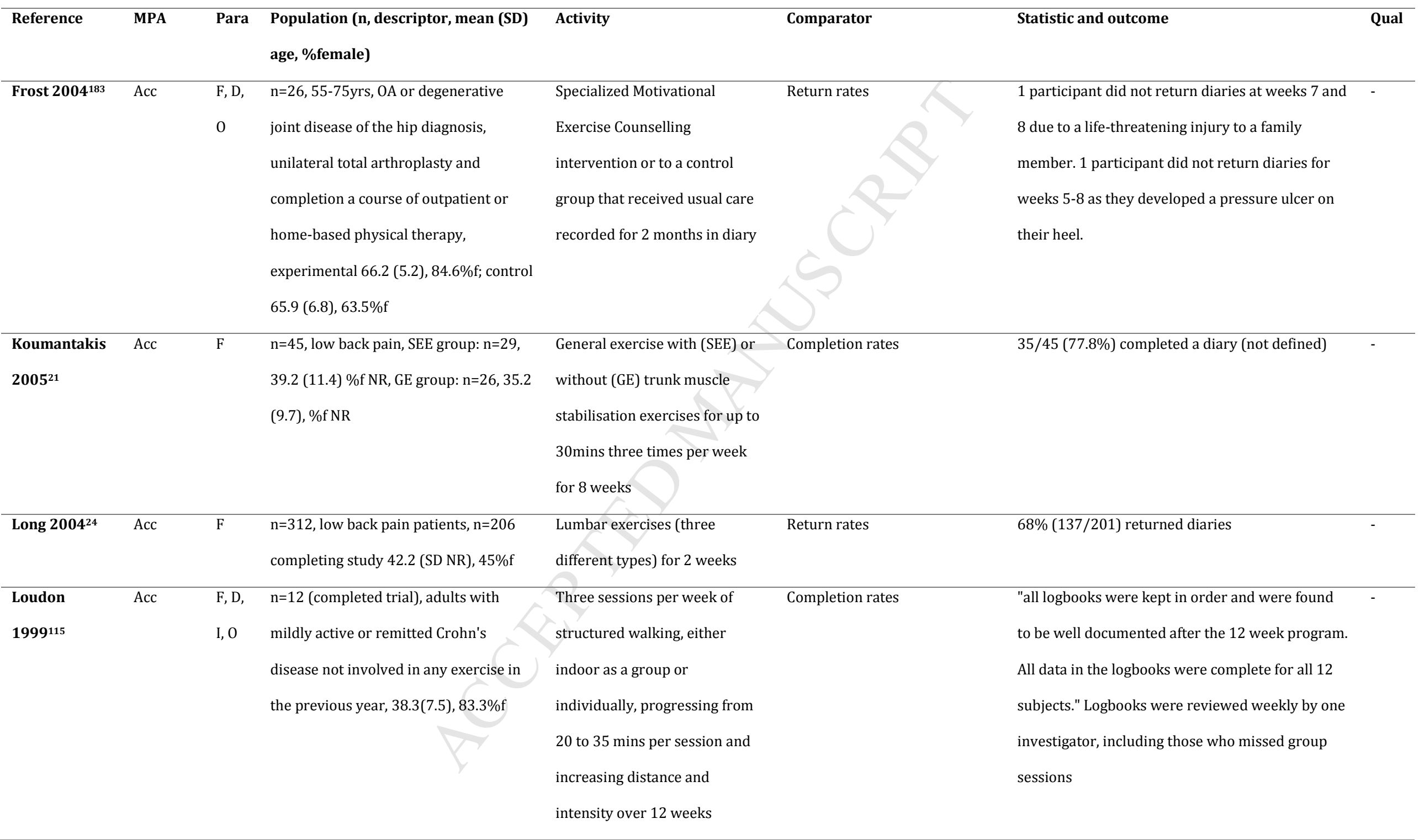




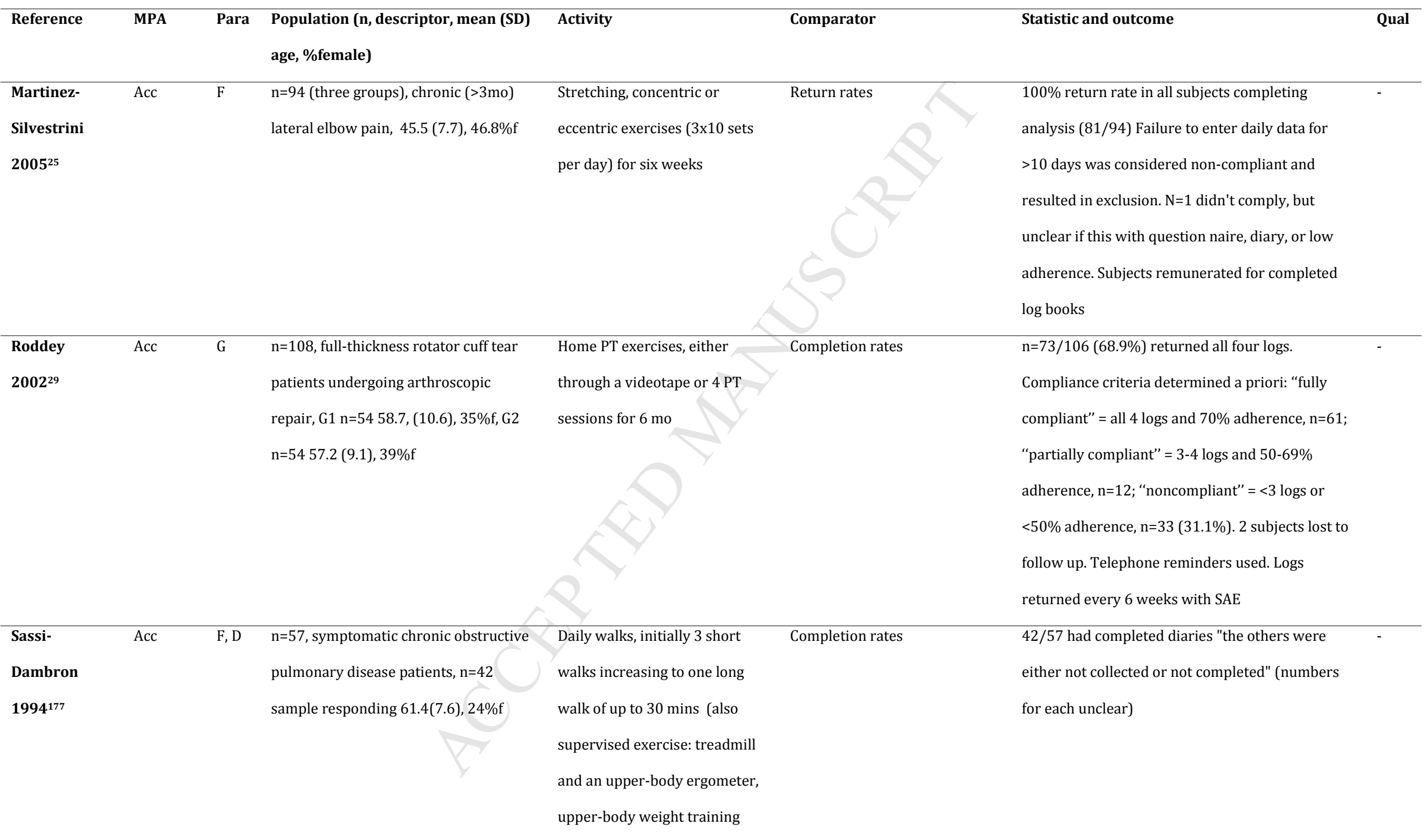




\begin{tabular}{|c|c|c|c|c|c|c|c|}
\hline Reference & MPA & Para & $\begin{array}{l}\text { Population (n, descriptor, mean (SD) } \\
\text { age, \%female) }\end{array}$ & Activity & Comparator & Statistic and outcome & Qual \\
\hline & & & & $\begin{array}{l}\text { over } 8 \text { week block - not } \\
\text { recorded in diary) }\end{array}$ & & & \\
\hline $\begin{array}{l}\text { Schachter } \\
2003^{54}\end{array}$ & Acc & F, D & $\begin{array}{l}\mathrm{n}=143 \text {, sedentary women with } \\
\text { fibromyalgia, groups (1) long bout } \\
\text { exercise (LBE) (n=51) } 41.3 \text { (8.67) } \\
\text { 100\%f; (2) short bouts exercise (SBE) } \\
(\mathrm{n}=56) 41.9(8.57), 100 \% \text { f and (3) } \\
\text { control (no exercise) (n=36) } 42.5 \\
(6.69), 100 \% \text { f }\end{array}$ & $\begin{array}{l}16 \text { week progressive low- } \\
\text { impact aerobics programme } \\
\text { using a videotape, in long or } \\
\text { short bouts }\end{array}$ & Return rates & $\begin{array}{l}45 / 56(80.4 \%) \text { in short bout group, } 42 / 51 \\
(82.4 \%) \text { in long bout group completed the study } \\
\text { and submitted logs ( } 81.3 \% \text { overall) (completion } \\
\text { not defined).= }\end{array}$ & \\
\hline Shang 2009105 & Acc & $\begin{array}{l}\mathrm{F}, \mathrm{D}, \\
\mathrm{I}\end{array}$ & $\begin{array}{l}\mathrm{n}=126, \text { newly diagnosed cancer patients } \\
\text { aged } 21+\text { with no evidence of metastatic } \\
\text { disease, scheduled to receive } \\
\text { chemotherapy or radiotherapy, } 60.2 \\
(10.6), 39 \% \mathrm{f}\end{array}$ & $\begin{array}{l}\text { Individualised home-based } \\
\text { walking and muscle- } \\
\text { strengthening exercise program } \\
5 \text { days per week, throughout } \\
\text { cancer treatment ( } 5-35 \text { weeks). } \\
\text { Control participants continued } \\
\text { their usual physical activity." }\end{array}$ & Completion rates & $\begin{array}{l}17 \text { participants (13.49\%, } 4 \text { (5.9\%) in intervention, } \\
13(22.4 \%) \text { in control) had "significant" missing } \\
\text { data for certain weeks. Missing data were imputed } \\
\text { from telephone logs (correlated highly with } \\
\text { exercise log on other weeks). Logs were mailed } \\
\text { back at the end of each week. Research nurses } \\
\text { would call if logs were not returned on time. }\end{array}$ & \\
\hline $\begin{array}{l}\text { Webb-Peploe } \\
2000^{32}\end{array}$ & Acc & $\begin{array}{l}\text { F, D, } \\
\text { I }\end{array}$ & $\begin{array}{l}\mathrm{n}=24 \text {, patients with ischaemic and } \\
\text { idiopathic dilated cardiomyopathy, } 53 \\
\text { (SD NR), 4.2\%f }\end{array}$ & $\begin{array}{l}\text { Progressive exercises and } \\
20 \text { min bicycle ergometry at } \\
\text { least } 5 \text { days a week for } 8 \text { weeks }\end{array}$ & Completion rates & $\begin{array}{l}\text { 18/24 (75\%) completed diaries, 16/24 (66.7\%) } \\
\text { correctly filled out revolutions pedalled per day. } \\
\text { Unclear if } 18 \text { participants completing diaries were } \\
\text { same as included in final analysis }\end{array}$ & - \\
\hline Williams & Acc & $\mathrm{F}, \mathrm{D}$, & $\mathrm{n}=46$, non-insulin dependent diabetes & Usual exercise over 2 weeks & Return rates & Return rate $=100 \%$ (also fully completed, not & - \\
\hline
\end{tabular}




\begin{tabular}{|c|c|c|c|c|c|c|c|}
\hline Reference & MPA & Para & $\begin{array}{l}\text { Population (n, descriptor, mean (SD) } \\
\text { age, \%female) }\end{array}$ & Activity & Comparator & Statistic and outcome & Qual \\
\hline $1996^{114}$ & & I, 0 & mellitus patients, 60.3 (SD NR), $45.7 \% \mathrm{f}$ & & & defined) & \\
\hline $\begin{array}{l}\text { Wilson } \\
2005^{110}\end{array}$ & Acc & $\begin{array}{l}\mathrm{F}, \mathrm{D}, \\
\mathrm{I}\end{array}$ & $\begin{array}{l}\mathrm{n}=13 \text {, aged } 18-65 \text { who received blood } \\
\text { stem cell or bone marrow transplant } \\
>6 \text { mo prior to participation and low } \\
\text { leisure time physical activity, } \mathrm{n}=17 \text { full } \\
\text { sample, } 48.9 \text { (10.4), } 64.7 \% \mathrm{f}\end{array}$ & $\begin{array}{l}\text { Exercise } 3+\text { times/week for } \\
20 \text { mins continuously in their } \\
\text { HR training zone for } 12 \text { weeks }\end{array}$ & Return rates & $\begin{array}{l}\text { 9/13 (69.2\%) returned completed exercise } \\
\text { diaries. High number of withdrawals and refusals } \\
\text { (76\% intervention acceptability) }\end{array}$ & - \\
\hline Yuen $\mathbf{2 0 1 2}^{33}$ & Acc & F & $\begin{array}{l}\mathrm{n}=26 \text { (intervention group), adults with } \\
\text { systemic sclerosis, } 51.9 \text { (14.3), 80.8\%f }\end{array}$ & $\begin{array}{l}\text { Daily orofacial exercises, teeth } \\
\text { brushing and flossing for } 6 \text { mo }\end{array}$ & Return rates & $\begin{array}{l}\text { Return rate }=11 / 13(84.6 \%) 2 \text { did not return } \\
\text { monthly charts; unclear if all charts were returned } \\
\text { for others. Diaries were posted (SAE provided) } \\
\text { with telephone reminders }\end{array}$ & - \\
\hline \multicolumn{8}{|c|}{ Bassett \& Prapavessis' scale high quality studies (n=3) } \\
\hline Bassett 20112 & Int cons & Gen & $\begin{array}{l}\mathrm{n}=70 \text {, patients with an ankle sprain } \\
\text { undergoing PT, G1 35.9yrs (13.4), G2 } \\
\text { 34.9yrs (12.2), G3 34.9yrs (13.1), 57\%f }\end{array}$ & $\begin{array}{l}\text { Home; PT program with PMT, } \\
\text { attention control or no } \\
\text { information }\end{array}$ & $\begin{array}{l}\text { Cronbach's alpha was } \\
\text { calculated from the means of } \\
\text { participants' mean scores for } \\
\text { each subscale }\end{array}$ & $\alpha=0.63$ & G \\
\hline & Cons val & Gen & $\begin{array}{l}\mathrm{n}=69 \text {, patients with an ankle sprain } \\
\text { undergoing PT, (full sample n=70) G1 } \\
\text { 35.9yrs (13.4), G2 34.9yrs (12.2), G3 } \\
\text { 34.9yrs (13.1), 57\%f }\end{array}$ & $\begin{array}{l}\text { Home; PT program with PMT, } \\
\text { attention control or no } \\
\text { information }\end{array}$ & $\begin{array}{l}\text { Intentions to attend clinic } \\
\text { appointments and to adhere to } \\
\text { home-based therapy ( } 2 \text { items } \\
\text { based on Theory of Planned } \\
\text { Behaviour, } 7 \text { point Likert scale) }\end{array}$ & $\begin{array}{l}\text { Home exercise subscale \& intentions to attend } \\
\text { clinic appointments } r=0.24(p=0.05) \\
\text { Home exercise subscale \& intentions to adhere to } \\
\text { home therapy } r=0.25(p=0.05) \\
\text { Ankle elevation adherence and intentions to } \\
\text { adhere to home therapy } r=0.38(p=0.01)\end{array}$ & G \\
\hline
\end{tabular}




\begin{tabular}{|c|c|c|c|c|c|c|c|}
\hline Reference & MPA & Para & $\begin{array}{l}\text { Population (n, descriptor, mean (SD) } \\
\text { age, \%female) }\end{array}$ & Activity & Comparator & Statistic and outcome & Qual \\
\hline & & & & & & Other variables not correlated & \\
\hline 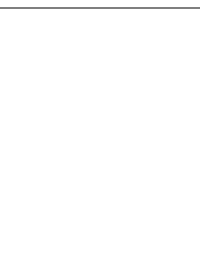 & Crit val & Gen & $\begin{array}{l}\mathrm{n}=69 \text {, patients with an ankle sprain } \\
\text { undergoing PT, (full sample n=70) G1 } \\
\text { 35.9yrs (13.4), G2 34.9yrs (12.2), G3 } \\
\text { 34.9yrs (13.1), 57\%f }\end{array}$ & $\begin{array}{l}\text { Home; PT program with PMT, } \\
\text { attention control or no } \\
\text { information }\end{array}$ & $\begin{array}{l}\text { Ankle function (Lower Limb } \\
\text { Task Questionnaire and Motor } \\
\text { Activity Scale) at the end of the } \\
\text { PT programme. }\end{array}$ & No significant correlations & G \\
\hline Bassett \& Prap & ressis' scal & low qu & lity studies (n=1) & & 8 & & \\
\hline Bassett $2007^{3}$ & Int cons & Gen & $\begin{array}{l}N=47 \text {, diagnosis of acute ankle sprain, } \\
30(12.4), 40 \% \mathrm{f}\end{array}$ & $\begin{array}{l}\text { Clinic; participants randomised } \\
\text { to home-based or clinic-based } \\
\text { three-phase physical therapy } \\
\text { programme }\end{array}$ & $\begin{array}{l}\text { Patients rated their adherence } \\
\text { at the beginning of each clinic } \\
\text { appointment. Means were } \\
\text { calculated for each participant } \\
\text { and subscale }\end{array}$ & Cronbach's $\alpha=0.78$ & $\mathrm{~F}$ \\
\hline Borg 6-20 Rati & of Percei & ed Exer & ion scale high quality studies ( $n=3$ ) & ( & & & \\
\hline Miller $1985^{132}$ & Crit val & I & $\begin{array}{l}\mathrm{n}=113 \text {, healthy adults, } \mathrm{f}=64.8 \mathrm{yrs} \text { (SD } \\
\text { NR) } \mathrm{m}=64.3 \mathrm{yrs}(\mathrm{SD} N R), 52 \% \mathrm{f}\end{array}$ & $\begin{array}{l}\text { Lab; walking on the spot for } \\
2 \text { min at brisk, comfortable pace }\end{array}$ & $\begin{array}{l}\text { Heart rate (Exersentry heart } \\
\text { rate monitor) }\end{array}$ & $\mathrm{r}=0.34 \mathrm{p}=0.0002$ & $\mathrm{E}$ \\
\hline & Crit val & I & $\begin{array}{l}\mathrm{n}=89 \text {, healthy adults, } \mathrm{f}=64.8 \mathrm{yrs} \text { (SD NR) } \\
\mathrm{m}=64.3 \mathrm{yrs} \text { (SD NR), } 52 \% \mathrm{f}\end{array}$ & $\begin{array}{l}\text { Lab; } 600 \mathrm{~m} \text { walk at brisk, } \\
\text { comfortable pace }\end{array}$ & $\begin{array}{l}\text { Heart rate (Exersentry heart } \\
\text { rate monitor). }\end{array}$ & $\mathrm{R}=0.33 \mathrm{p}=0.002$ & G \\
\hline Julius $2012^{133}$ & Cons val & I & $\mathrm{n}=50,65+$ adults with mobility & Lab; 15m walk at self- & Gait speed (GaitMatII) & $\rho=-0.16(p=0.27)$ & G \\
\hline & & & limitations, 76.8yrs (5.5), 66\%f & selected, comfortable pace & $\begin{array}{l}\text { Modified Gait Abnormality } \\
\text { Rating Scale }\end{array}$ & $\rho=0.21(p=0.15)$ & G \\
\hline & & & & & $\begin{array}{l}\text { Energy cost of } 3 \mathrm{~min} \text { treadmill } \\
\text { walk at self-selected pace }\end{array}$ & $\rho=0.01(p=0.95)$ & G \\
\hline
\end{tabular}




\begin{tabular}{|c|c|c|c|c|c|c|c|}
\hline Reference & MPA & Para & $\begin{array}{l}\text { Population (n, descriptor, mean (SD) } \\
\text { age, \%female) }\end{array}$ & Activity & Comparator & Statistic and outcome & Qual \\
\hline & & & & & $\begin{array}{l}\text { (oxygen consumption by open- } \\
\text { circuit spirometry) }\end{array}$ & & \\
\hline & & & & & $\begin{array}{l}\text { Late Life Function and } \\
\text { Disability Questionnaire }\end{array}$ & $\begin{array}{l}\text { Function subscale } \rho=-0.17(p=0.24) \\
\text { basic lower extremity subscale } \rho=-0.20(p=0.17) \text {, } \\
\text { advanced lower extremity subscale } \rho=-0.11 \\
(p=0.47) \text {, disability subscale } \rho=-0.07(p=0.61)\end{array}$ & G \\
\hline & & & & & $\begin{array}{l}\text { Survey of Activities and Fear of } \\
\text { Falling in the Elderly }\end{array}$ & $\begin{array}{l}\text { Fear subscale } \rho=0.26(p=0.07) \text {, activity subscale } \\
\rho=0.13(p=0.35) \text {, restriction subscale } \rho=0.02 \\
(p=0.88)\end{array}$ & G \\
\hline & & & & & $\begin{array}{l}\text { Physical activity during daily } \\
\text { activities (Actigraph } \\
\text { accelerometer) }\end{array}$ & $\rho=0.30(p=0.04)$ & G \\
\hline & & & & & Gait Efficacy Scale & $\rho=-0.33(\mathrm{p}=0.02)$ & G \\
\hline \multicolumn{8}{|c|}{ Borg 6-20 scale low quality studies ( $n=57)$} \\
\hline $\begin{array}{l}\text { Gamberale } \\
1972^{138}\end{array}$ & Crit val & I & $\begin{array}{l}\mathrm{N}=12 \text {, adult healthy men, } 26.5 \text { (SD NR, } \\
\text { range } 20-35), 0 \% \mathrm{f}\end{array}$ & $\begin{array}{l}\text { Lab; randomly assigned } 6 \mathrm{~min} \\
\text { exercise tasks including lifting } \\
\text { weights, pushing a } \\
\text { wheelbarrow and cycling }\end{array}$ & $\begin{array}{l}\text { Heart rate (telemetry, Medenik, } \\
\text { Honeywell) at randomly chosen } \\
\text { values for each workload }\end{array}$ & $\begin{array}{l}\text { Pearson's: Wheelbarrow activity } r=0.42 \text {, lifting } \\
\text { weights } r=0.64 \text {, cycle ergometer } r=0.94\end{array}$ & $\mathrm{P}$ \\
\hline \multirow[t]{2}{*}{ Goslin $1986^{139}$} & Crit val & I & $\begin{array}{l}N=10 \text {, healthy Caucasian males, } 24.3 \\
(2.8), 0 \% \mathrm{f}\end{array}$ & $\begin{array}{l}\text { Lab; treadmill tests with } \\
\text { varying backpack loads and }\end{array}$ & $\begin{array}{l}\text { Heart rate (Hewlett-Packard } \\
\text { telemetry) }\end{array}$ & Correlations: $r=0.47$ & $\mathrm{P}$ \\
\hline & & & & speeds & Oxygen uptake $\left(\mathrm{VO}_{2}\right)$, & $\mathrm{r}=0.75$ & $\mathrm{P}$ \\
\hline
\end{tabular}




\begin{tabular}{|c|c|c|c|c|c|c|c|}
\hline Reference & MPA & Para & $\begin{array}{l}\text { Population (n, descriptor, mean (SD) } \\
\text { age, \%female) }\end{array}$ & Activity & Comparator & Statistic and outcome & Qual \\
\hline & & & & & $\begin{array}{l}\text { Ventilation index (VI) (open } \\
\text { circuit chamber with Beckman } \\
\text { OM-14 and LB-2 oxygen and } \\
\text { carbon dioxide analysers). }\end{array}$ & $\mathrm{r}=0.58$ & $\mathrm{P}$ \\
\hline \multirow[t]{4}{*}{ Goss $2003^{184}$} & Crit val & I & $\begin{array}{l}\mathrm{n}=24, \text { healthy adults, } \mathrm{F}=22.9(5.1), \\
\mathrm{M}=22.4(1.6), 50 \% \mathrm{f}\end{array}$ & $\begin{array}{l}\text { Lab; } 12 \text { 6min exercise trials on } \\
\text { a Nordic Track Total Body } \\
\text { System, with different }\end{array}$ & $\begin{array}{l}\text { Oxygen consumption } \\
\text { (ml/kg/min) - open circuit } \\
\text { spirometry }\end{array}$ & Pearson's r $=0.52$ & $P$ \\
\hline & & & & $\begin{array}{l}\text { combinations of arm and leg } \\
\text { exercises. Three six min }\end{array}$ & $\begin{array}{l}\text { Oxygen consumption (\%V02 } \\
\text { peak) - open circuit spirometry }\end{array}$ & $\mathrm{r}=0.54$ & $P$ \\
\hline & & & & $\begin{array}{l}\text { exercise trials were completed } \\
\text { per session, separate by }>24 \mathrm{hr} \text {. }\end{array}$ & $\begin{array}{l}\text { Respiratory exchange ratio - } \\
\text { open circuit spirometry }\end{array}$ & $\mathrm{r}=0.52$ & $\mathrm{P}$ \\
\hline & & & & & $\begin{array}{l}\text { Heart rate - Eaton Care } \\
\text { Telemetry }\end{array}$ & $\mathrm{r}=0.42$ & $P$ \\
\hline $\begin{array}{l}\text { Lagally } \\
2004^{185}\end{array}$ & Crit val & I & $\begin{array}{l}\mathrm{n}=20,10 \text { novice and } 10 \text { recreationally } \\
\text { trained women, full sample }(\mathrm{n}=28) \\
\text { novice } 21.6(1.5) 100 \% \text { f, recreational } \\
21.9(2.2) 100 \% \mathrm{f}\end{array}$ & $\begin{array}{l}\text { Lab; } 8 \text { repetitions at } 60 \% 1 \mathrm{RM} \text {, } \\
6 \text { repetitions at } 80 \% 1 \mathrm{RM} \text { of a } \\
\text { bench press exercise }\end{array}$ & $\begin{array}{l}\text { Muscle activity using } \\
\text { electromyography (MP100 } \\
\text { EMG system) }\end{array}$ & $\begin{array}{l}\text { No significant correlations (statistics NR) between } \\
\text { RPE and EMG }\end{array}$ & $\mathrm{P}$ \\
\hline $\begin{array}{l}\text { O'Neill } \\
1992^{134}\end{array}$ & Crit val & I & $\begin{array}{l}\mathrm{N}=48 \text {, healthy women with } \\
\text { uncomplicated singleton pregnancies, } \\
30(5), 100 \% \mathrm{f}\end{array}$ & $\begin{array}{l}\text { Location NR; aerobics class at } \\
\text { 13-28 weeks gestation, RPE } \\
\text { estimated at the end of each } \\
\text { exercise track }\end{array}$ & $\begin{array}{l}\text { Heart rate (Polar Sports Tester } \\
\text { PE3000) }\end{array}$ & Pearson's r $=0.27(p>0.05)$ & $\mathrm{F}$ \\
\hline
\end{tabular}




\begin{tabular}{|c|c|c|c|c|c|c|c|}
\hline Reference & MPA & Para & $\begin{array}{l}\text { Population (n, descriptor, mean (SD) } \\
\text { age, \%female) }\end{array}$ & Activity & Comparator & Statistic and outcome & Qual \\
\hline $\begin{array}{l}\text { O'Neill } \\
1992^{134}\end{array}$ & Crit val & I & $\begin{array}{l}\mathrm{N}=11 \text {, healthy women with } \\
\text { uncomplicated singleton pregnancies, } \\
30(3), 100 \% \mathrm{f}\end{array}$ & $\begin{array}{l}\text { Location NR; } 26 \text { min treadmill } \\
\text { exercise at 23-28 and 34-37 } \\
\text { weeks gestation and again at 8+ } \\
\text { weeks after delivery. }\end{array}$ & ECG (Hewlett Packard 1405A) & Pearson's: $r=0.83 p<0.01$ & $\mathrm{P}$ \\
\hline $\begin{array}{l}\text { O'Neill }^{\prime} \\
1992^{134}\end{array}$ & Crit val & I & $\begin{array}{l}\mathrm{N}=12 \text {, healthy women with } \\
\text { uncomplicated singleton pregnancies, } \\
32(4), 100 \% \mathrm{f}\end{array}$ & $\begin{array}{l}\text { Location NR; } 12 \text { min exercise on } \\
\text { a bicycle ergometer at } 34-38 \\
\text { weeks gestation and at } 8+ \\
\text { weeks postpartum }\end{array}$ & ECG (Hewlett Packard 1405A) & Pearson's: $r=0.74, p<0.015$ & $\mathrm{P}$ \\
\hline $\begin{array}{l}\text { O'Neill }^{\prime} \\
1992^{134}\end{array}$ & Crit val & I & $\begin{array}{l}\mathrm{N}=24 \text {, healthy women with } \\
\text { uncomplicated singleton pregnancies, } \\
30(3), 100 \% \mathrm{f}\end{array}$ & $\begin{array}{l}\text { Location NR; } 30 \text { min circuit } \\
\text { training between } 20-28 \text { weeks } \\
\text { gestation }\end{array}$ & $\begin{array}{l}\text { Heart rate (Polar Sports Tester } \\
\text { PE3000) }\end{array}$ & Pearson's $r=0.39 \mathrm{p}>0.05$ & $\mathrm{P}$ \\
\hline $\begin{array}{l}\text { O'Neill }^{\prime} \\
1992^{134}\end{array}$ & Crit val & I & $\begin{array}{l}\mathrm{N}=29 \text {, healthy women with } \\
\text { uncomplicated singleton pregnancies, } \\
31(4), 100 \% \mathrm{f}\end{array}$ & $\begin{array}{l}\text { Location NR; aerobics class at } \\
\text { 29-39 weeks gestation }\end{array}$ & $\begin{array}{l}\text { Heart rate (Polar Sports Tester } \\
\text { PE3000) }\end{array}$ & Pearson's $r=0.35 \mathrm{p}>0.05$ & $\mathrm{P}$ \\
\hline \multirow[t]{2}{*}{$\begin{array}{l}\text { Pandolf } \\
1978^{186}\end{array}$} & Crit val & I & $\begin{array}{l}\mathrm{n}=15 \text {, highly fit males, } 20.2 \text { (SD NR, } \\
\text { range 18-22), } 0 \% \mathrm{f}\end{array}$ & $\begin{array}{l}\text { Lab; climbing and descending a } \\
\text { laddermill and stool stepping at } \\
\text { three different rates, using foot }\end{array}$ & $\begin{array}{l}\text { Heart rate (Sanborn model } 100 \\
\text { Viso Recorder) }\end{array}$ & $\begin{array}{l}\text { Regression: foot over foot climb descent } r=0.56 \text {, } \\
\text { ascent } r=0.74 \text {, both feet to same rung descent } \\
r=0.23 \text {, ascent } r=0.53 \text {, stool stepping } r=0.74\end{array}$ & $\mathrm{P}$ \\
\hline & & & & $\begin{array}{l}\text { over foot climbing and both feet } \\
\text { to same rung climbing, for five } \\
\text { mins each }\end{array}$ & $\begin{array}{l}\text { Oxygen consumption (expired } \\
\text { air and spirometer) }\end{array}$ & $\begin{array}{l}\text { Foot over foot climb descent } r=0.60 \text {, ascent } r=0.72 \text {, } \\
\text { both feet to same rung descent } r=0.45 \text {, ascent } \\
r=0.63 \text {, stool stepping } r=0.82 \text {. }\end{array}$ & $\mathrm{P}$ \\
\hline Pollock & Crit val & I & $\mathrm{n}=13$ healthy adults, $53.5(5.4), 85 \% \mathrm{f}$ & Lab; WiiFit session, including & Heart rate $(30$ s left radial pulse & Pearson's $r=0.32$ ( $p$ value not calculated due to & $\mathrm{P}$ \\
\hline
\end{tabular}




\begin{tabular}{|c|c|c|c|c|c|c|c|}
\hline Reference & MPA & Para & $\begin{array}{l}\text { Population (n, descriptor, mean (SD) } \\
\text { age, \%female) }\end{array}$ & Activity & Comparator & Statistic and outcome & Qual \\
\hline $2013^{135}$ & & & & $\begin{array}{l}5 \mathrm{~min} \text { warm up, exercise from } \\
\text { two WiiFit categories for } 15 \mathrm{~min} \\
\text { each, } 5 \mathrm{~min} \text { cool down, } \\
\text { performed on two days with } \\
\text { different exercises. RPE } \\
\text { assessed during final 30s of } \\
\text { each exercise category. }\end{array}$ & $\begin{array}{l}\text { palpation by experienced study } \\
\text { coordinators) }\end{array}$ & $\begin{array}{l}\text { repeated measures, mixed effects model analysis } \\
\text { found significant association } \mathrm{p}<0.001 \text { ) }\end{array}$ & \\
\hline Row 2012 187 & Crit val & I & $\begin{array}{l}\mathrm{n}=21 \text {, healthy older adults, } 76.6(5.5) \\
43 \% \mathrm{f}\end{array}$ & $\begin{array}{l}\text { Fitness centre; concentric and } \\
\text { eccentric resistance training } \\
\text { using a seated leg press with } \\
50 \% \text { to } 150 \% \text { body weight } \\
\text { loads ( } 4-5 \text { reps), administered } \\
\text { in a random order }\end{array}$ & $\begin{array}{l}\% 1 \mathrm{RM} \text { lifted in a second } \\
\text { session that equated to the } \\
\text { loads lifted in the first session. } \\
\text { Lowest load in each } 10 \% \text { range } \\
\text { and corresponding RPE were } \\
\text { used. }\end{array}$ & $\begin{array}{l}\text { Regression: average RPE for each load strongly } \\
\text { predicted average } \% 1 \mathrm{RM} \text { for each load }\left(\mathrm{R}^{2}=\right. \\
99.5 \%, \mathrm{p}<0.001)\end{array}$ & $\mathrm{P}$ \\
\hline \multirow[t]{3}{*}{$\begin{array}{l}\text { Schaeffer } \\
1995^{136}\end{array}$} & Crit val & I & $\begin{array}{l}\mathrm{N}=16 \text {, healthy women with previous } \\
\text { instructional experience in aerobic } \\
\text { dance, } 23.0(3.7), 100 \% \mathrm{f}\end{array}$ & $\begin{array}{l}\text { Lab; } 8 \text { trials - 1min each for } 8 \\
\text { minutes x } 3 \text { (T1, T2, T3) } \\
\text { including } 4 \text { steps (jumping jack, }\end{array}$ & $\begin{array}{l}\text { Heart rate (CIC Polar heart } \\
\text { monitor) }\end{array}$ & $\begin{array}{l}\text { T1: } r=-0.18, T 2 \text { r=0.01, T3 r=0.26. Partial } \\
\text { correlations controlling for absolute oxygen } \\
\text { consumption: T1: } r=-0.16, \mathrm{~T} 2 \mathrm{r}=0.02 \text {, T3 r=0.25 }\end{array}$ & $\mathrm{P}$ \\
\hline & & & 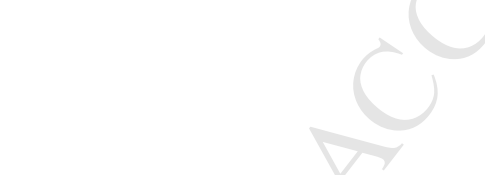 & $\begin{array}{l}\text { power jack, jog and march) at } 2 \\
\text { cadences ( } 124 \text { or } 138 \mathrm{bpm}) \\
\text { along with a leader }\end{array}$ & $\begin{array}{l}\text { Absolute VO2 consumption } \\
\text { (Sensormedics, } 2900 \\
\text { measurement cart) }\end{array}$ & $\mathrm{T} 1: \mathrm{r}=-0.13, \mathrm{~T} 2 \mathrm{r}=-0.01, \mathrm{~T} 3 \mathrm{r}=0.14$ & $\mathrm{P}$ \\
\hline & & & & & $\begin{array}{l}\text { Relative V02 consumption } \\
\text { (Sensormedics, } 2900\end{array}$ & T1: $r=0.25, T 2 r=0.20, T 3 r=-0.02$ & $\mathrm{P}$ \\
\hline
\end{tabular}




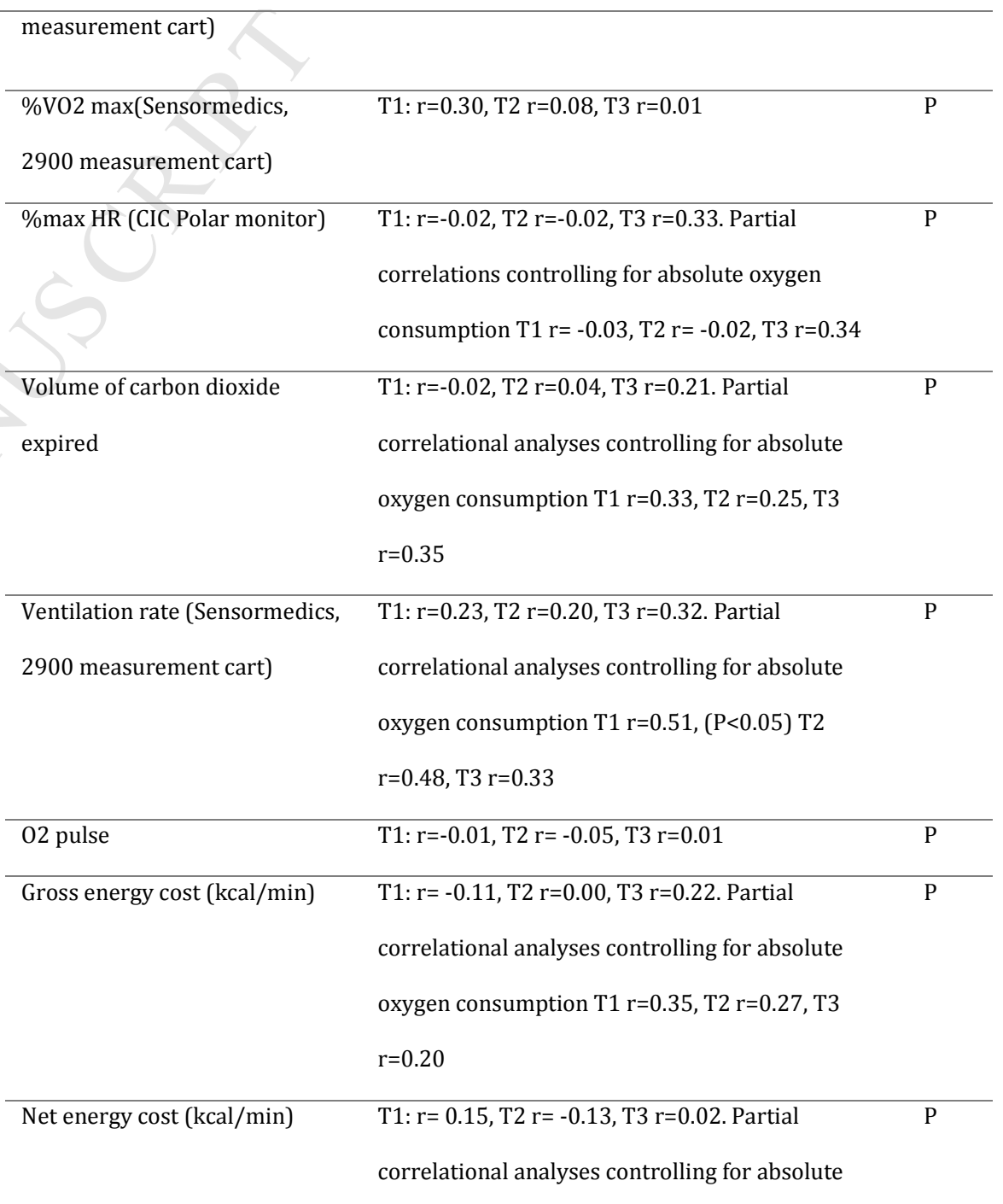




\begin{tabular}{|c|c|c|c|c|c|c|c|}
\hline \multirow[t]{3}{*}{ Reference } & \multirow[t]{3}{*}{ MPA } & \multirow[t]{3}{*}{ Para } & \multirow[t]{3}{*}{$\begin{array}{l}\text { Population (n, descriptor, mean (SD) } \\
\text { age, \%female) }\end{array}$} & \multirow[t]{3}{*}{ Activity } & \multirow[t]{2}{*}{ Comparator } & \multirow{2}{*}{$\begin{array}{l}\text { Statistic and outcome } \\
\text { oxygen consumption } \mathrm{T} 1 \mathrm{r}=0.08, \mathrm{~T} 2 \mathrm{r}=-0.39, \mathrm{~T} 3 \mathrm{r}= \\
-0.39\end{array}$} & \multirow[t]{2}{*}{ Qual } \\
\hline & & & & & & & \\
\hline & & & & & $\begin{array}{l}\text { Respiratory exchange ratio } \\
\text { (Sensormedics, } 2900 \\
\text { measurement cart) }\end{array}$ & $\begin{array}{l}\text { T1: } r=0.37, \mathrm{~T} 2 \mathrm{r}=0.34, \mathrm{~T} 3 \mathrm{r}=0.43 \text {. Partial } \\
\text { correlational analyses controlling for absolute } \\
\text { oxygen consumption } \mathrm{T} 1 \mathrm{r}=0.40, \mathrm{~T} 2 \mathrm{r}=0.34, \mathrm{~T} 3 \\
\mathrm{r}=0.47\end{array}$ & $\mathrm{P}$ \\
\hline $\begin{array}{l}\text { Schaeffer- } \\
\text { Gerschutz }\end{array}$ & Crit val & I & $\begin{array}{l}\mathrm{N}=25 \text {, aerobically trained women, } 21.0 \\
(1.0), 100 \% \mathrm{f}\end{array}$ & $\begin{array}{l}\text { Lab; } 4 \text { combinations of } 3 \mathrm{~min} \\
\text { aerobic steps include dynamic }\end{array}$ & Heart rate (Quinton 4000 ECG) & $\begin{array}{l}\text { Pearson's correlations High impact: } D \mathrm{r}=0.23, \mathrm{~S} \\
\mathrm{r}=0.34 \text {, Low impact } \mathrm{D} r=0.20, \mathrm{~S} r=-0.14\end{array}$ & $P$ \\
\hline \multirow[t]{5}{*}{$2000^{137}$} & & & & $\begin{array}{l}\text { (D) and static (S) high and low } \\
\text { impact arm exercises in a }\end{array}$ & $\begin{array}{l}\text { Percentage maximum heart } \\
\text { rate (Quinton } 4000 \mathrm{ECG} \text { ) }\end{array}$ & $\begin{array}{l}\text { High impact: } \mathrm{D} r=0.27, \mathrm{~S} \mathrm{r}=0.43 \text { (sig } \mathrm{p}<0.03) \text {, Low } \\
\text { impact } \mathrm{D} \mathrm{r}=0.19, \mathrm{~S} r=-0.18\end{array}$ & $P$ \\
\hline & & & & $\begin{array}{l}\text { random order, following } \\
\text { videotaped directions and with } \\
\text { a } 3 \text { min break in between }\end{array}$ & $\begin{array}{l}\text { Relative oxygen consumption } \\
\text { (Sensormedics, } 2900 \\
\text { measurement cart) }\end{array}$ & $\begin{array}{l}\text { High impact: } \mathrm{D} r=-0.07, \mathrm{~S} \mathrm{r}=0.00 \text {, Low impact } \mathrm{D} \\
\mathrm{r}=0.06, \mathrm{~S} r=0.15\end{array}$ & $\mathrm{P}$ \\
\hline & & & & & $\begin{array}{l}\text { Percentage of maximum oxygen } \\
\text { consumption (Sensormedics, } \\
2900 \text { measurement cart) }\end{array}$ & $\begin{array}{l}\text { High impact: } \mathrm{D} r=0.12, \mathrm{~S} r=0.16 \text {, Low impact } \mathrm{D} \\
\mathrm{r}=0.14, \mathrm{~S} r=0.15\end{array}$ & $\mathrm{P}$ \\
\hline & & & & & $\begin{array}{l}\text { Absolute oxygen consumption } \\
\text { (Sensormedics, } 2900 \\
\text { measurement cart) }\end{array}$ & $\begin{array}{l}\text { High impact: } \mathrm{D} r=-0.12, \mathrm{~S} r=-0.20 \text {, Low impact } \mathrm{D} \\
\mathrm{r}=-0.05, \mathrm{~S} r=0.13\end{array}$ & $\mathrm{P}$ \\
\hline & & & & & $\begin{array}{l}\text { Ventilation (Sensormedics, } \\
2900 \text { measurement cart) }\end{array}$ & $\begin{array}{l}\text { High impact: } D \mathrm{r}=0.36 \mathrm{~S} r=0.09 \text {, Low impact } \mathrm{D} \\
\mathrm{r}=0.25, \mathrm{~S} r=0.42(\text { sig } \mathrm{p}<0.03)\end{array}$ & $\mathrm{P}$ \\
\hline
\end{tabular}




\begin{tabular}{|c|c|c|c|c|c|c|c|}
\hline Reference & MPA & Para & $\begin{array}{l}\text { Population (n, descriptor, mean (SD) } \\
\text { age, \%female) }\end{array}$ & Activity & Comparator & Statistic and outcome & Qual \\
\hline & & & & & $\begin{array}{l}\text { Ventilatory equivalent per } \\
\text { oxygen consumption } \\
\text { (Sensormedics, } 2900 \\
\text { measurement cart) }\end{array}$ & $\begin{array}{l}\text { High impact: } \mathrm{D} r=0.62, \mathrm{~S} r=0.40 \text {, Low impact } \mathrm{D} \\
\mathrm{r}=0.39, \mathrm{~S} r=0.45 \text { (all } \mathrm{p}<0.03 \text { ) }\end{array}$ & $\mathrm{P}$ \\
\hline $\begin{array}{l}\text { Stamford } \\
1976^{142}\end{array}$ & Crit val & I & $\begin{array}{l}\mathrm{n}=14 \text {, female undergraduate students, } \\
18.7 \text { (SD NR), } 100 \% \mathrm{f}\end{array}$ & $\begin{array}{l}\text { Lab; } 6 \text { cycling, treadmill } \\
\text { walking, treadmill jogging and } \\
\text { stool stepping tasks performed } \\
\text { at a variety of intensities and } \\
\text { for differing lengths of time in a } \\
\text { randomised order (including } \\
\text { interval tasks). }\end{array}$ & $\begin{array}{l}\text { Identical tasks were performed } \\
\text { with interval RPE rated every } \\
\text { minute of exercise }\end{array}$ & $\begin{array}{l}\text { Pearson's correlations ranged between } 0.71 \text { to } \\
0.90 \text { for all activities }(\mathrm{p}<0.01)\end{array}$ & $\mathrm{P}$ \\
\hline Eng 2002 188 & Cons val & I & $\begin{array}{l}\mathrm{n}=25 \text {, individuals }>1 \text { year post-stroke, } \\
62.6(8.5), 32 \% \mathrm{f}\end{array}$ & $\begin{array}{l}\text { Lab; } 6 \text { minute walk test and } 12 \\
\text { minute walk test, estimation of } \\
\text { exertion at end of each test }\end{array}$ & $\begin{array}{l}\text { Distance walked }(\mathrm{m}) \text {, measured } \\
\text { by amount undertaken on } 42 \mathrm{~m} \\
\text { path }\end{array}$ & Pearson's 6MWT r $=-0.10,12 \mathrm{MWT} \mathrm{r}=-0.06$ & $\mathrm{P}$ \\
\hline \multirow[t]{3}{*}{ Fry $2005^{18}$} & Cons val & I & $\begin{array}{l}\mathrm{n}=12 \text {, adults with MS able to ambulate } \\
\text { for }>6 \mathrm{~min}, 47.3(10.6), 75 \% \mathrm{f}\end{array}$ & $\begin{array}{l}\text { Lab; static standing balance test } \\
\text { Lab; functional stair test }\end{array}$ & $\begin{array}{l}\text { Test scores (best out of 3) } \\
\text { Test scores (best out of 3) }\end{array}$ & $\begin{array}{l}\text { Spearman's } \rho=-0.72(p=0.01) \\
\rho=0.70(p=0.01)\end{array}$ & $\begin{array}{l}\mathrm{P} \\
\mathrm{P}\end{array}$ \\
\hline & & & & Lab; sit-to-stand test & Test scores (best out of 3) & $\rho=0.51(p=0.09)$ & $\mathrm{P}$ \\
\hline & & & & $\begin{array}{l}\text { Lab; 6-minute walk test } \\
\text { (metres) }\end{array}$ & Test score & $\rho=-0.31(\mathrm{p}=0.33)$ & $\mathrm{P}$ \\
\hline $\begin{array}{l}\text { Okhovatian } \\
1997^{145}\end{array}$ & Cons val & I & $\begin{array}{l}\mathrm{n}=10 \text {, able-bodied subjects wearing a } \\
\text { knee-ankle-foot orthosis and using }\end{array}$ & $\begin{array}{l}\text { Location unclear; } 5 \text { min of } \\
\text { walking around looped track at }\end{array}$ & $\begin{array}{l}\text { Speed (calculated by } \\
\text { simultaneously recording time }\end{array}$ & Correlations $r=0.733(p<0.01)$ & $\mathrm{P}$ \\
\hline
\end{tabular}




\begin{tabular}{|c|c|c|c|c|c|c|c|}
\hline Reference & MPA & Para & $\begin{array}{l}\text { Population (n, descriptor, mean (SD) } \\
\text { age, \%female) }\end{array}$ & Activity & Comparator & Statistic and outcome & Qual \\
\hline & & & crutches, 26.7 (SE 1.3), gender NR & $\begin{array}{l}\text { preferred speed, slow speed } \\
\text { and fast speed }\end{array}$ & and distance) & & \\
\hline Hills 2006 ${ }^{146}$ & $\begin{array}{l}\text { Resp to } \\
\text { change }\end{array}$ & I & $\begin{array}{l}n=50, \text { obese }(n=30, \text { age }=47.8(10.8) \\
\text { gender NR) or non-obese }(n=20,36.9 \\
(12.4) \text {, gender NR) sedentary non- } \\
\text { smokers }\end{array}$ & $\begin{array}{l}\text { Grass track; walking on a level } \\
2 \mathrm{~km} \text { once each day for three } \\
\text { days, at "walking for pleasure" } \\
\text { speed for first two days and } \\
\text { maximum pace manageable on } \\
\text { last day }\end{array}$ & $\begin{array}{l}\text { Walking for pleasure speed vs } \\
\text { maximum pace }\end{array}$ & $\begin{array}{l}\text { Mean RPE values significantly higher for both } \\
\text { groups ( } F=133,1, p<0.01 \text { ) }\end{array}$ & $P$ \\
\hline $\begin{array}{l}\text { Kravitz } \\
2003^{147}\end{array}$ & $\begin{array}{l}\text { Resp to } \\
\text { change }\end{array}$ & I & $\begin{array}{l}\mathrm{n}=18 \text {, men and women aged } 20-32 \text { from } \\
\text { boxing exercise classes, } 22.0(2.8), 33 \% \mathrm{f}\end{array}$ & $\begin{array}{l}\text { Lab; } 2 \text { min boxing bouts at } \\
\text { varying tempos }\end{array}$ & $\begin{array}{l}60,72,84,96,108 \text { and } 120 \\
\text { punches per min tempos, } \\
\text { established by a metronome }\end{array}$ & $\begin{array}{l}\text { Friedman non-parametric ANOVA. Significant } \\
\text { differences }(\mathrm{p}<0.05) \text { between RPE ranks }(2.3,2.4 \text {, } \\
2.9,3.2,4.2 \text { for each respective tempo) }\end{array}$ & $\mathrm{P}$ \\
\hline $\begin{array}{l}\text { Lagally } \\
2002^{148}\end{array}$ & $\begin{array}{l}\text { Resp to } \\
\text { change }\end{array}$ & I & $\begin{array}{l}\mathrm{n}=19, \text { healthy adults, } \mathrm{F}=21.8(2.7) \\
\mathrm{M}=23.2(3.6), 47 \% \mathrm{f}\end{array}$ & Lab; 7 resistance exercises & $\begin{array}{l}15 \text { repetitions of } 30 \% 1 \mathrm{RM}, 5 \\
\text { repetitions of } 90 \% 1 \mathrm{RM}\end{array}$ & $\begin{array}{l}\text { ANOVA: All seven exercises showed significantly } \\
\text { higher RPE at higher intensity }(\mathrm{p}<0.01)\end{array}$ & $\mathrm{P}$ \\
\hline $\begin{array}{l}\text { Lagally } \\
2004^{185}\end{array}$ & $\begin{array}{l}\text { Resp to } \\
\text { change }\end{array}$ & I & $\begin{array}{l}\mathrm{n}=28,14 \text { novice and } 14 \text { recreationally } \\
\text { trained women, novice } 21.6(1.5) \\
100 \% \text { f, recreational } 21.9 \text { (2.2) } 100 \% \mathrm{f}\end{array}$ & $\begin{array}{l}\text { Lab; } 8 \text { repetitions at } 60 \% 1 \mathrm{RM} \text {, } \\
6 \text { repetitions at } 80 \% 1 \mathrm{RM} \text { of a } \\
\text { bench press exercise }\end{array}$ & $\begin{array}{l}\text { Increase from } 60 \% 1 \mathrm{Rm} \text { to } \\
80 \% 1 \mathrm{RM}\end{array}$ & $\begin{array}{l}\text { ANOVA - RPE significantly higher (11.29 vs } 13.39 \text {, } \\
\mathrm{p}<0.01) \text { at } 80 \% 1 \mathrm{RM}\end{array}$ & $P$ \\
\hline Leidy $1997^{149}$ & $\begin{array}{l}\text { Resp to } \\
\text { change }\end{array}$ & I & $\mathrm{n}=20$, healthy adults, $35.8(12.4), 80 \% \mathrm{f}$ & $\begin{array}{l}\text { Lab; } 2 \text { mins of: Light activities: } \\
\text { conversing, writing, reading, } \\
\text { playing cards, standing and } \\
\text { waiting; moderate: polishing, } \\
\text { sweeping, dressing, folding }\end{array}$ & $\begin{array}{l}\text { Light, moderate and heavy } \\
\text { activities }\end{array}$ & $\begin{array}{l}\text { Friedman non-parametric ANOVA: RPE varied by } \\
\text { activity intensity in the order hypothesised } \\
(\mathrm{p}<0.001) \text {. Post-hoc tests found significant } \\
\text { differences between light and heavy and heavy } \\
\text { and moderate activity. }\end{array}$ & $\mathrm{P}$ \\
\hline
\end{tabular}


age, \%female)

\begin{tabular}{|c|c|c|c|c|c|c|c|}
\hline & & & & $\begin{array}{l}\text { clothes, level walking; heavy: } \\
\text { stair climbing, hustle walking, } \\
\text { pushing and pulling a vacuum, } \\
\text { carrying groceries, lifting and } \\
\text { moving objects }\end{array}$ & 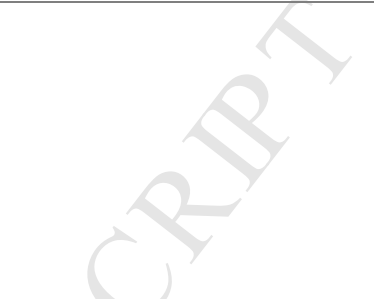 & & \\
\hline $\begin{array}{l}\text { Schaeffer- } \\
\text { Gerschutz } \\
2000^{137}\end{array}$ & $\begin{array}{l}\text { Resp to } \\
\text { change }\end{array}$ & I & $\begin{array}{l}\mathrm{N}=25 \text {, aerobically trained women, } 21.0 \\
(1.0), 100 \% \mathrm{f}\end{array}$ & $\begin{array}{l}\text { Lab; } 4 \text { combinations of } 3 \mathrm{~min} \\
\text { aerobic steps include dynamic } \\
\text { and static high and low impact } \\
\text { arm exercises in a random } \\
\text { order, following videotaped } \\
\text { directions and with a 3min } \\
\text { break in between }\end{array}$ & High vs low impact exercises & $\begin{array}{l}\text { ANOVA: } 10.92 \text { and } 12.16 \text { (high impact) vs } 9.00 \text { and } \\
9.36 \text { (low impact). } \mathrm{F}=34.72(\mathrm{p}<0.03)\end{array}$ & $\mathrm{P}$ \\
\hline $\begin{array}{l}\text { Vasquez } \\
2013^{150}\end{array}$ & $\begin{array}{l}\text { Resp to } \\
\text { change }\end{array}$ & I & $\begin{array}{l}\mathrm{n}=12 \text {, healthy males with }>2 \mathrm{yrs} \\
\text { experience of back squats, } 21.9(1.3) \\
0 \% \mathrm{f}\end{array}$ & $\begin{array}{l}\text { Lab; back squats: } 3 \text { repetitions } \\
\text { of } 50 \% 1 \mathrm{RM} \text { and to volitional } \\
\text { failure, repeated with } 70 \% 1 \mathrm{RM} \\
\text { and } 90 \% 1 \mathrm{RM} \text { in a randomised } \\
\text { order with } 10 \mathrm{~min} \text { rest in } \\
\text { between }\end{array}$ & $\begin{array}{l}\text { Hypothesised differences } \\
\text { between } 3 \text { repetitions at each } \\
\text { intensity but not reps to } \\
\text { volitional failure }\end{array}$ & $\begin{array}{l}\text { ANOVA and one within-subjects factor } \text { - significant } \\
\text { main effect for condition ( } \mathrm{F}=42.8, \mathrm{p}<0.001) \text { and } \\
\text { significant differences between } 3 \text { repetition } \\
\text { intensities }(50=9.5,70=11.7,90=15.3, \mathrm{p}<0.001) \text {. } \\
\text { No sig differences between those to volitional } \\
\text { failure ( } 50=16.7,50=16.5,90=17.4) \text {. }\end{array}$ & $\mathrm{P}$ \\
\hline $\begin{array}{l}\text { Dawes } \\
2005^{189}\end{array}$ & FCV & I & $\begin{array}{l}\mathrm{n}=19 \text {, individuals with acquired brain } \\
\text { injury (age range } 30-60,37 \% \mathrm{f} \text { ), } \mathrm{n}=16 \text {, } \\
\text { individuals with chronic low back pain }\end{array}$ & $\begin{array}{l}\text { Lab; participants asked to } \\
\text { imagine they are cycling up a } \\
\text { progressively steeper hill to a }\end{array}$ & VAS and percentage ratings & $\begin{array}{l}\text { All groups followed an S-shaped curve increase } \\
\text { from nothing to maximum compared to the mean } \\
\text { VAS. Confidence intervals were larger in the centre }\end{array}$ & $\mathrm{P}$ \\
\hline
\end{tabular}




\begin{tabular}{|c|c|c|c|c|c|c|}
\hline \multirow[t]{2}{*}{ Reference } & MPA & Para & $\begin{array}{l}\text { Population (n, descriptor, mean (SD) } \\
\text { age, \%female) }\end{array}$ & Activity & Statistic and outcome & Qual \\
\hline & & & $\begin{array}{l}\text { (age range } 23-55,50 \% \mathrm{f} \text { ), } \mathrm{n}=20 \text { healthy } \\
\text { students (age range } 19-25,50 \% \mathrm{f} \text { ) }\end{array}$ & $\begin{array}{l}\text { point where they are unable to } \\
\text { continue. Each verbal anchor, } \\
\text { administered in a random } \\
\text { order, from the 6-20 was rated } \\
\text { on a } 20 \text {-cm VAS (limits nothing } \\
\text { at all and maximum) and given } \\
\text { a percentage rating. New VAS } \\
\text { and blank cards were given for } \\
\text { each anchor and the previous } \\
\text { one hidden. Participants rated } \\
\text { both breathlessness and leg } \\
\text { fatigue, though as there were no } \\
\text { significant differences only } \\
\text { breathlessness and the VAS } \\
\text { were used in the comparison. }\end{array}$ & $\begin{array}{l}\text { of the scale and significant differences were found } \\
\text { between some anchors but not others, though this } \\
\text { varied between groups. }\end{array}$ & \\
\hline \multirow[t]{3}{*}{ Fry $2005^{18}$} & TRR & I & $\begin{array}{l}\mathrm{n}=12 \text {, adults with MS able to ambulate } \\
\text { for }>6 \mathrm{~min}, 47.3(10.6), 75 \% \mathrm{f}\end{array}$ & $\begin{array}{l}\text { Lab; Static standing balance test } 1 \text { week } \\
\text { (best trial out of } 3 \text { ) }\end{array}$ & Spearman's: $\rho=0.77(p=0.00)$ & $\mathrm{P}$ \\
\hline & & & 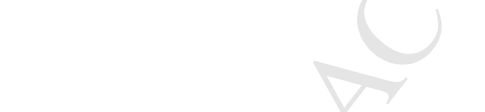 & $\begin{array}{l}\text { Lab; functional stair test (best } \\
\text { trial out of 3) }\end{array}$ & $\rho=0.86(p=0.00)$ & $\mathrm{P}$ \\
\hline & & & & $\begin{array}{l}\text { Lab; sit-to-stand test (best trial } 1 \text { week } \\
\text { out of } 3 \text { ) }\end{array}$ & $\rho=0.70(p=0.01)$ & $\mathrm{P}$ \\
\hline
\end{tabular}




\begin{tabular}{|c|c|c|c|c|c|c|c|}
\hline Reference & MPA & Para & $\begin{array}{l}\text { Population (n, descriptor, mean (SD) } \\
\text { age, \%female) }\end{array}$ & Activity & Comparator & Statistic and outcome & Qual \\
\hline & & & & $\begin{array}{l}\text { 6-minute walk test (rated at } \\
\text { end of each individual test) }\end{array}$ & 1 week & $\rho=0.96(p=0.00)$ & $\mathrm{P}$ \\
\hline Leidy $1997^{149}$ & TRR & I & $\begin{array}{l}n=18 \text {, healthy adults, full sample } \\
(n=20): 35.80 \text { (12.37), 80\%f }\end{array}$ & $\begin{array}{l}\text { Lab; } 2 \text { mins of each activity. } \\
\text { Light activities: conversing, } \\
\text { writing, reading, playing cards, } \\
\text { standing and waiting; } \\
\text { moderate: polishing, sweeping, } \\
\text { dressing, folding clothes, level } \\
\text { walking; heavy: stair climbing, } \\
\text { hustle walking, pushing and } \\
\text { pulling a vacuum, carrying } \\
\text { groceries, lifting and moving } \\
\text { objects }\end{array}$ & $\begin{array}{l}\text { Within } 1 \text { week (mean }=2.8 \\
(1.7))\end{array}$ & $\begin{array}{l}\text { Unclear; no significant differences in RPE (data } \\
\text { NR) }\end{array}$ & $\mathrm{P}$ \\
\hline Row 2012 187 & TRR & I & $\begin{array}{l}\mathrm{n}=21 \text {, healthy older adults, } 76.6(5.5), \\
43 \% \mathrm{f}\end{array}$ & $\begin{array}{l}\text { Fitness centre; concentric and } \\
\text { eccentric resistance training } \\
\text { using a seated leg press with } \\
50 \% \text { to } 150 \% \text { body weight } \\
\text { loads (4-5 repetitions), } \\
\text { administered in a random order }\end{array}$ & $\begin{array}{l}\text { Second presentation of the } \\
\text { same five loads at the end of the } \\
\text { session }\end{array}$ & ICC $=0.729$ & $\mathrm{P}$ \\
\hline $\begin{array}{l}\text { Skatrud- } \\
\text { Mickelson }\end{array}$ & TRR & I & $\begin{array}{l}\mathrm{n}=21 \text {, healthy adults aged } 18-74 \text { of all } \\
\text { BMI classes, full sample } n=117(61.2 \%\end{array}$ & $\begin{array}{l}\text { Lab; } 0.29 \text { mile indoor lap 1) } \\
\text { very slow walk, 2) normal }\end{array}$ & $\begin{array}{l}\text { Mail survey 6-8 weeks later } \\
\text { asking participants to recall the }\end{array}$ & $\begin{array}{l}\text { Wilcoxon signed ranks test: significant difference } \\
\text { between median ranks ( } \mathrm{p}=0.02 \text { ) between times }\end{array}$ & $\mathrm{P}$ \\
\hline
\end{tabular}




\begin{tabular}{|c|c|c|c|c|c|c|c|}
\hline Reference & MPA & Para & $\begin{array}{l}\text { Population (n, descriptor, mean (SD) } \\
\text { age, \%female) }\end{array}$ & Activity & Comparator & Statistic and outcome & Qual \\
\hline $2011^{152}$ & & & aged $18-49,38.8 \%$ aged $50-74) 57 \% f$ & paced walk, 3) brisk walk or jog & RPE for the three laps & & \\
\hline $\begin{array}{l}\text { Stamford } \\
1976^{142}\end{array}$ & TRR & I & $\begin{array}{l}\mathrm{n}=14 \text {, female undergraduate students, } \\
18.7 \text { (SD NR), } 100 \% \mathrm{f}\end{array}$ & $\begin{array}{l}\text { Lab; } 2 \text { x one set of cycle } \\
\text { ergometer tasks including a } \\
\text { range of intensities }\end{array}$ & $\begin{array}{l}\text { Part of } 12 \text { work tasks in a } \\
\text { randomised order over } 4 \\
\text { sessions - could be in the same } \\
\text { session or 2-8 days later }\end{array}$ & Pearson's r $=0.90$ & $P$ \\
\hline Hills 2006 ${ }^{146}$ & ME & I & $\begin{array}{l}n=50 \text {, obese }(n=30 \text {, age }=47.8(10.8) \text {, } \\
\text { gender NR) or non-obese ( } n=20,36.9 \\
(12.4) \text {, gender NR) sedentary non- } \\
\text { smokers }\end{array}$ & $\begin{array}{l}\text { Grass track; walking on a level } \\
2 \mathrm{~km} \text { track once each day for } \\
\text { two days, at "walking for } \\
\text { pleasure" speed }\end{array}$ & & Mean bias $=-0.1, \mathrm{LOA}=2.1$ & $\mathrm{~F}$ \\
\hline
\end{tabular}

Yamax Digiwalker CW series high quality studies (n=1) 


\begin{tabular}{|c|c|c|c|c|c|c|c|}
\hline Reference & MPA & Para & $\begin{array}{l}\text { Population (n, descriptor, mean (SD) } \\
\text { age, \%female) }\end{array}$ & Activity & Comparator & Statistic and outcome & Qual \\
\hline $\begin{array}{l}\text { Kinunnen } \\
2011^{154}\end{array}$ & Crit val & $\mathrm{F}, 0$ & $\begin{array}{l}\mathrm{n}=58 \text {, overweight and obese } \\
\text { pregnant women (BMI>25), median } \\
\text { age } 32 \text { yrs (IQR } 27-36), 100 \% \mathrm{f}\end{array}$ & Home/community; everyday & $\begin{array}{l}\text { GT1M Actigraph (time in } \\
\text { sedentary, light, moderate } \\
\text { and vigorous activity and step } \\
\text { count) }\end{array}$ & $\begin{array}{l}\text { LOA for mean value (6026 steps) = } \\
\text {-2690 to } 2656 \text { steps/day. } \\
\text { Lowest step count } 906 \text { steps (LOA - } 297 \text { to } 4897 \text { ) } \\
\text { Highest } 12018 \text { steps (LOA -4753 to 33) } \\
\text { No effect of BMI and gestational age. } \\
\text { Steps/day Spearman's } \rho=0.78 \text { ( } 0.59-0.90 \text { ) } p<0.001 \\
>=8000 \text { steps/day (CW) and >=30min moderate- } \\
\text { vigorous physical activity per day (GT1M) } \mathrm{k}=0.45 \\
\text { (0.24-0.67), >8000 or }<8000 \text { steps/day (CW \& } \\
\text { GT1M) k=0.63 ( } 0.43 \text { to } 0.83 \text { ) } \\
\text { Wilcoxon signed-rank test for absolute step count } \\
\text { between devices = medians } 5961 \text { vs } 5687 \text { (p=0.37) }\end{array}$ & $\mathrm{G}$ \\
\hline \multicolumn{8}{|c|}{ Yamax Digiwalker CW series low quality studies (n=2) } \\
\hline $\begin{array}{l}\text { Martin } \\
2012^{155}\end{array}$ & Crit val & $\mathrm{F}$ & $\begin{array}{l}\mathrm{n}=18 \text {, community dwelling older adults, } \\
\mathrm{BMI}<30 \text {, able to ambulate without } \\
\text { assistance for }>100 \mathrm{~m}, 63.6 \text { (SD NR), } \\
67 \% \mathrm{f}\end{array}$ & $\begin{array}{l}\text { Lab; walks at 50, } 66 \text { and } 80 \\
\text { steps/min (in time with } \\
\text { metronome) and self-selected } \\
\text { speed on a } 40 \mathrm{~m} \text { indoor track ( } 8 \\
\text { total walks) }\end{array}$ & $\begin{array}{l}\text { Average of } 2 \text { observers with } \\
\text { handheld counters (if within } \\
5 \% \text { steps). } 100 \% \text { agreement for } \\
88 \% \text { trials, no discrepancies } \\
>5 \% \text {. }\end{array}$ & $\begin{array}{l}\text { Percentage error } \\
50 \text { steps } / \mathrm{min}=66.8 \%, 66=40.8 \%, 80=22.7 \% \text { and } \\
\mathrm{SS}=4.8 \%\end{array}$ & $\mathrm{P}$ \\
\hline $\begin{array}{l}\text { Martin } \\
2012^{155}\end{array}$ & $\begin{array}{l}\text { Inter- } \\
\text { rater rel }\end{array}$ & $\mathrm{F}$ & $\begin{array}{l}\mathrm{n}=18 \text {, community dwelling older adults, } \\
\mathrm{BMI}<30 \text {, able to ambulate without } \\
\text { assistance for }>100 \mathrm{~m}, 63.6 \text { (SD NR), }\end{array}$ & $\begin{array}{l}\text { Lab; walks at self-selected } \\
\text { speed on a } 40 \mathrm{~m} \text { indoor track }\end{array}$ & $\begin{array}{l}\text { Three pedometers of the same } \\
\text { brand randomly assigned to a } \\
\text { participant and compared }\end{array}$ & ICC $=0.70(0.20-0.89)$ & $P$ \\
\hline
\end{tabular}


age, \%female)

$67 \%$ f

\section{Yamax Digiwalker CW series acceptability studies (n=1)}

\begin{tabular}{|c|c|c|c|c|c|c|c|}
\hline $\begin{array}{l}\text { Kinunnen } \\
2011^{154}\end{array}$ & Acc & $\mathrm{F}, \mathrm{O}$ & $\begin{array}{l}\mathrm{n}=93 \text {, overweight and obese pregnant } \\
\text { women, median } 13 \text { weeks' gestation, } \\
\text { median age } 32 \text { (IQR 27-36), 100\% f }\end{array}$ & $\begin{array}{l}\text { Steps per day averaged over } 4 \\
\text { days }\end{array}$ & Missing data & $\begin{array}{l}\mathrm{n}=3 \text { women did not have pedometer data (unclear } \\
\text { if out of } 61 \text { with complete accelerometer data } \\
\text { (worn simultaneously) or out of } 93 \text { original } \\
\text { sample). Accelerometer had much higher missing } \\
\text { data. Authors discuss that in some cases step } \\
\text { counts were much lower on pedometer than } \\
\text { accelerometer, suggesting non-wear or a tilt angle } \\
\text { that did not properly detect steps }\end{array}$ & - \\
\hline \multicolumn{8}{|c|}{ Joint Protection Behaviour Assessment low quality studies ( $\mathrm{N}=22)$} \\
\hline \multirow[t]{4}{*}{$\begin{array}{l}\text { Hammond } \\
1999 b^{157}\end{array}$} & \multirow[t]{4}{*}{ Cons val } & \multirow[t]{4}{*}{$\mathrm{A}$} & \multirow{4}{*}{$\begin{array}{l}\mathrm{n}=35 \text {, RA patients with wrist or } \\
\text { metacarophalangeal involvement and } \\
\text { some restriction in ability to perform }\end{array}$} & \multirow{4}{*}{$\begin{array}{l}\text { Home; use of joint protection in } \\
\text { ADLs after a group education } \\
\text { programme }\end{array}$} & $\begin{array}{l}\text { Grip strength (digital } \\
\text { dynamometer) }\end{array}$ & Spearman's $\rho=-0.11$ (NS) & $F$ \\
\hline & & & & & $\begin{array}{l}\text { Hand Joint Alignment and } \\
\text { Motion Sale (ROM and } \\
\text { deformity) }\end{array}$ & $\rho=0.06$ (NS) & $\mathrm{F}$ \\
\hline & & & & & $\begin{array}{l}\text { Frequency of joint protection } \\
\text { practice ( } 7 \text { pt scale } 1=\text { once a } \\
\text { week, } 7=\text { daily) }\end{array}$ & $\begin{array}{l}\rho=0.47 \text { (significant). Also predicted in regression } \\
\operatorname{model}(\beta=5.35, p=0.02)\end{array}$ & $P$ \\
\hline & & & & & Hand pain (VAS) & $\rho=-0.02$ & \\
\hline
\end{tabular}




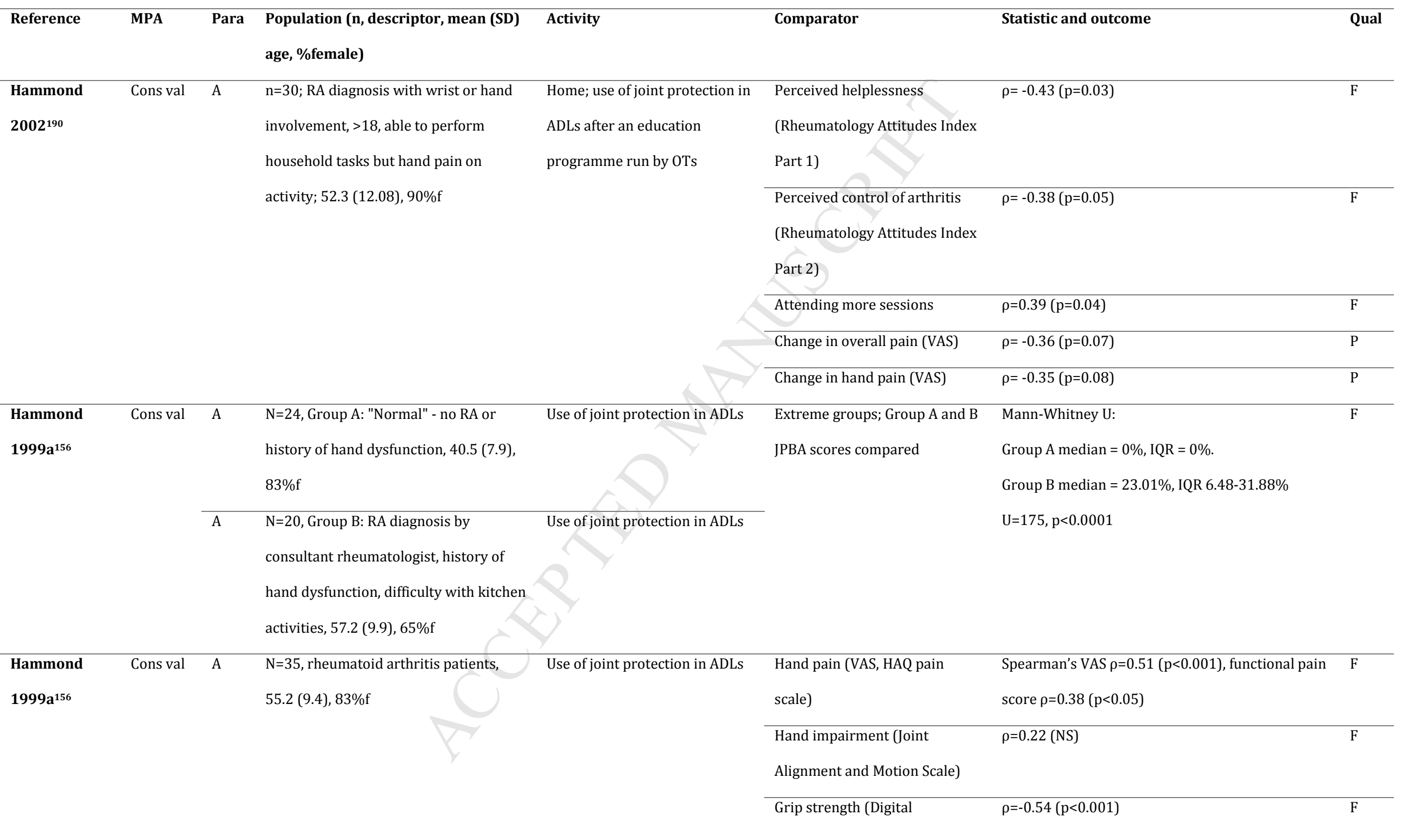




\begin{tabular}{|c|c|c|c|c|c|c|c|}
\hline Reference & MPA & Para & $\begin{array}{l}\text { Population (n, descriptor, mean (SD) } \\
\text { age, \%female) }\end{array}$ & Activity & Comparator & Statistic and outcome & Qual \\
\hline & & & & & Dynamometer) & & \\
\hline & & & & & $\begin{array}{l}\text { Number of painful joints (ACR } \\
\text { criteria) }\end{array}$ & $\rho=0.41(p<0.01)$ & $\mathrm{F}$ \\
\hline & & & & & Functional disability (HAQ) & $\rho=0.33(\mathrm{p}<0.05)$ & $\mathrm{F}$ \\
\hline $\begin{array}{l}\text { Hammond } \\
1999 a^{156}\end{array}$ & FCV & A & $\begin{array}{l}\text { Face validity: NR } \\
\text { Content validity: } n=7 \text { experienced } \\
\text { rheumatology OTs }\end{array}$ & Use of joint protection in ADLs & $\begin{array}{l}\text { Face validity: } 20 \text { JPBA tasks } \\
\text { rated according to whether } \\
\text { they involved the } 5 \text { joint } \\
\text { principles. } \\
\text { Content validity: } 124 \text { codes of } \\
\text { behaviour definitions (normal, } \\
\text { joint protection and functional } \\
\text { adaptations) were developed } \\
\text { from literature and video } \\
\text { observations of RA. Seven } \\
\text { rheumatology OTs reviewed } \\
\text { each behaviour code and scored } \\
\text { it as correct, partially correct or } \\
\text { incorrect. }\end{array}$ & $\begin{array}{l}\text { Each task was rated as being appropriate for } \\
\text { assessing } 2-5 \text { joint protection principles } \\
\text { Kappa: } \kappa=0.6 \text { overall (range for individual tasks } \\
0.46-1.00 \text { (all p<0.01)). } 41.13 \%(51 / 124) \text { codes } \\
\text { had total agreement. }\end{array}$ & $\mathrm{F}$ \\
\hline $\begin{array}{l}\text { Klompenhou } \\
\text { wer } 2000^{159}\end{array}$ & $\begin{array}{l}\text { Resp to } \\
\text { change }\end{array}$ & A & $\begin{array}{l}\mathrm{N}=6 \text { participants: healthy adults } \\
\text { (junior OT students), age NR, gender NR }\end{array}$ & $\begin{array}{l}\text { Lab; } 3 \times 3 \text { groups of observers } \\
\text { each rated a videotape of } 6\end{array}$ & $\begin{array}{l}\text { JPBA tasks performed with 1) } \\
\text { no joint protection knowledge, }\end{array}$ & $\begin{array}{l}\text { Mean scores } \\
\text { 1) } 0.06\end{array}$ & $\mathrm{P}$ \\
\hline
\end{tabular}




\begin{tabular}{|c|c|c|c|c|c|c|c|}
\hline Reference & MPA & Para & $\begin{array}{l}\text { Population (n, descriptor, mean (SD) } \\
\text { age, \%female) }\end{array}$ & Activity & Comparator & Statistic and outcome & Qual \\
\hline & & & $\begin{array}{l}\mathrm{N}=9 \text { observers (different junior OT } \\
\text { students), age NR, gender NR }\end{array}$ & $\begin{array}{l}\text { unique performances and } 2 \\
\text { duplicates of JPBA } \\
\text { performances }\end{array}$ & $\begin{array}{l}\text { 2) after } 1 \mathrm{hr} \text { joint protection } \\
\text { instructions and } 3 \text { ) with verbal } \\
\text { guidance. Unique rating scores } \\
\text { compared at each manipulated } \\
\text { level. }>0.20 \text { considered } \\
\text { clinically significant difference. }\end{array}$ & $\begin{array}{l}\text { 2) } 0.38 \\
\text { 3) } 0.82 \\
\text { All }>0.20 \text { difference }\end{array}$ & \\
\hline $\begin{array}{l}\text { Hammond } \\
1999 a^{156}\end{array}$ & TRR & A & $\begin{array}{l}\mathrm{N}=20 \text {, Rheumatoid arthritis diagnosis, } \\
\text { history of hand dysfunction, difficulty } \\
\text { with kitchen activities, } 57.2(9.9), 65 \% \mathrm{f}\end{array}$ & Use of joint protection in ADLs & Time interval: approx. 8 weeks & Spearman's $\rho=0.91 \mathrm{p}<0.0001$ & $\mathrm{P}$ \\
\hline $\begin{array}{l}\text { Hammond } \\
1999 a^{156}\end{array}$ & $\begin{array}{l}\text { Inter- } \\
\text { rater rel }\end{array}$ & A & $\begin{array}{l}4 \text { OTs with no recent rheumatology } \\
\text { experience, } 1 \text { researcher, age and } \\
\text { gender of OTs and sample NR }\end{array}$ & Use of joint protection in ADLs & $\begin{array}{l}10 \text { videotaped JPBAs of people } \\
\text { with RA were scored by each } \\
\text { OT with regular consultation of } \\
\text { the manual }\end{array}$ & 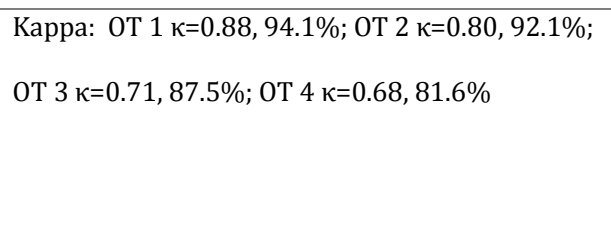 & $\mathrm{P}$ \\
\hline $\begin{array}{l}\text { Klompenhou } \\
\text { wer } 2000^{159}\end{array}$ & $\begin{array}{l}\text { Intra- } \\
\text { rater rel }\end{array}$ & A & $\begin{array}{l}\mathrm{N}=6 \text { participants: healthy adults } \\
\text { (junior OT students), age NR, gender NR } \\
\mathrm{N}=9 \text { observers (different junior OT } \\
\text { students), age NR, gender NR }\end{array}$ & $\begin{array}{l}\text { Lab; JPBA tasks performed with } \\
\text { 1) no joint protection } \\
\text { knowledge, 2) after } 1 \mathrm{hr} \text { joint } \\
\text { protection instructions and 3) } \\
\text { with verbal guidance }\end{array}$ & $\begin{array}{l}3 \times 3 \text { groups of observers each } \\
\text { rated a videotape of } 6 \text { unique } \\
\text { performances and } 2 \text { duplicates; } \\
\text { duplicates assessed }\end{array}$ & ICC $=0.97(0.92-0.99)$ & $\mathrm{P}$ \\
\hline $\begin{array}{l}\text { Klompenhou } \\
\text { wer } \mathbf{2 0 0 0}^{159}\end{array}$ & $\begin{array}{l}\text { Inter- } \\
\text { rater rel }\end{array}$ & A & $\begin{array}{l}\mathrm{N}=6 \text { participants: healthy adults } \\
\text { (junior OT students), age NR, gender NR } \\
\mathrm{N}=9 \text { observers (different junior OT }\end{array}$ & $\begin{array}{l}\text { Lab; JPBA tasks performed with } \\
\text { 1) no joint protection } \\
\text { knowledge, 2) after } 1 \mathrm{hr} \text { joint }\end{array}$ & $\begin{array}{l}\text { 3x3 groups of observers each } \\
\text { rated a videotape of } 6 \text { unique } \\
\text { performances and } 2 \text { duplicates; }\end{array}$ & ICC $=0.93(0.83-0.97)$ & $\mathrm{P}$ \\
\hline
\end{tabular}




\begin{tabular}{|c|c|c|c|c|c|c|c|}
\hline Reference & MPA & Para & $\begin{array}{l}\text { Population (n, descriptor, mean (SD) } \\
\text { age, \%female) }\end{array}$ & Activity & Comparator & Statistic and outcome & Qual \\
\hline & & & students), age NR, gender NR & $\begin{array}{l}\text { protection instructions and 3) } \\
\text { with verbal guidance }\end{array}$ & unique performances assessed & & \\
\hline $\begin{array}{l}\text { Klompenhou } \\
\text { wer } 2000^{159}\end{array}$ & Int cons & A & $\begin{array}{l}\mathrm{N}=6 \text { participants: healthy adults } \\
\text { (junior OT students), age NR, gender NR } \\
\mathrm{N}=9 \text { observers (different junior OT } \\
\text { students), age NR, gender NR }\end{array}$ & $\begin{array}{l}\text { Lab; JPBA tasks performed with } \\
\text { 1) no joint protection } \\
\text { knowledge, 2) after } 1 \mathrm{hr} \text { joint } \\
\text { protection instructions and 3) } \\
\text { with verbal guidance }\end{array}$ & $\begin{array}{l}3 \times 3 \text { groups of observers each } \\
\text { rated a videotape of } 6 \text { unique } \\
\text { performances and } 2 \text { duplicates; } \\
\text { unique rating scores divided } \\
\text { into S-JPBA and A-JPBA }\end{array}$ & Cronbach's alpha $=0.95$ & $P$ \\
\hline
\end{tabular}

Joint Protection Behaviour Assessment acceptability studies (N=1)

Using joint protection strategies Performance rates

in ADLs for $48 \mathrm{mo}$ after a
standard arthritis education

experiencing hand pain on activity;

mean age for control group: 51 years

range: (45-59.25); mean age for joint

protection programme: 52 years range:

(44-59), 76\%f

Polar A1 series HRMs acceptability studies (n=1)

\begin{tabular}{llll}
\hline Segerstahl & Acc & F, D, & $\mathrm{n}=30$, healthy adults sampled on \\
& I & exercise background and motivation, \\
2011 $^{\mathbf{1 6 1}}$ & & $30.0(6.3), 50 \% \mathrm{f}$
\end{tabular}

programme, including $2.5 \mathrm{hrs}$ of

joint protection
83/127 agreed to be recorded performing JPBA

(44/49 at 48mo in intervention, 39/58 in control)

Unclear if others were assessed but not videoed.

High refusal in standard group may relate to non-

intervention

$\begin{array}{lll}\text { Structured and non-structured } & \text { Wear rates, experiences of } & \text { HRM used in 291/383 (76.0\%) of sessions } \\ \text { exercise, including swimming, } & \text { using the HRM } & \text { reported in a diary. 28/30 (93.0\%) chose to use it } \\ \text { running, cycling, strength } & & \text { regularly. } 92(24.0 \%) \text { reported sessions were } \\ \text { training, climbing, horseback } & \text { carried out without the HRM }\end{array}$

training, climbing, horseback

carried out without the HRM

riding, walking, soccer, 
age, \%female)

basketball and gardening, over

3 weeks
Semi-structured interviews and diaries:

Common reasons for non-use were:

inconvenience/awkwardness associated with the chest strap, perceived unsuitability of heart rate monitoring for specific sports such as rock

climbing or windsurfing, lack of time or forgetting to bring it along when exercising

Benefits to HRM: monitors helped understand

cause and effect in exercise behaviour, challenge

or validate subjective feelings, optimise

performance, highlight training patterns, was motivational and fun and offered a sense of accomplishment.

Limitations: lack of surety about the appropriateness of the monitor's guidance and whether it was specific enough, further detail needed in manuals about target behaviours, unsuitability for certain situations, data 
age, \%female)

incompleteness and privacy concerns.

Participants were highly motivated, young, fit, healthy and computer literate. $66.7 \%$ had prior

experience of using a HRM.

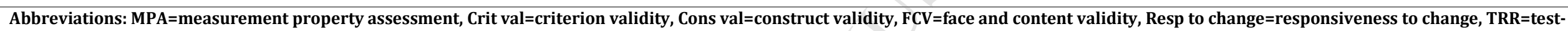

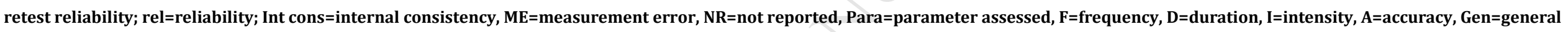
adherence, $\mathrm{O}=$ =ther, $\mathrm{n}=$ number of participants, $\mathrm{SD}=$ standard deviation, $\mathrm{G} 1=$ group 1, \%f=percentage female, $\mathrm{Qual}=\mathrm{COSMIN}$ quality rating (F=Fair, $\mathrm{P}=\mathrm{Poor}$ ), ICC=intra-class correlation coefficient, LOA=limits of agreement, SAM=StepWatch Activity Monitor, PETS=Problematic Experiences of Therapy Scale, JPBA=Joint Protection Behaviour Assessment, RPE=rating of perceived exertion,

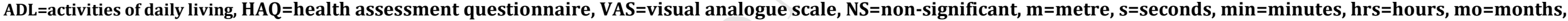
mph=miles per hour, $\mathrm{RCT}=$ randomised controlled trial, $1 \mathrm{RM}=1$ repetition maximum 
Supplementary File 1: List of search terms

\section{Phase 1 Search}

CENTRAL, EED and HTA (2000-April 2013)

(Title, abstract, keywords: "patient compliance" OR Title, abstract, keywords: compliance

OR Title, abstract, keywords: adherence) AND (All text: "rehabilitation" OR All text: rehabilitation)

\section{Phase 2 Searches (Medline example)}

Search strategies were adapted with headings relevant to each database.

\section{Publication type}

1. Validation studies

\section{MeSh}

1. Reproducibility of results

2. Psychometrics

3. Observer variation

4. Discriminant analysis

\section{$\mathbf{T i}+\mathbf{a b}$}

5. Reproducib*

6. Psychometr* 
7. Clinimetri*

8. Clinometr*

9. Observer variation

10. Reliab*

11. Valid*

12. Coefficient

13. "internal consistency"

14. (Cronbach* AND (alpha OR alphas))

15. "item correlation"

16. "item correlations"

17. "item selection"

18. "item selections"

19. "item reduction"

20. "item reductions"

21. Test-retest

22. (test AND retest)

23. (reliab* AND (test OR retest))

24. Stability

25. Interrater

26. Inter-rater

27. Intrarater

28. Intra-rater

29. Intertester

30. Inter-tester

31. Intratester

32. Intra-tester

33. Interobserver

34. Inter-observer 
35. Intraobserver

36. Intra-observer

37. Intertechnician

38. Inter-technician

39. Intratechnician

40. Intra-technician

41. Interexaminer

42. Inter-examiner

43. Intraexaminer

44. Intra-examiner

45. Interassay

46. Inter-assay

47. Intraassay

48. Intra-assay

49. Interindividual

50. Inter-individual

51. Intraindividual

52. Intra-individual

53. Interparticipant

54. Inter-participant

55. Intraparticipant

56. Intra-participant

57. Kappa

58. Kappa's

59. Kappas

60. "coefficient of variation"

61. Generaliza*

62. Generalisa* 
63. Concordance

64. (intraclass AND correlation*)

65. Discriminative

66. "known group"

67. "Factor analysis"

68. "Factor analyses"

69. "factor structure"

70. "factor structures"

71. Dimensionality

72. Subscale*

73. "multitrait scaling analysis"

74. "multitrait scaling analyses"

75. "Item discriminant"

76. "Interscale correlation"

77. "Interscale correlations"

78. ((Error OR errors) AND (measure* OR correlat* OR evaluat* OR accuracy* OR accurate OR precision OR mean))

79. "individual variability"

80. "interval variability"

81. "rate variability"

82. "variability analysis"

83. (uncertainty AND (measurement OR measuring))

84. "standard error of measurement"

85. Sensitiv*

86. Responsive*

87. (limit AND detection)

88. "minimum detectable concentration"

89. Interpretab* 
90. (small* AND (real OR detectable) AND (change OR difference))

91. "Meaningful change"

92. "minimal important change"

93. "minimal important difference"

94. "minimally important change"

95. "minimally important difference"

96. "minimal detectable change"

97. "minimal detectable difference"

98. "minimally detectable change"

99. "minimally detectable difference"

100. "minimal real change"

101. "minimal real difference"

102. "minimally real change"

103. "minimally real difference"

104. "ceiling effect"

105. "floor effect"

106. "item response model"

107. IRT

108. Rasch

109. "Differential item functioning"

110. DIF

111. "computer adaptive testing"

112. "Item bank"

113. "cross-cultural equivalence"

114. qualitative

115. interpret*

116. rating*

117. attach* 
118. meaning*

119. impact*

120. burden

121. feasib*

122. "missing data"

123. "missing values"

124. "data loss"

125. (response OR non-response OR nonresponse)

126. "refusal rate"

127. understand*

128. completion

129. comprehens*

130. wear

131. non-wear

132. nonwear

133. comfort*

134. discomfort

135. eas*

136. appearance

137. safe*

138. (location OR placement)

139. size

140. conceal*

141. usab*

142. utility

143. satisf*

144. accepta*

145. willing* 
146. ability

147. benefit

148. performance

149. obtrusive*

150. pilot*

151. workload

\section{Text word (TX)}

1. Agreement

2. Precision

3. Imprecision

4. "precise values"

5. Repeatab*

6. ((replica* OR repeated) AND (measure OR measures OR findings OR result OR results OR test OR tests))

All the above terms were searched using OR, and the exclusion filter was applied using NOT.

\section{Exclusion filter (All terms combined using OR)}

Publication type

1. "addresses"

2. "biography"

3. "case reports"

4. "comment"

5. "directory"

6. "editorial"

7. "festschrift" 
8. "interview"

9. "lectures"

10. "legal cases"

11. "legislation"

12. "letter"

13. "news"

14. "newspaper article"

15. "patient education handout"

16. "popular works"

17. "congresses"

18. "consensus development conference"

19. "consensus development conference, nih"

20. "practice guideline"

\section{$\mathrm{MeSH}$}

21. NOT (“animals" NOT "humans")

\section{Measure search terms}

\begin{tabular}{|l|l|}
\hline Problematic & TI, AB "problematic experiences of therapy scale" \\
experiences of & \\
therapy scale & \\
\hline StepWatch Activity & $\begin{array}{l}\text { TI, AB “step activity monitor" OR stepwatch OR (monitor AND orthocare) OR } \\
\text { (monitor AND cyma) OR (monitor AND modus) OR (SAM AND monitor* AND } \\
\text { step) }\end{array}$ \\
\hline
\end{tabular}




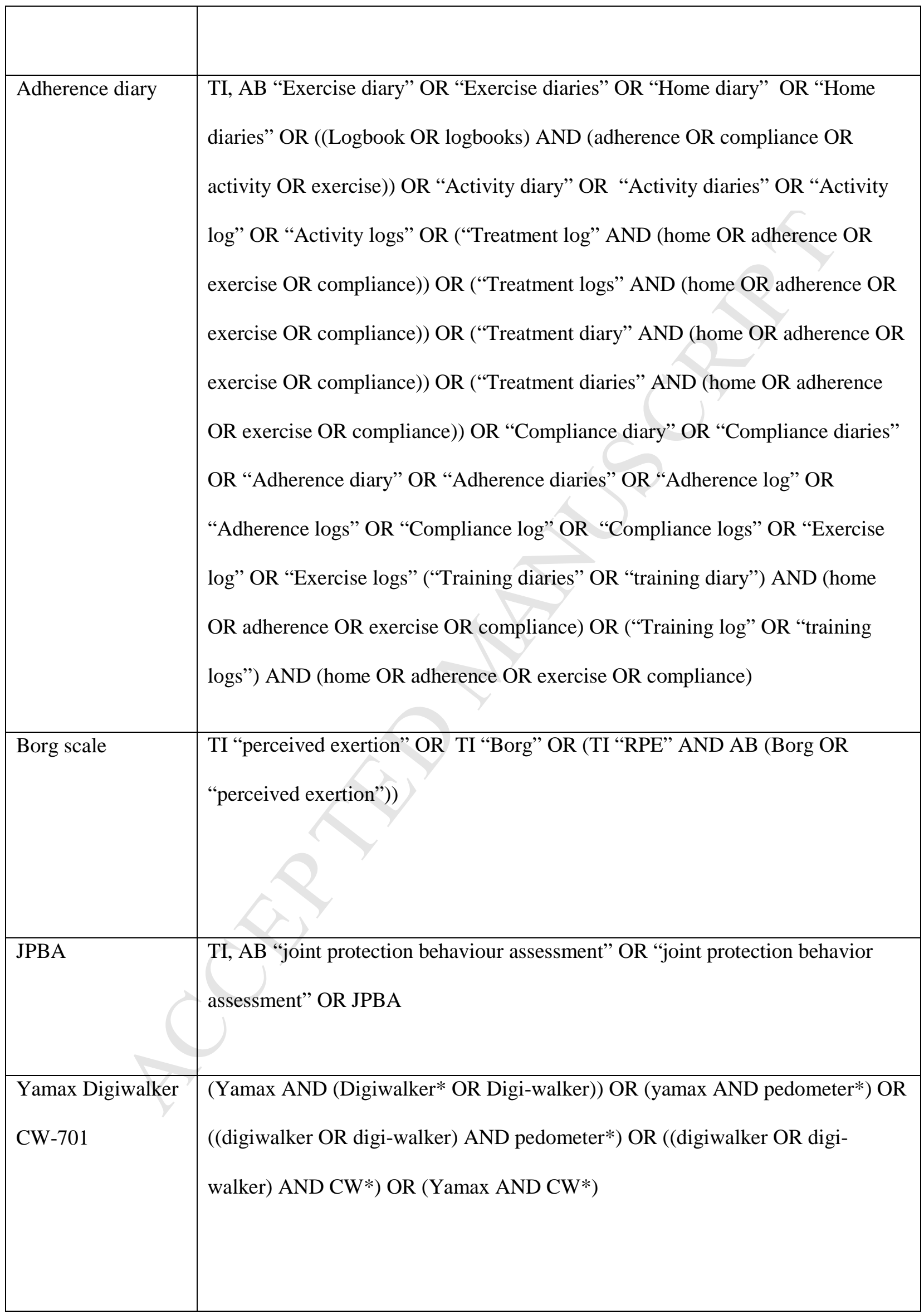




\begin{tabular}{|l|l|}
\hline Polar A1 \& FS1 & TI, AB (Polar AND heart AND monitor*) \\
heart rate monitors & \\
& \\
\hline
\end{tabular}

